\title{
Deformation behavior upon two- step loading in a magnesium alloy sheet
}

\section{$\operatorname{AUTHOR}(\mathrm{S})$ :}

Hama, Takayuki; Tanaka, Yuhki; Uratani, Masato; Takuda, Hirohiko

\section{CITATION:}

Hama, Takayuki ...[et al]. Deformation behavior upon two-step loading in a magnesium alloy sheet. International Journal of Plasticity 2016, 82: 283-304

\section{ISSUE DATE:}

2016-07

URL:

http://hdl.handle.net/2433/218302

\section{RIGHT:}

(c) 2016. This manuscript version is made available under the CC-BY-NC-ND 4.0 license

http://creativecommons.org/licenses/by-nc-nd/4.0/; The full-text file will be made open to the public on 01 July 2018 in accordance with publisher's 'Terms and Conditions for Self-Archiving'.; This is not the published version. Please cite only the published version.; この論文は出版社版でありません。引用の際には出版社版をご確認ご利用ください。 
Deformation behavior upon two-step loading in a magnesium alloy sheet

\author{
Takayuki HAMA* \\ Yuhki TANAKA \\ Masato URATANI \\ Hirohiko TAKUDA \\ Graduate school of Energy Science, Kyoto University, Yoshida-honmachi, Sakyo-ku, \\ Kyoto 606-8501, Japan
}

Corresponding author: Takayuki HAMA(hama@energy.kyoto-u.ac.jp)

Highlights

- A two-step loading test was conducted on a magnesium alloy sheet.

- Strong anisotropic deformation occurred when precompression was applied.

- The anisotropic deformation was predicted well using a crystal-plasticity FEM.

- The anisotropic deformation resulted from detwinning activity.

- The role of twinned region on macroscopic deformation was discussed numerically. 


\section{Abstract}

Two-step loading tests were performed on an AZ31 rolled magnesium alloy sheet with strong basal texture in the normal direction, and the deformation behavior such as a stress-strain curve, Lankford value, and texture evolution was investigated both experimentally and numerically. When the sheet was subjected to compression in the rolling direction followed by tension in different directions, the following characteristic deformation was observed during the second loading: The sigmoidal shape of the stress-strain curve was more pronounced when the sheet was stretched in the rolling direction but less pronounced as the loading direction approached the transverse direction. The Lankford value during the second loading was much smaller than that of the virgin sheet. Observation of the microstructure showed that the detwinning activity during the second loading decreased as the loading direction approached the transverse direction. The aforementioned deformation behavior was qualitatively well predicted using a crystal-plasticity finite-element method. The crystal-plasticity analysis was then used to investigate the underlying deformation mechanism upon two-step loading, focusing especially on the effect of twinning and detwinning activities.

Keywords: B. Constitutive behavior; B. Crystal plasticity; Strain-path change; A. twinning; A. Microstructures 


\section{Introduction}

Magnesium (Mg) alloy sheets are expected as possible lightweight materials for thin-walled structural components [Kulekci, 2008; Easton, et al., 2008; Taub and Luo, 2015; Frankel, 2015], but their use is still low [Askari et al., 2014; Suh et al., 2015]. One of the reasons that impede application of $\mathrm{Mg}$ alloy sheets is their deformation characteristics at room temperature, such as strain-path dependency [Hama et al., 2014, 2015], tension-compression asymmetry [Lou et al., 2007; Hama et al., 2012; Nguyen et al., 2014; Kurukuri et al., 2014], anisotropic work hardening [Hama et al., 2012a; Steglich et al., 2012], and nonlinear response during unloading [Hama and Takuda, 2011; Hama et al., 2013; Lee and Gharghouri, 2013,Wang et al., 2013a, Hama et al, 2015a]. However, the details of these mechanisms are still open to discussion.

The strong characteristic deformation behavior of $\mathrm{Mg}$ alloys results primarily from the hexagonal close-packed (hcp) structure with significantly different critical resolved shear stresses depending on the slip and twinning systems. In particular, easy activation of direction-dependent deformation twinning affects significantly the mechanical behavior [Jiang et al., 2007; Knezevic et al., 2010; Fernández et al., 2013; Balík et al., 2016; Guo et al., 2016]. Moreover, a strong basal texture generally develops in rolled sheets [Lou et al., 2007]; thus, the anisotropy in deformation behavior is pronounced [Hama et al., 2014]. Therefore, the deformation characteristics in rolled Mg alloy sheets are being actively studied to promote more effective use of $\mathrm{Mg}$ alloy sheets [Ghaffari Tari et al., 2014; Steglich et al., 2014]. Thanks to recent advances in the modeling of twinning and detwinning, crystal-plasticity models are being widely utilized to study the macroscopic and mesoscopic deformation characteristics of $\mathrm{Mg}$ alloys [Proust et al., 2009; Hama and Takuda, 2011; Hama and Takuda, 2012b; Oppedal et al., 2013; Wang et al., 2013a, 2013b; Hama et al., 2013a, 2013b; Liu and Wei, 2014; Wu et al., 2014; Li et al., 2014; Gu et al., 2014; Cheng and Ghosh, 2015; Qiao et al., 2015; Sánchez-Martín et al., 2015; Abdolvand et al, 2015; Dogan et al., 2015; Kabirian et al., 2015; Mayama et al., 2015].

Because sheet metals experience in general strain-path changes during forming processes, the work-hardening behavior under such changes is an important deformation characteristic. Two-step deformation is a representative deformation mode involving strain-path changes; thus, it has been studied extensively in various sheet metals. One of the typical examples of two-step loading is reverse loading, where the angle between the first and second loading axes, $\theta$, is zero but the loading directions are opposite. Strong strain-path dependency occurs when Mg alloy sheets are subjected to reverse loading, as mentioned earlier. A sigmoidal curve occurs under tension following compression, 
whereas such a curve does not occur under compression following tension [Lou et al., 2007; Hama et al., 2012; Nguyen et al., 2014; Kurukuri et al., 2014; Hama et al., 2015b]. Recently, it was reported that this strong strain-path dependency occurs not only in rolled sheets with strong basal textures but also in cast sheets with random crystallographic orientations [Hama et al., 2015b]. The underlying mechanisms of this strain-path dependency have been studied extensively, in particular with respect to the effect of twinning and detwinning [Lou et al., 2007; Proust et al., 2009; Hama et al., 2012; Hama and Takuda, 2012b; Wang et al., 2013b; Wu et al., 2014; Nguyen et al., 2014; Kurukuri et al., 2014; Qiao et al., 2015], and it is now understood that one of the mechanisms is the activities of twinning and detwinning.

Concerning cases with $\theta \neq 0^{\circ}$, the work-hardening behavior has been studied widely in face- and body-centered cubic metals such as steel and aluminum alloy sheets both experimentally and numerically [Kim and Yin, 1997; Teodosiu and Hu, 1998; Hoc and Forest, 2001; Peeters et al., 2001; Hahm and Kim, 2008; Holmedal et al., 2008; Rauch et al., 2011; Wang et al., 2012; Segurado et al., 2012; Gérard et al., 2013; Kitayama et al., 2013; Barlat et al., 2013; Mánik et al., 2015]. For Mg alloys, on the other hand, a few studies on two-step compression tests have recently been reported. Sarker and Chen (2014) conducted a test of precompression in the extrusion direction (ED), followed by compression in the normal direction (ND) in an extruded AM30 Mg alloy with a strong basal texture in the ND, and they investigated the work-hardening behavior such as yield strength, ultimate compressive strength and the twinning activities, including secondary twins and detwinning. Xu et al. (2014) studied the generation of $\{10 \overline{1} 2\}-\{10 \overline{1} 2\}$ double twins and their effect on work-hardening behavior under precompression in the rolling direction (RD), followed by compression in the transverse direction (TD) in a hot-rolled AZ31 Mg alloy sheet with a strong basal texture in the ND. Shi et al. (2015) also examined the generation and role of $\{10 \overline{1} 2\}-$ $\{10 \overline{1} 2\}$ double twins under precompression in the $\mathrm{RD}$, followed by compression in the TD in a hot-rolled commercial AZ31 alloy sheet with a strong basal texture in the ND. It is evident from the abovementioned examples that most of the previous studies for $\mathrm{Mg}$ alloys have focused primarily on twinning activity and its effect on work-hardening behavior [Sarker et al., 2014; Xin et al., 2014; Xin et al., 2015; Park et al., 2015].

The deformation behavior during the second loading in two-step compression would be governed by both twinning and detwinning, as described in the abovementioned studies. On the other hand, it is expected that the deformation behavior during the second loading may be governed primarily by detwinning when Mg alloy sheets are subjected to two-step compression-tension, i.e., compression in the first 
loading, followed by tension along different directions in the second loading. Therefore, the deformation behavior would be notably different between two-step compression and two-step compression-tension. However, in contrast to two-step compression, the deformation behavior under two-step compression-tension has hardly been investigated. To the best of our knowledge, Park et al. (2013a) first studied the deformation characteristics of a hot-rolled AZ31 Mg alloy plate with a strong basal texture in the ND under two-step compression-tension. They investigated experimentally the deformation behavior under compression in the RD followed by tension or compression in the RD, the $\mathrm{TD}$, and at $45^{\circ}$ from the $\mathrm{RD}$, and they found that the detwinning activity decreased as the second loading direction approached the TD. Moreover, they presumed that slip and twinning activities in the twinned region during the second loading would yield pronounced anisotropic deformation behavior. Because their study focused on twinning activity, a further effort is required to understand macroscopic deformation behavior such as yield stress and work hardening. Moreover, rectangular-shaped specimens were utilized in their study; thus, changes to the Lankford value, which is an important measure in sheet metal forming, upon the two-step loading were not investigated. Because twinning and detwinning activities affect the thickness strain in rolled $\mathrm{Mg}$ alloy sheets with strong basal texture, it is expected that the Lankford value would change significantly upon two-step loading. However, such results have not been reported yet.

It should also be mentioned that, unlike cubic metals, a theoretical model of the deformation behavior under two-step loading in $\mathrm{Mg}$ alloys has scarcely been studied except for reverse loading. Very recently, Wen et al. (2015) conducted two-step tension tests on a rolled $\mathrm{Mg}$ alloy sheet with a strong basal texture in the ND and investigated the mechanical behavior, including the stress-strain curve and evolution of the Lankford value. A dislocation density-based crystal-plasticity model was also used to discuss the deformation mechanism. Note that the twinning activity was negligible in their tests, and detwinning was in fact neglected in their model. As far as we know, simulation of the deformation behavior upon two-step loading with $\theta \neq 0^{\circ}$, where the activities of twinning and detwinning are significant, has not been reported.

In the present study, the deformation characteristics of a rolled AZ31 Mg alloy sheet under two-step loading was investigated both experimentally and numerically. The sheet was subjected to tension or compression along the RD in the first loading, followed by tension along different directions in the second loading. The mechanical behavior, including stress-strain curves and Lankford values, and the microstructure evolution were studied in detail. A crystal-plasticity finite-element method (FEM) was also used to investigate the underlying deformation mechanism with respect to 
microscopic slip and twinning activities.

\section{Experimental procedures}

A commercially rolled AZ31B Mg alloy sheet (Osaka Fuji Corporation) was used as a material under test. The $\mathrm{Mg}$ alloy sheet was annealed with a condition of $350{ }^{\circ} \mathrm{C}$ for $1.5 \mathrm{~h}$ before it was received. The nominal thickness of the received sheet was $1.0 \mathrm{~mm}$. Table 1 summarizes the mechanical properties of the sample obtained from a uniaxial tensile test along the RD at room temperature.

The experimental procedure of the two-step loading test is described. It is schematically illustrated in Fig. 1. The experiment was conducted at room temperature. Firstly, a large sample with the gauge length of $156 \mathrm{~mm}$ and the gauge width of $60 \mathrm{~mm}$ (Fig. 2 (a)) was subjected to uniaxial tension or compression along the RD. The geometry was determined using FEM, such that sufficiently uniform stress and strain distributions were attained near the center of the gauge section. A nominal strain exerted on the large sample was either $6 \%,-3 \%$, or $-6 \%$. Note that comb-shaped dies [Kuwabara et al., 2011; Hama et al., 2012] were employed to perform the test using the large sample without involving buckling during compression, as schematically illustrated in Fig. 3. The compressive through-thickness stress imposed was less than $1.0 \%$ of the $0.2 \%$ proof stress. The comb-shaped dies and the sample were coated with hydraulic oil before the test to reduce friction.

Subsequently, small samples with the gauge length of $20 \mathrm{~mm}$ and the gauge width of $6 \mathrm{~mm}$ (Fig. 2 (b)) were cut from the center part of the prestrained large sample along different directions by using wire electric discharge machining. The photograph of small sample preparation is displayed in Fig. 4. The angles between the first and second loading axes, $\theta$, were $0,30,45,60$, and $90^{\circ}$. Thereafter, a uniaxial tensile test was conducted using the small samples to a nominal strain of $10 \%$. Note that the stressstrain curves obtained in the second loading were almost independent of the position on the prestrained large sample, suggesting that the texture evolution during the first loading would be approximately uniform in the center part of the prestrained large sample.

In the both tests using the large and small samples, an initial strain rate was set to approximately $5.0 \times 10^{-4} / \mathrm{s}^{-1}$. A uniaxial strain gauge (Kyowa Electronic Instruments, KFEM) was attached to the samples to measure nominal strains in the loading direction.

The evolution of crystallographic orientation was measured on a TD-ND plane of the $-6 \%$ prestrained sheet using electron backscatter diffraction (EBSD). The surface was polished by using cross-section polishing. The measurements were conducted with 
a step size of $0.3 \mu \mathrm{m}$. OIM-Analysis 7 (TSL Solutions) was used to analyze the measured data. Note that measured data with a confidence index less than 0.1 were omitted to increase the accuracy of the analyses. Because reliable EBSD measurement of prestrained samples was difficult due to high dislocation densities, the samples were annealed for $12 \mathrm{~h}$ at $160{ }^{\circ} \mathrm{C}$ before the measurement to remove the dislocations. This annealing condition was employed because it had been reported in the literature [Xin et al., 2014] that the annealing did not damage twin structures.

The area fraction of twins was used to examine twinning and detwinning activities quantitatively. The area fraction, $R_{t w i n}$, is defined as

$$
R_{\text {twin }}=\frac{A_{\text {twin }}}{A} \times 100 \text {, }
$$

where $A$ and $A_{\text {twin }}$ denote respectively the entire area of the observed microstructures and the area of twins that are present. At least five different photographs of the microstructures were measured for each condition. For the sake of convenience, microstructures observed using an optical microscope were used for the measurement of area fraction. For the microstructure observation using an optical microscope, a TD-ND plane was mechanically polished and then chemically etched with a mixture of picric acid, ethanol, acetic acid, and distilled water, as in our previous study [Hama et al., 2012, 2014b].

\section{Crystal-plasticity FEM}

\subsection{Constitutive equation}

Because the formulations have been explained in detail elsewhere [Hama and Takuda, 2011, 2012b; Hama et al., 2013], a description is given briefly. The reader is referred to the literatures for details.

The viscoplastic power law was used to represent the slip rate of the $\alpha$-slip system, $\dot{\gamma}^{(\alpha)}$, as follows [Pan and Rice, 1983] :

$$
\frac{\dot{\gamma}^{(\alpha)}}{\dot{\gamma}_{0}}=\left|\frac{\tau^{(\alpha)}}{\tau_{\mathrm{Y}}^{(\alpha)}}\right|^{\frac{1}{m}} \operatorname{sign}\left(\tau^{(\alpha)}\right),
$$

where $\dot{\gamma}_{0}$ denotes the reference-strain rate, $m$ denotes the rate-sensitivity exponent, and $\tau^{(\alpha)}$ denotes the resolved shear stress. $\tau_{\mathrm{Y}}^{(\alpha)}$ denotes the deformation resistance of slip system and its evolution law is given using the interaction matrix, $q_{\alpha \beta}$, and the hardening rate, $h^{(\beta)}$, in the form:

$$
\dot{\tau}_{\mathrm{Y}}^{(a)}=\sum_{\beta} q_{\alpha \beta} h^{(\beta)}\left|\dot{\gamma}^{(\beta)}\right| .
$$


The following hardening laws are utilized [Graff et al., 2007; Hama and Takuda, 2011]:

$$
h^{(\alpha)}=h_{0}
$$

and

$$
h^{(\alpha)}=h_{0}\left(1-\frac{\tau_{0}}{\tau_{\infty}}\right) \exp \left(-\frac{h_{0} \bar{\gamma}}{\tau_{\infty}}\right),
$$

where $\tau_{0}$ denotes the initial critical resolved shear stress and $h_{0}$ and $\tau_{\infty}$ are the hardening parameters. $\bar{\gamma}$ is given by $\bar{\gamma}=\sum_{\alpha} \int\left|\dot{\gamma}^{(\alpha)}\right| \mathrm{d} t$.

Following past studies [Hama and Takuda, 2012; Wang et al., 2013b], kinematic hardening is not taken into consideration in this study to simplify the problem. On the other hand, recently Wen et al. (2015) reported that in their simulation of two-step tension tests of a rolled Mg alloy sheet back stress exerted some influence on the yield stress in the second loading. Moreover, Qiao et al. (2015) presumed that back stress would affect the work-hardening behavior when a Mg alloy was subjected to cyclic loading. These past studies suggest that implementation of a kinematic hardening model may be required to thoroughly investigate the deformation behavior of $\mathrm{Mg}$ alloys. This will be done in future work.

\subsection{Slip and twinning systems}

The present crystal-plasticity model takes basal $<\mathrm{a}>$, prismatic $<\mathrm{a}>$, and pyramidal $<\mathrm{c}+\mathrm{a}>$ slip systems and $\left\{\begin{array}{llll}1 & 0 & \overline{1} & 2\end{array}\right\}$ tension twinning systems into account. Linear [eq.(4)] and Voce [eq.(5)] hardening laws are respectively utilized for basal slip and nonbasal slip.

\subsection{Twinning and detwinning model}

Thus far several twinning and detwinning models have been proposed [e.g. Proust et al., 2009; Wang et al., 2013b; Gu et al., 2014]. In the present study a twinning and detwinning model proposed by the present authors [Hama and Takuda, 2012; Hama et al., 2013] was used. This model had been developed on the basis of a twinning model proposed by Van Houtte (1978). The present model assumes that twinning is active only when tensile stress acts on the $c$-axis, i.e., $\tau^{(\alpha)}>0$, and detwinning can be activated when the sign of $\tau^{(\alpha)}$ changes from positive to negative. Eqs. (2) and (4) are used to describe the evolution of twinning as well as detwinning. Note that the initial strength of 
detwinning is set to be an independent material parameter that should be determined prior to carrying out the simulation. The slip systems are subjected to the twinning rotation when the volume fraction in a grain, $f^{(\alpha)}=\bar{\gamma}_{\text {twin }}^{(\alpha)} / \gamma_{\text {ref }}$, where $\gamma_{\text {ref }}$ and $\bar{\gamma}_{\text {twin }}^{(\alpha)}$ denote respectively the reference-shear strain and the accumulated shear strain induced by twinning, exceeds a threshold value, $f_{\mathrm{th}}^{(\alpha)}$, which is randomly predetermined to be in the range $0.3 \leq f_{\mathrm{th}}^{(\alpha)} \leq 1.0$. A similar procedure is utilized for the detwinning rotation.

The abovementioned crystal-plasticity model was implemented into a static FEM. The rate tangent modulus method [Pierce et al., 1984] and the generalized $r_{\min }$ strategy were utilized to prevent a rapid rise in the nonequilibrated forces due to the explicit time integration. It is worth noting that the generalized $r_{\text {min }}$ strategy is in fact useful for preventing simulations from being unstable during twinning or detwinning rotation.

One of the advantages of the crystal-plasticity FEM is that elasticity can be easily taken into account in the simulation. Because Mg alloy show large elastic recovery and strong nonlinear deformation during unloading, it is important to evaluate these characteristics when the Lankford value is measured. In the present study, the utilization of the crystal-plasticity FEM enabled a comparison of the Lankford value between the experiment and the simulation as will be described in section 4.2, which is advantageous compared to visco-plastic models [e.g. Proust, et al., 2009].

\subsection{Simulation procedures}

The finite-element model displayed in Fig. 5 was utilized. The model consisted of 10 uniform elements in each direction. A type of finite element was the 8-node isoparametric brick element. The crystallographic orientations of the virgin sheet measured using EBSD analysis were randomly assigned to each integration point of the finite-element model. Note that each finite element had a different initial crystallographic orientation. The pole figures of the virgin sheet are depicted in Fig. 6(a). A basal texture free of twinning appeared in the present Mg alloy sheet, as shown in Fig. 6(b). The average grain size was approximately $14 \mu \mathrm{m}$. As explained in our previous studies [Hama and Takuda, 2011, 2012a, 2012b; Hama et al., 2013], the abovementioned simulation model gave qualitatively reasonable results for the deformation behavior in polycrystalline $\mathrm{Mg}$ alloy sheets, including reverse loading, unloading, and biaxial tension.

The simulation procedure of the two-step loading was as follows. For the first loading, the $x, y$, and $z$ directions were respectively specified to be the RD, TD, and ND, and the finite-element model was stretched or compressed in the $x$ direction by imposing displacement boundary conditions to the plane $x=l$ [Fig. 5]. When a 
predetermined strain was attained, the model was unloaded until the forces became negligibly small. Thereafter, the crystallographic orientations and the residual stress tensors were rotated around the z-axis (ND) with respect to angle $\theta$. At the same time, the geometry of the finite-element model was initialized. Subsequently, for the second loading, the model was stretched until a tensile strain of $10 \%$ was attained. The plane-symmetry conditions were imposed to the planes $x=0, y=0$, and $z=0$ in both the first and second loadings.

\subsection{Parameter identification}

Because elastic anisotropy is not significant in $\mathrm{Mg}$, isotropic elasticity was assumed. Young's modulus, Poisson's ratio, and the rate-sensitivity exponent were respectively taken to be $E=42 \mathrm{GPa}, v=0.3$, and $m=0.02$ [Hama et al., 2013; Hama and Takuda, 2011], which are the typical values for Mg alloys. The reference-strain rate was taken to be $\dot{\gamma}_{0}=0.001 \mathrm{~s}^{-1}$.

The hardening parameters were identified in the following manner. Considering the strong basal texture of rolled Mg alloy sheets, the Lankford value was assumed to be determined primarily by the ratio of activities between prismatic slip and pyramidal $<\mathrm{c}+\mathrm{a}>$ slip. This assumption led to the identification procedure, which consisted of the following four steps. Note that the parameters used in our previous studies [Hama and Takuda, 2011, 2013] were used as an initial guess.

(1) The hardening parameters for basal slip were roughly determined to achieve reasonable agreement in the initial yield stress under tension.

(2) The ratios of the hardening parameters between prismatic slip and pyramidal $<\mathrm{c}+\mathrm{a}>$ slip were roughly estimated to obtain reasonable agreement in the evolution of the Lankford value under tension.

(3) The hardening parameters for prismatic slip and pyramidal $<c+a>$ slip, as well as basal slip, were adjusted to achieve reasonable agreement in the stress-strain curve under tension.

(4) The hardening parameters for twinning and detwinning were determined using the stress-strain curves under compression and compression-tension, respectively.

The above four steps were repeated until the stress-strain curves under tension, compression, and compression-tension and the evolution of the Lankford value were reasonably reproduced using a single set of parameters.

The interaction matrix, $q_{\alpha \beta}$, also plays an important role in the prediction of work-hardening behavior; thus, it is being investigated actively in $\mathrm{Mg}$ both 
experimentally [Hiura and Niewczas, 2015] and numerically [Bertin et al., 2014]. However, an effective model of the latent hardening has not been established yet. In the present study, the parameters of $q_{\alpha \beta}$ reported by Graff et al. (2007) shown in Table 2 were utilized because the deformation behavior under uniaxial tension and compression, unloading, reverse loading, and biaxial tension could be predicted fairly well by utilizing the parameters in our previous study [Hama and Takuda, 2011, 2012; Hama et al., 2013]. Note that presently it is difficult to fully validate this choice only from the results of monotonic and uniaxial loading. For instance, different interaction matrixes were used in the crystal plasticity analyses of the deformation behavior of $\mathrm{Mg}$ alloys [e.g. Wang et al., 2013; Graff et al., 2007; Hama and Takuda, 2012], and the simulation results were in fairly good agreement with experimental results irrespective of the interaction matrixes. Moreover, great care is necessary when the effect of the interaction matrix on the simulation accuracy is discussed because the interaction matrix is strongly related to the hardening parameters in simulation.

Experimental data used for the parameter identification were obtained using a medium-sized sample with a gauge length of $53.5 \mathrm{~mm}$ [Hama et al. 2012]. Comb-shaped dies were utilized in all conditions, i.e., monotonic tension, monotonic compression, and compression-tension, to unify the experimental conditions. The hardening parameters identified using the abovementioned procedure are displayed in Table 3. The reference-shear strain was taken to be $\gamma_{\text {ref }}=0.17$. The stress-strain curves under tension, compression, and compression-tension, and the evolution of the Lankford value under tension in the $\mathrm{RD}$ obtained from the experiment and the simulation are depicted in Fig. 7. Note that the Lankford value was measured after unloading at each strain in both experiment and simulation. The simulation results are denoted by original simulation in Fig. 7. The simulation results are in fairly good agreement with the experimental results, demonstrating that the parameters are acceptable.

\section{Results and discussion}

4.1 Effect of initialization of model geometry on simulation result

As explained before, the geometry of the finite-element model was initialized at the beginning of the second loading. This initialization would affect the prediction accuracy of stress-strain curves, as well as the evolution of the Lankford value. To evaluate its effect on the simulation results, the results of compression-tension and monotonic tension were compared between the original simulation, explained in section 3.5, and the simulation with the initialization (two-step simulation). The 
simulation procedure of the latter case is as follows. For compression-tension, the model is compressed to a strain of $4 \%$ in the $\mathrm{RD}$, followed by initialization of the model and tension in the RD, i.e., $\theta=0^{\circ}$. For monotonic tension, the model is stretched to a strain of $6 \%$ in the RD, followed by the initialization and tension in the RD.

The stress-strain curves obtained from the two-step simulation are presented in Figs. 7(a) and 7(b). In compression-tension, the occurrence of the sigmoidal curve is clearly delayed as compared to that of the original simulation. In monotonic tension, the result is almost identical to the original result. In contrast, regarding the evolution of the Lankford value presented in Fig. 7(c), the results at strains larger than 5\%, i.e., after the initialization of the model, are apparently larger than the original results. These results indicate that, as we expected, the initialization of the model geometry somewhat affects the prediction accuracy of the stress-strain curve and the Lankford value. Therefore, in the following, we focus our attention instead on qualitative trends of the simulation results.

\subsection{Results of mechanical behavior}

In this section, the results obtained in the second loading are described. The experimental and simulation results of true stress-strain curves obtained with different angles are shown in Fig. 8. Note that all the results are shifted to start from the origin. In the virgin sheet [Fig. 8(a)], the strain hardening behavior is almost independent of the angle, whereas the yield and flow stresses are the largest for the angle $\theta=0^{\circ}$ and decrease slightly as the angle increases. These tendencies are predicted qualitatively well in the simulation results. However, at the same time, the flow stress is slightly larger in the simulation results than in the experimental results, irrespective of the angle. This inconsistency is owing to the friction between the sample and the comb-shaped die; the hardening parameters were determined on the basis of the experimental results obtained using the medium sample with the comb-shaped dies [Fig. 7], whereas the comb-shaped dies were not utilized in the second loading of the two-step loading test. Therefore, we have to bear this inconsistency in mind when comparing the stress-strain curves between the experimental and simulation results below.

The difference in the flow stress as a function of the angle is more pronounced in the $6 \%$ prestrained sheet [Fig. 8(b)] than in the virgin sheet. In the $6 \%$ prestrained sheet, the slope of the curve changes sharply upon yielding in the case of the angle $\theta=0^{\circ}$, whereas the change in slope is gentle for other angles. Interestingly, the flow stress in the case of the angle $\theta=90^{\circ}$ is slightly larger than that of the angles $\theta=45$ and $60^{\circ}$ at strains larger than approximately $6 \%$. Similar results were also reported in the literature 
[Wen et al., 2015]. The abovementioned tendencies are predicted qualitatively well in the simulation results.

Strain hardening behavior in the $-3 \%$ and $-6 \%$ prestrained sheets is significantly different from that of the 6\% prestrained sheet, as shown in Figs. 8(c) and 8(d). The evolution of the work-hardening rate is also displayed in Fig. 9. For both $-3 \%$ and $-6 \%$ prestrained sheets, the yield stress rises with rising angle. A sigmoidal curve is presented for the angles $\theta=0$ and $30^{\circ}$, whereas a monotonic increase is presented for the angles $\theta$ $=45,60$, and $90^{\circ}$. In contrast, the flow stress in the latter stage of deformation is rather complicated; the flow stress is the largest for the angle $\theta=90^{\circ}$ and the flow stresses for the angles $\theta=0$ and $30^{\circ}$ are larger than those of the angles $\theta=45$ and $60^{\circ}$. The abovementioned tendencies are irrespective of the amount of compressive prestrain and are well predicted in the simulation results for the $-6 \%$ prestrained sheet. In the $-3 \%$ prestrained sheet, the strain hardening behavior and the yield stresses are predicted fairly well in the simulation. On the other hand, the flow stresses in the latter stage for the angles $\theta=0,30$, and $45^{\circ}$ are overestimated in the simulation; thus, the flow stresses are larger for the angles $\theta=0$ and $30^{\circ}$ than for the angle $\theta=90^{\circ}$. Apparently, this magnitude relationship of the flow stress is different from that of the experiment. Note that in the experimental results of the $-3 \%$ prestrained sheet the slope of the stressstrain curve changes sharply at the very beginning for the angles $\theta=0,30$, and $45^{\circ}$ as designated by $\mathrm{A}$ in Fig. 8(c). This sharp change in the slope can also be observed in the work-hardening rate as designated by A in Fig. 9(a). In contrast, this trend does not occur in the simulation results. Because this trend does not occur in the conventional compression-tension as shown in Fig. 7(b), this sharp change in the slope would result from the preparation of the small sample.

Subsequently, the evolution of the Lankford value is described. The Lankford value was measured after unloading at each strain in both experiment and simulation. Because it is presumed from the stress-strain curves [Figs. 8(c) and 9(a)] that for the $3 \%$ prestrained sheet a reliable comparison between the experiment and the simulation cannot be performed in the evolution of the Lankford value, the results of the virgin, $6 \%$ prestrained, and $-6 \%$ prestrained sheets are explained below.

Fig. 10 displays the experimental and simulation results of the Lankford values measured after the second loading, i.e., at a nominal strain of $10 \%$. The results for the virgin sheet using the small sample are also provided. In the experimental results, the Lankford values for the $6 \%$ prestrained sheet are close to those of the virgin sheet, indicating that the Lankford value is insensitive to pre-tension. On the other hand, the Lankford values are much smaller in the $-6 \%$ prestrained sheet than in the other two 
sheets, regardless of the angle. This result shows that thickness strain occurs much more easily in the $-6 \%$ prestrained sheet than in the other two sheets.

The simulations tend to either underestimate or overestimate the Lankford values in all the sheets. On the other hand, the following tendencies observed in the experimental results are predicted qualitatively well in the simulations. For both virgin and $6 \%$ prestrained sheets, the Lankford values increase as the angle increases from $\theta=$ 0 to $45^{\circ}$ and decreases from $\theta=45$ to $90^{\circ}$. For the $-6 \%$ prestrained sheet, the Lankford values are smaller than those of the other sheets, irrespective of the angle, and it is the largest for the angle $\theta=60^{\circ}$.

The aforementioned results suggest that the present simulation model, including the twinning model, hardening parameters, latent hardening moduli, and simulation procedure of the two-step loading, have some room for further improvement in terms of quantitatively accurate predictions. Nevertheless, we consider that the obtained simulation results are acceptable because the results can capture the qualitative trends observed in the experimental results, at least for the virgin, 6\% prestrained, and $6 \%$ prestrained sheets.

\subsection{Microstructures}

Figs. 11 and 12 show the results of EBSD measurements for the $-6 \%$ prestrained sheet. The microstructures were measured at the beginning and end of second loading, i.e., at nominal strains of 0 and $10 \%$. Note that almost all of the activated twins observed in our measurements were $\left\{\begin{array}{llll}1 & 0 & \overline{1} & 2\end{array}\right\}$ tension twinning, i.e., $\left\{\begin{array}{lllll}1 & 0 & \overline{1} & 1\end{array}\right\}$ compression twinning was hardly observed. Therefore, in the following, the term “twinning” denotes $\left\{\begin{array}{lllll}1 & 0 & \overline{1} & 2\end{array}\right\}$ tension twinning unless otherwise noted.

Strong peaks appear in the RD in the (0001) pole figure at the beginning of the second loading [Fig. 11(a)] because of the twinning activity [Fig. 12(a)]. On the other hand, in the $\langle 10 \overline{1} 0>$ pole figure, the intensity is relatively stronger in the TD than in the $\mathrm{RD}$. The textures at the end of the second loading are significantly different, depending on the angle. For the angle $\theta=0^{\circ}$, a twinned region is hardly visible [Fig. 12(b)] and the pole figures [Fig. 11(b)] are close to the initial ones [Fig. 6(a)], which indicates that detwinning is very active. As the angle increases, the remaining twinned region increases [Figs. 12(c)-12(f)] and the peaks in the RD in the (0001) pole figure and in the TD in the $\langle 10 \overline{1} 0>$ pole figure become more pronounced [Figs. 11(c)-11(f)]. These results show that the detwinning activity decreases as the angle changes from $\theta=0$ to 
$90^{\circ}$.

The pole figures obtained from the simulation are shown in Fig. 11. The abovementioned evolutions of texture are predicted qualitatively well in all the results. In contrast, from a quantitative viewpoint, the simulation results are not always in agreement with the experimental results. For instance, the pole figures at the beginning of the second loading (Fig. 11 (a)) are quantitatively different: the peaks in the RD in the (0001) pole figure are much weaker in the simulation result than in the experimental result. This discrepancy eventually yields the quantitatively inaccurate simulation results at the end of the second loading especially for the angles $\theta=45,60$, and $90^{\circ}$. As described in section 3.3, the twinning rotation is determined by the reference-shear strain $\gamma_{\text {ref }}$ and a predetermined threshold value $f_{\text {th }}^{(\alpha)}$ in the present crystal plasticity model. Therefore, the simulation result of pole figures may be improved if these parameters are adjusted more carefully. However, because the physical meaning of the threshold value is scarce, it would be difficult to justify adjusted parameters. This is one of the disadvantages of the present crystal plasticity model.

Fig. 13 depicts the area and volume fractions of twins obtained from the experiment and the simulation. The experimental result of the area fraction at the beginning of the second loading is approximately $45 \%$. The area fraction at the end of the second loading is approximately $7 \%$ for the angle $\theta=0^{\circ}$, and it increases with increasing angle. Because the difference in the area fraction between the beginning and end of the second loading corresponds to detwinning activated during the second loading, this result is consistent with the EBSD measurement. The simulation results of the volume fraction are in fairly good agreement with the experimental results.

From these results, we conclude that the mesoscopic deformation behavior under the two-step loadings is also predicted qualitatively well in the present simulation. Note that only detwinning was taken into account as an additional model to simulate the deformation upon strain-path change. One of the reasons for the successful simulation would be that the activities of twinning and detwinning and the difference in the critical resolved shear stresses among the slip and twinning systems play a dominant role in the deformation behavior. Another reason may be that the second loading was tension; thus, during the second loading double twinning would be negligible and the twinning activity is small as will be discussed in section 4.6. In other words, the present crystal-plasticity model may not be able to capture the deformation characteristics in a case where the second loading is compression because double twinning and an activity of twin modes other than the pre-dominant twin mode during the first loading would be involved. Moreover, a more complex latent hardening model may also be required in 
such a case as Qiao et al. (2015) pointed out.

In the meanwhile, the effect of latent hardening on the strain hardening behavior would be more pronounced under two-step loading with $\theta \neq 0^{\circ}$ than under uniaxial loading; thus, it is presumed that the effect of the interaction matrix on the simulation accuracy can be examined in detail using the present experimental results. A further development of the model for general non-proportional loadings will be tackled in our future work.

In the following sections, the deformation mechanism is investigated in detail using the simulation results.

\subsection{Deformation mechanism in the virgin sheet}

First, the effect of the angle on the evolution of slip and twinning activities in the virgin sheet is examined. Fig. 14 presents the evolution of relative activity during monotonic tension with different angles in the virgin sheet. The relative activities are evaluated separately for the matrix and twinned regions. However, because the activities in the twinned region are negligible in the virgin sheet, only the results in the matrix region are presented here. Note that the instantaneous oscillations observed in the relative activities are because of the lattice rotation due to twinning. The activities of basal slip and prismatic slip are pronounced during tension, irrespective of the angle. As the angle increases, the activity of prismatic slip decreases slightly, whereas the twinning activity increases slightly. The pyramidal slip is also active, and its activity is almost irrespective of the angle. These results suggest that the flow stress decreases as the angle increases [Fig. 8(a)] because twinning becomes more activated instead of prismatic slip. The increase of twinning activity with increasing angle would be because the crystallographic orientations are more dispersed in the TD than in the RD [Fig. 6(a)].

\subsection{Deformation mechanism in 6\% prestrained sheet}

Fig. 15 presents the relative activities during the second loading with different angles. Because the activities in the twinned region are negligible except for detwinning, irrespective of the angle, the results in the matrix region and detwinning are displayed here. The detwinning activity at the beginning of deformation is negligible for $\theta=0^{\circ}$, whereas it increases as the angle increases. As a result, the increasing tendency of the prismatic slip activity at the beginning becomes moderate. This large activity of detwinning may be due to the following mechanism. Because of the strong basal texture and the polar characteristics of twinning, twinning is mainly activated by compressive 
stresses in the TD during the first loading (tension along the RD). Therefore, in the second loading, it becomes easier to activate detwinning as the tensile direction approaches the TD, i.e., $\theta=90^{\circ}$. It is presumed that this large activity of detwinning at the beginning eventually yields the gentle change in the work-hardening rate at the beginning for $\theta=30,45,60$, and $90^{\circ}$ [Fig. 8(b)].

Note that the activities of other slip and twinning systems are similar to those of the virgin sheet, including the increase of twinning activity with increasing angle. As a result, the volume fraction of twins at the end of the second loading increases with increasing angle as shown in Fig. 16. In contrast, it is presumed that new slip systems that are not active during the first loading would be activated in the second loading and that this tendency would become more pronounced as the angle increases. This presumption is supported by the observation that the difference in flow stress as a function of the angle is more pronounced in the $6 \%$ prestrained sheet than in the virgin sheet, despite the similar activities of the slip and twinning systems.

\subsection{Deformation mechanism in $-6 \%$ prestrained sheet}

Fig. 17 shows the relative activities during the first loading, i.e., compression. The activities of twinning and basal slip are pronounced in the matrix region in the initial stage. The prismatic slip is also activated to a small extent. Thereafter, the twinning and basal slip activities gradually decrease in the matrix region, whereas in the twinned region the basal slip activity increases.

Fig. 18 presents the relative activities during the second loading. The relative activities are significantly different from those of the virgin and 6\% prestrained sheets. In the case of $\theta=0^{\circ}$, which corresponds to typical reverse loading, the detwinning activity is the largest among the active deformation mechanisms in the initial stage. The detwinning activity starts to decrease rapidly at a strain of approximately 0.025 and then approaches zero. In contrast, the activity of prismatic slip starts increasing sharply at a strain of approximately 0.06 . This rapid transition from detwinning-dominated deformation to slip-dominated deformation yields the sigmoidal shape in the stressstrain curve [Hama and Takuda, 2012].

As we expected, the detwinning activity in the initial stage gradually decreases as the angle increases. Alternatively, the prismatic slip activity in the initial stage increases gradually. Particularly, prismatic slip is very active from the beginning of the second loading for the angles $\theta=45,60$, and $90^{\circ}$; thus, a clear transition from detwinning-dominated deformation to slip-dominated deformation as observed in the cases of the angles $\theta=0$ and $30^{\circ}$ does not occur. Moreover, the prismatic slip activity in 
the twinned region also increases with increasing angle. From the abovementioned difference in relative activities as a function of the angle, the mechanism that yields the anisotropy in the stress-strain curve is explained as follows. The yield stress in the second loading increases with increasing angle because the prismatic slip activity rather than the detwinning activity increases with increasing angle. For the same reason, the monotonic increase occurs in the stress-strain curves for the angles $\theta=45,60$, and $90^{\circ}$ because a clear transition from detwinning-dominated deformation to slip-dominated deformation does not occur. These results suggest that the shape of the stress-strain curve is primarily governed by the prismatic slip activity.

Next, the mechanism resulting in a smaller Lankford value in the $-6 \%$ prestrained sheet than in the virgin sheet is discussed. It is presumed that the twinning activity would affect the thickness strain in rolled Mg alloy sheets with strong basal texture. Steglich et al. (2014) compared the Lankford value in a rolled Mg alloy sheet between tension and compression and found that the Lankford value under compression was much smaller than that under tension. They presumed that this result occurred because of pronounced twinning activity during compression. It is hypothesized that the above presumption is applicable also to the present case: the Lankford value is smaller in the $6 \%$ prestrained sheet because the thickness decreases largely owing to the detwinning activity. To support this hypothesis, the two-step simulation is performed without allowing detwinning for the $-6 \%$ prestrained sheet. The simulation results of the Lankford value are shown in Fig. 19 (condition A). As we expected, the Lankford value is much larger than the original result, irrespective of the angle. Apparently, this result demonstrates that in rolled $\mathrm{Mg}$ alloy sheets, the thickness strain is affected by detwinning as well as twinning. The abovementioned results further suggest that it may be possible to control the thickness strain in rolled $\mathrm{Mg}$ alloy sheets somewhat by utilizing twinning and detwinning activities. This presumption is consistent with the work done by Park et al. (2013b): the stretch formability of rolled Mg alloy at room temperature was improved by imparting precompression, which might be attained because of detwinning and basal slip activities in the twinned region.

\subsection{Role of slip and twinning activities in twinned region}

The relative activities during the second loading in the $-6 \%$ prestrained sheet [Fig. 18] suggest that the effect of slip and twinning activities in the twinned region on the deformation behavior increases with increasing angle because of the decrease in the detwinning activity. To investigate this effect on the deformation behavior in detail, the two-step simulation is conducted for the $-6 \%$ prestrained sheet under the assumption 
that slip and twinning activities except for detwinning are not allowed in the twinned region. The obtained stress-strain curves are presented in Fig. 20. For reference, the experimental results are also displayed. The stress-strain curve for $\theta=0^{\circ}$ is in fairly good agreement with the experimental results, although the flow stress in the initial stage is somewhat larger than that of the experiment. This result would be because the activities in the twinned region are small. On the contrary, for $\theta=30,45,60$, and $90^{\circ}$, the flow stresses are apparently larger than those of the experimental results. This tendency is more pronounced as the angle increases. These large flow stresses occur because the twinned regions act as inclusions during deformation. These results indicate that slip and twinning activities in the twinned region play an important role in the flow stress during the second loading when detwinning is difficult to activate as in the case of $\theta=90^{\circ}$.

In contrast, the effect of slip and twinning activities in the twinned region on the Lankford value is small, as shown in Fig. 19 (condition B). Comparing the results between the case where detwinning is not allowed (condition A) and the case where slip and twinning in the twinned region are not allowed (condition $\mathrm{B}$ ), the change in the Lankford value from the original results is apparently larger in condition A than in condition B. This result indicates that the change in Lankford value associated with precompression is primarily owing to the detwinning activity and that slip and that the twinning activities in the twinned region have only a collateral effect.

\section{Conclusions}

The deformation characteristics of a rolled magnesium alloy sheet during two-step loading were studied both experimentally and numerically. The sheet was subjected to in-plane tension or compression in the rolling direction, followed by in-plane tension in different directions. The stress-strain curve, Lankford value, and texture evolution, including twinning and detwinning activities, were investigated in detail. A crystal-plasticity finite-element method was utilized to support the discussion. The results obtained in this study are summarized as follows:

(1) The prestretched sheet exhibited a monotonic increase in the stress-strain curve during the second loading irrespective of the angle between the rolling direction and the loading direction in the second loading, $\theta$, as seen in the virgin sheet. In contrast, from a quantitative viewpoint, the anisotropy in flow stress was more pronounced in the prestretched sheet than in the virgin sheet. Moreover, the change in stress in the vicinity of yielding was gentle when the angle was not zero.

(2) The precompressed sheet presented strong anisotropy in the stress-strain curve: a 
sigmoidal curve occurred for the angles $\theta=0$ and $30^{\circ}$, whereas a monotonic increase was exhibited for the angles $\theta=45,60$, and $90^{\circ}$. The yield stress increased with increasing angle, whereas the flow stress in the latter stage of deformation was rather complicated: the flow stress was the largest for the angle $\theta=90^{\circ}$ and the flow stresses for the angles $\theta=0$ and $30^{\circ}$ were larger than those of the angles $\theta=45$ and $60^{\circ}$. EBSD measurements revealed that a $\left\{\begin{array}{lllll}1 & 0 & \overline{1} & 2\end{array}\right\}$ detwinning activity decreased as the angle increased.

(3) The Lankford values for the 6\% prestrained sheet were close to those of the virgin sheet. In contrast, the Lankford values were much smaller in the $-6 \%$ prestrained sheet than in the virgin sheet, irrespective of the angle. These results indicate that the Lankford value was insensitive to pre-tension, whereas it was sensitive to precompression.

(4) The simulation results of the crystal-plasticity analysis of the two-step loading were qualitatively in good agreement with the experimental results in terms of the stressstrain curve, Lankford value, texture evolution, and twin fraction. However, the simulation results were rather poor when the compressive strain imparted in the first loading was small. Moreover, the simulation results of the Lankford values were not in quantitative agreement with the experimental results. These results suggest that the present simulation model of two-step loading still has some room for further improvement in terms of quantitatively accurate predictions.

(5) The simulation results showed that the pronounced anisotropy in the stress-strain curve observed during the second loading could be illustrated from the perspective of the detwinning activity. Particularly, in the precompressed sheet, the decrease in the detwinning activity with increasing angle yielded both the increase in yield stress and the monotonic increase in the stress-strain curve. Moreover, the simulation results also suggest that in the precompressed sheet, the shape of the stress-strain curve was primarily governed by prismatic slip.

(6) It is presumed from the numerical experiments that slip and twinning activities in the twinned region played an important role in the stress-strain response during the second loading. In contrast, it is also suggested that their effect on the Lankford value was rather small. Alternatively, the significant decrease in the Lankford value in the $6 \%$ prestrained sheet would be primarily owing to the detwinning activity.

\section{Acknowledgements}


The authors appreciate the help of Dr. Tsutomu Tanaka and Mr. Sohei Uchida of TRI Osaka with the EBSD measurement. The help of Mr. Shinichi Hatakeyama of Kyoto University with the measurements of twin fractions and Lankford values is acknowledged. T.H. is grateful for the financial support from the Photon and Quantum Basic Research Coordinated Development Program from the Ministry of Education, Culture, Sports, Science and Technology, Japan, and JSPS KAKENHI Grant Number 26289271.

\section{References}

Abdolvand,H., Majkut,M., Oddershede,J., Schmidt,S., Lienert,U., Diak,B.J., Withers,P.J., and Daymond,M.R., 2015. On the deformation twinning of Mg AZ31B: A threedimensional synchrotron X-ray diffraction experiment and crystal plasticity finite element model. International Journal of Plasticity, 70, 77-97.

Askari,H., Young,J., Field,D., Kridli,G., Li,D., and Zbib,H., 2014. A study of the hot and cold deformation of twin-rolled cast magnesium alloy AZ31. Philosophical magazine, 94-4, 381-403.

Balík,J., Dobroň,P., Chmelík,F., Kužel,R., Drozdenko,D., Bohlen,J., Letzig,D., and Lukáč,P., 2016. Modeling of the work hardening in magnesium alloy sheets. International Journal of Plasticity, 76, 166-185.

Barlat,F., Ha,J.J., Gracio,J.J., Lee,M.-G., Rauch,E.F., and Vincze,G., 2013. Extension of homogeneous anisotropic hardening model to cross-loading with latent effects. International journal of plasticity, 46, 130-142.

Bertin,N., Tomé,C.N., Beyerlein,I.J., Barnett,M.R., and Capolungo, L., 2014. On the strength of dislocation interactions and their effect on latent hardening in pure Magnesium. International Journal of Plasticity 62, 72-92.

Cheng,J. and Ghosh,S., 2015. A crystal plasticity FE model for deformation with twin nucleation in magnesium alloys, International Journal of Plasticity. 67, 148-170.

Dogan,E., Vaughan,M.W., Wang,S.J., Karamana,I., and Proust,G., 2015. Role of starting texture and deformation modes on low-temperature shear formability and shear localization of Mg-3Al-1Zn alloy. Acta Materialia, 89, 408-422.

Easton,M., Beer,A., Barnett,M., Davies,C., Dunlop,G., Durandet,Y., Blacket,S., Hilditch,T., and Beggs,P., 2008. Magnesium alloy applications in automotive structures. JOM - The Journal of the Minerals, Metals \& Materials Society, 60-11, 57-62.

Fernández,A., Jérusalem,A., Gutiérrez-Urrutia,I., and Pérez-Prado,M.T., 2013. Three-dimensional investigation of grain boundary-twin interactions in a Mg AZ31 alloy by electron backscatter diffraction and continuum modeling. Acta Materialia, 61, 


\section{9-7692.}

Frankel,G.S., 2015. Magnesium alloys Ready for the road. Nature Materials, 14, 1189-1190.

Gérard,C., Cailletaud,G., and Bacroix,B., 2013. Modeling of latent hardening produced by complex loading paths in FCC alloys. International journal of plasticity, 42, 194-212.

Ghaffari Tari, D., Worswick, M.J., Ali, U., and Gharghouri, M.A., 2014. Mechanical response of AZ31B magnesium alloy: Experimental characterization and material modeling considering proportional loading at room temperature. International journal of plasticity, 55, 247-267.

Graff,S., Brocks,W., and Steglich,D., 2007. Yielding of magnesium: from single crystal to polycrystalline aggregates. International Journal of Plasticity 23, 1957-1978.

Gu,C.F., Toth,L.S., and Hoffman,M., 2014. Twinning effects in a polycrystalline magnesium alloy under cyclic deformation. Acta Materialia, 62, 212-224.

Guo,T., Siska,F., and Barnett,M.R., 2016. Distinguishing between slip and twinning events during nanoindentation of magnesium alloy AZ31. Scripta Materialia, 110, 10-13.

Hahm,J.H., and Kim,K.H., 2008. Anisotropic work hardening of steel sheets under plane stress. International journal of plasticity, 24, 1097-1127.

Hama,T., and Takuda,H., 2011. Crystal-Plasticity Finite-Element Analysis of Inelastic Behavior During Unloading in a Magnesium Alloy Sheet. International Journal of Plasticity, 27, 1072-1092.

Hama,T., and Takuda,H., 2012a. Crystal Plasticity Finite-Element Simulation of Work-Hardening Behavior in a Magnesium Alloy Sheet under Biaxial Tension. Computational Materials Science, 51, 156-164.

Hama,T., and Takuda,H., 2012b. Crystal plasticity finite-element simulation of deformation behavior in a magnesium alloy sheet considering detwinning. Steel Research International Special Edition, 1115-1118.

Hama,T., Kariyazaki,Y., Hosokawa,N., Fujimoto,H., and Takuda,H., 2012. Work-hardening behaviors of magnesium alloy sheet during in-plane cyclic loading. Materials Science and Engineering A, 551, 209-217.

Hama,T., Kitamura,N., and Takuda,H., 2013. Effect of twinning and detwinning on inelastic behavior during unloading in a magnesium alloy sheet. Materials Science and Engineering A, 583, 232-241.

Hama,T., Nagao,H., Kuchinomachi,Y., and Takuda,H., 2014. Effect of pre-strain on work-hardening behavior of magnesium alloy sheets upon cyclic loading. Materials 
Science and Engineering A, 591, 69-77.

Hama,T., Matsudai,R., Kuchinomachi,Y., Fujimoto,H., and Takuda,H., $2015 a$. Non-linear deformation behavior during unloading in various metal sheets. ISIJ International, 55, 1067-1075.

Hama,T., Mayama,T., and Takuda,H., 2015b. Deformation behavior of a Magnesium alloy sheet with random crystallographic orientations under various loading paths. Romanian Journal of Technical Sciences -Applied Mechanics, 60, 16 pages.

Hiura,F., and Niewczas,M., 2015. Latent hardening effect under self- and coplanar dislocation interactions in Mg single crystals. Scripta Materialia, 106, 17-20.

Hoc,T., and Forest,S., 2001. Polycrystal modelling of IF-Ti steel under complex loading path. International journal of plasticity, 17, 65-85.

Holmedal,B., Van Houtte,P., and An,Y., 2008. A crystal plasticity model for strain-path changes in metals. International journal of plasticity, 24, 1360-1379.

Jiang,L., Jonas,J.J., Mishra,R.K., Luo,A.A., Sachdev,A.K., and Godet,S., 2007. Twinning and texture development in two Mg alloys subjected to loading along three different strain paths. Acta Materialia, 55, 3899-3910.

Kabirian,F., Khan,A.S., and Gnaupel-Herlod,T., 2015. Visco-plastic modeling of mechanical responses and texture evolution in extruded AZ31 magnesium alloy for various loading conditions. International Journal of Plasticity, 68, 1-20.

Kim,K.H., and Yin,J.J., 1997. Evolution of anisotropy under plane stress. Journal of the mehcanics and physics of solids, 45-5, 841-851.

Kitayama,K., Tomé,C.N., Rauch,E.F., Gracio,J.J., and Barlat,F., 2013. A crystallographic dislocation model for describing hardening of polycrystals during strain path changes. Application to low carbon steels. International journal of plasticity, 46 , 54-69.

Knezevic,M., Levinson,A., Harris,R., Mishra,R.K., Doherty,R.D., and Kalidindi,S.R., 2010. Deformation twinning in AZ31: Influence on strain hardening and texture evolution. Acta Materialia, 58, 6230-6242.

Kulekci,M.K., 2008. Magnesium and its alloys applications in automotive industry.International Journal of Advanced Manufacturing Technology, 39, 851-865.

Kurukuri,S., Worswick,M.J., Tari,D.G., and Mishra,R.K., 2014. Rate sensitivity and tension-compression asymmetry in AZ31B magnesium alloy sheet. Philosophical transactions of the royal society A, 372, 20130216.

Kuwabara,T., 2007. Advances in experiments on metal sheets and tubes in support of constitutive modeling and forming simulations. International Journal of Plasticity 23, 385-419. 
Lee,S.Y., and Gharghouri,M.A., 2013. Pseudoelastic behavior of magnesium alloy during twinning-dominated cyclic deformation. Materials Science and Engineering A, 572, 98-102.

Li,D.S., Ahzi,S., M’Guil,S., Wen,W., Lavender,C., and Khaleel,M.A., 2014. Modeling of deformation behavior and texture evolution in magnesium alloy using the intermediate $\phi$-model. International Journal of Plasticity, 52, 77-94.

Liu,Y., and Wei,Y., 2014. A polycrystal based numerical investigation on the temperature dependence of slip resistance and texture evolution in magnesium alloy AZ31B. International Journal of Plasticity, 55, 80-93.

Lou,X.Y., Li,M., Boger,R.K., Agnew,S.R., and Wagoner,R.H., 2007. Hardening evolution of AZ31B Mg sheet. International Journal of Plasticity 23, 44-86.

Mánik,T., Holmedal,B., and Hopperstad,O.S., 2015. Strain-path change induced transients in flow stress, work hardening and r-values in aluminum. International journal of plasticity, 69, 1-20.

Mayama,T., Ohashi,T., Tadano,Y., and Hagihara,K., 2015. Crystal plasticity analysis of development of intragranular misorientations due to kinking in HCP single crystals subjected to uniaxial compressive loading. Materials Transactions, 56-7, 963-972.

Nguyen,N.-T., Seo,O.S., Lee,C.A., Lee,M.-G., Kin,J.-H., and Kim,H.Y., 2014. Mechanical behavior of AZ31B Mg alloy sheets under monotonic and cyclic loadings at room and moderately elevated temperatures. Materials, 7, 1271-1295.

Oppedal,A.L., El Kadiri,H., Tome,C.N., Vogel,S.C., and Horstemeyer,M.F., 2013. Anisotropy in hexagonal close-packed structures: improvements to crystal plasticity approaches applied to magnesium alloy. Philosophical Magazine, 93-35, 4311-4330.

Pan,J., and Rice,J.R., 1983. Rate sensitivity of plastic flow and implications for yield-surface vertices. International Journal of Solids and Structures, 19-11, 973-987.

Park,S.H., Hong,S.-G., and Lee,C.S., 2013a. In-plane anisotropic deformation behavior of rolled Mg-3Al-1Zn alloy by initial \{10-12\} twins. Materials Science and Engineering A, 570, 149-163.

Park,S.H., Hong,S.-G., and Lee,C.S., 2013b. Enhanced stretch formability of rolled Mg-3Al-1Zn alloy at room temperature by initial $\{10-12\}$ twins. Materials Science and Engineering A, 578, 271-276.

Park,S.H., Hong,S.-G., Lee,J.H., and Huh,Y.-H., 2015. Texture evolution of rolled Mg-3Al-1Zn alloy undergoing a $\{10-12\}$ twinning dominant strain path change. Journal of Alloys and Compounds, 646, 573-579.

Peeters,B., Seefeldt,M., Teodosiu,C., Kalidindi,S.R., Van Houtte,P., and Aernoudt,E., 2001. Work-hardening/softening behavior of BCC polycrystals during changing strain 
paths: I. An integrated model based on substructure and texture evolution, and its prediction of the stress-strain behavior of an IF steel during two-stage strain paths. Acta Materialia, 49, 1607-1619.

Pierce,D., Shi,C.F., and Needleman,A., 1984. A tangent modulus method for rate dependent solids. Computers \& Structures, 18-5, 875-887.

Proust,G., Tomé,C.N., Jain,A., Agnew,S.R., 2009. Modeling the effect of twinning and detwinning during strain-path changes of magnesium alloy AZ31. International Journal of Plasticity 25, 861-880.

Qiao,H., Agnew,S.R., and Wu,P.D., 2015. Modeling twinning and detwinning behavior of $\mathrm{Mg}$ alloy ZK60A during monotonic and cyclic loading. International Journal of Plasticity, 65, 61-84.

Rauch,E.F., Gracio,J.J., Barlat,F., Vincze,G., 2011. Modelling the plastic behavior of metals under complex loading conditions. Modelling and Simulation in Materials Science and Engineering, 19 (035009), 1-18.

Sánchez-Martín,R., Pérez-Prado,M.T., Segurado,J., and Molina-Aldareguia,J.M., 2015. Effect of indentation size on the nucleation and propagation of tensile twinning in pure magnesium. Acta Materialia, 93, 114-128.

Sarker,D., and Chen,D.L., 2014. Dependence of compressive deformation on pre-strain and loading direction in an extruded magnesium alloy: Texture, twinning and de-twinning. Materials Science and Engineering A, 596,134-144.

Sarker,D., Friedman,J., and Chen,D.L., 2014. Influence of pre-strain on de-twinning activity in an extruded AM30 magnesium alloy. Materials Science and Engineering A, 605, 73-79.

Segurado,J., Lebensohn,R.A., LLorca,J., Tomé,C.N., 2012. Multiscale modeling of plasticity based on embedding the viscoplastic self-consistent formulation in implicit finite elements. International Journal of Plasticity, 28, 124-140.

Shi,Z.-Z., Zhang,Y., Wagner,F., Richeton,T., Juan,P.-A., Lecomte,J.-S., Capolungo,L., and Berbenni,S., 2015. Sequential double extension twinning in a magnesium alloy: Combined statistical and micromechanical analyses. Acta Materialia, 96, 333-343.

Steglich,D., Jeong,Y., Andar,M.O., and Kuwabara,T., 2012. Biaxial deformation behavior of AZ31 magnesium alloy: Crystal-plasticity-based prediction and experimental validation. International journal of solids and structures, 49, 3551-3561.

Steglich,D., Tian,X., Bohlen,J., and Kuwabara,T., 2014. Mechanical testing of thin sheet magnesium alloys in biaxial tension and uniaxial compression. Experimental mechanics, 54, 1247-1258.

Suh,J., Victoria-Hernandez,J., Letzig,D., Golle,R., Yi,S., Bohlen,J., and Volk,W., 
2015. Improvement in cold formability of AZ31 magnesium alloy sheets processed by equal channel angular pressing. Journal of Materials Processing Technology, 217, 286-293.

Taub,A.I., and Luo,A.A., 2015. Advanced lightweight materials and manufacturing processes for automotive applications. MRS Bulletin, 40, 1045-1053.

Teodosiu,C., Hu,Z., 1998. Microstructure in the continuum modeling of plastic anisotropy. In: 19th Riso International Symposium on Materials Science Proceedings, Roskilde, pp. 149-168.

Van Houtte,P., 1978. Simulation of the rolling and shear texture of brass by the Taylor theory adapted for mechanical twinning. Acta Metallurgica 26, 591-604.

Wang,H., Yan,Y., Wan,M., Wu,X., 2012. Experimental investigation and constitutive modeling for the hardening behavior of 57540 aluminum alloy sheet under two-stage loading. International journal of solids and structures, 49, 3693-3710.

Wang,H., Wu,P.D., and Wang,J., 2013a. Modeling inelastic behavior of magnesium alloys during cyclic loading-unloading. International Journal of Plasticity, 47, 49-64.

Wang,H., Wu,P.D., Wang,J., Tome,C.N., 2013b. A crystal plasticity model for hexagonal close packed (HCP) crystals including twinning and de-twinning mechanisms. International Journal of Plasticity, 49, 36-52.

Wen,W., Borodachenkova,M., Tomé,C.N., Vincze,G., Rauch,E.F., Barlat,F., and Grácio,J.J., 2015. Mechanical behavior of Mg subjected to strain path changes: Experiments and modeling. International Journal of Plasticity, 73, 171-183.

Wu,W., Qiao,H., An,K., Guo,X., Wu,P.D., and Liaw,P.K., 2014. Investigation of deformation dynamics in a wrought magnesium alloy. International Journal of Plasticity, 62, 105-120.

Xin,Y., Zhou,X., Lv,L., and Liu,Q., 2014. The influence of a secondary twin on the detwinning deformation of a primary twin in Mg-3Al-1Zn alloy. Materials Science and Engineering A, 606, 81-91.

Xin,Y., Zhou,X., Wu,Y., Yu,H., and Liu,Q., 2015. Deformation behavior and mechanical properties of composite twin structures under different loading paths. Materials Science and Engineering A, 640, 118-128.

Xu,S., Liu,Tianmo, Ding,X., and Zeng,W., 2014. Reducing the anisotropy of a pre-twinned hot-rolled Mg-3Al-1Zn alloy by plane-strain compression. Materials Science and Engineering A, 592, 230-235. 
List of Figure and Table captions

Table 1 Mechanical properties of the rolled Mg alloy sheet obtained under tension *

Table 2 Latent-hardening parameters, $q_{\alpha \beta}$, used in the present study [Graff et al., 2007].

Table 3 Identified parameters.

Fig.1 Schematic diagram of two-step loading test.

Fig. 2 Geometries of (a) large and (b) small samples in millimeters.

Fig. 3 Schematic diagram of experimental setup used in the first loading.

Fig. 4 Photograph of small sample preparation.

Fig. 5 Finite-element model used in simulation.

Fig.6 Initial crystallographic orientations of the virgin sheet. (a) Pole figures and (b) inverse pole figure map.

Fig. 7 Results of parameter identification. (a) Stress-strain curves obtained under tension and compression, (b) those under compression-tension, and (c) evolution of Lankford value under tension in the RD.

Fig. 8 True stress-logarithmic strain curves obtained in the second loading with different angles in the (a) virgin sheet, (b) $6 \%$ prestrained sheet, (c) $-3 \%$ prestrained sheet, and (d) $-6 \%$ prestrained sheet. Symbols and solid lines are the experimental and simulation results, respectively.

Fig. 9 Evolution of work-hardening rate during the second loading. The results of the $3 \%$ prestrained sheet with (a) $\theta=0,30$ and $45^{\circ}$ and (b) $\theta=60$ and $90^{\circ}$, and the results of the $-6 \%$ prestrained sheet with (c) $\theta=0,30$ and $45^{\circ}$ and (d) $\theta=60$ and $90^{\circ}$. Symbols and solid lines are the experimental and simulation results, respectively.

Fig. 10 Lankford values measured after the second loading was completed.

Fig. 11 Experimental and simulation results of pole figure for the $-6 \%$ prestrained sheet measured (a) before the second loading and after the second loading for $\theta$ of (b) 0 , (c) 30, (d) 45, (e) 60, and (f) $90^{\circ}$.

Fig. 12 Experimental results of inverse pole figure maps for the $-6 \%$ prestrained sheet measured (a) before the second loading and after the second loading for $\theta$ of (b) 0 , (c) 30, (d) 45, (e) 60, and (f) $90^{\circ}$.

Fig. 13 Area/volume fractions of twins measured before and after the second loading in the $-6 \%$ prestrained sheet.

Fig. 14 Evolutions of relative activity in matrix region during monotonic tension in the virgin sheet at $\theta$ of (a) 0 , (b) 30, (c) 45, (d) 60, and (e) $90^{\circ}$.

Fig. 15 Evolutions of relative activity in matrix region during the second loading in the $6 \%$ prestrained sheet at $\theta$ of (a) 0 , (b) 30, (c) 45, (d) 60, and (e) $90^{\circ}$. 
Fig. 16 Simulation results of volume fractions of twins measured before and after the second loading in the $6 \%$ prestrained sheet.

Fig. 17 Evolutions of relative activity during the first loading in the $-6 \%$ prestrained sheet.

Fig. 18 Evolutions of relative activity during the second loading in the $-6 \%$ prestrained sheet at $\theta$ of (a) 0, (b) 30, (c) 45, (d) 60, and (e) $90^{\circ}$.

Fig. 19 Simulation results of Lankford value for the $-6 \%$ prestrained sheet with different numerical conditions. Condition A denotes the simulation where detwinning is not allowed, and condition B denotes the simulation where slip and twinning activities in the twinned region are not allowed.

Fig. 20 Simulation results of stress-strain curve for the $-6 \%$ prestrained sheet where slip and twinning activities in the twinned region are not allowed. 
Table 1 Mechanical properties of the rolled Mg alloy sheet obtained under tension *

\begin{tabular}{ccccc}
\hline$E \mathrm{GPa}$ & $\sigma_{\mathrm{T}} \mathrm{MPa}$ & $\sigma_{0.2} \mathrm{MPa}$ & $F \mathrm{MPa}$ & $n$ \\
43.0 & 271 & 188 & 440 & 0.175 \\
\hline
\end{tabular}

$E$ is Young's modulus, $\sigma_{\mathrm{T}}$ is the ultimate tensile strength, and $\sigma_{0.2}$ is the $0.2 \%$ proof stress. The true stress-logarithmic strain curve was approximated using the equation $\sigma=F \varepsilon^{n}$. 
Table 2. Latent-hardening parameters, $q_{\alpha \beta}$, used in the present study [Graff et al., 2007].

\begin{tabular}{lcccc}
\hline & Basal & Prismatic & Pyramidal $<\mathrm{c}+\mathrm{a}>$ & Twinning \\
Basal & 0.2 & 0.5 & 0.5 & 0.5 \\
Prismatic & 0.2 & 0.2 & 0.2 & 0.5 \\
Pyramidal $<\mathrm{c}+\mathrm{a}>$ & 1.0 & 1.0 & 0.2 & 0.25 \\
Twinning & 1.0 & 1.0 & 0.2 & 0.25 \\
\hline
\end{tabular}


Table 3 Identified parameters.

\begin{tabular}{ccccccc}
\hline & & Basal & Prismatic & Pyramidal $<\mathrm{c}+\mathrm{a}>$ & Twinning & Detwinning \\
\hline$\tau_{0}$ & $/ \mathrm{MPa}$ & 25 & 95 & 100 & 40 & 35 \\
\hline$\tau_{\infty}$ & $/ \mathrm{MPa}$ & - & 230 & 190 & - & - \\
\hline$h_{0}$ & $/ \mathrm{MPa}$ & 10 & 1200 & 1200 & 5 & 5 \\
\hline
\end{tabular}




\section{First loading}

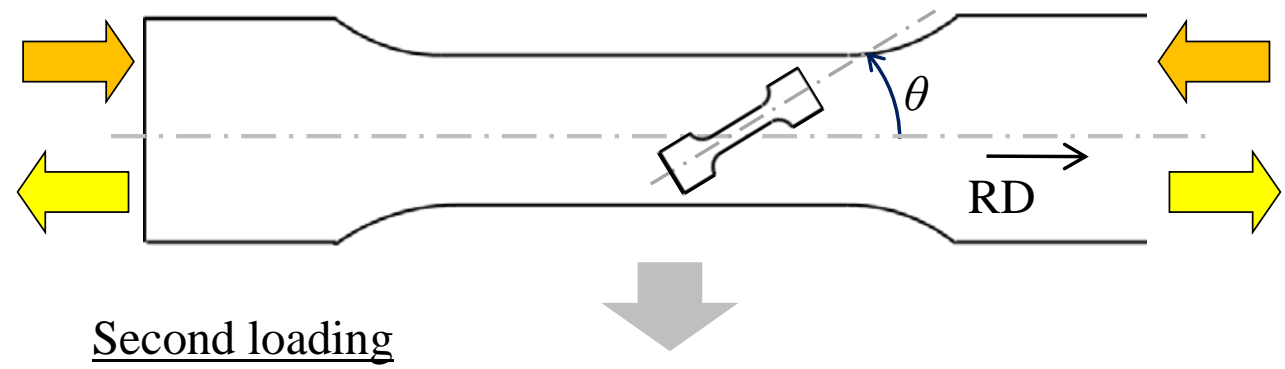

$$
\begin{aligned}
& \longleftrightarrow \square \square \\
& \theta=0,30,45,60 \text {, and } 90^{\circ}
\end{aligned}
$$

Fig.1 Schematic diagram of two-step loading test. 


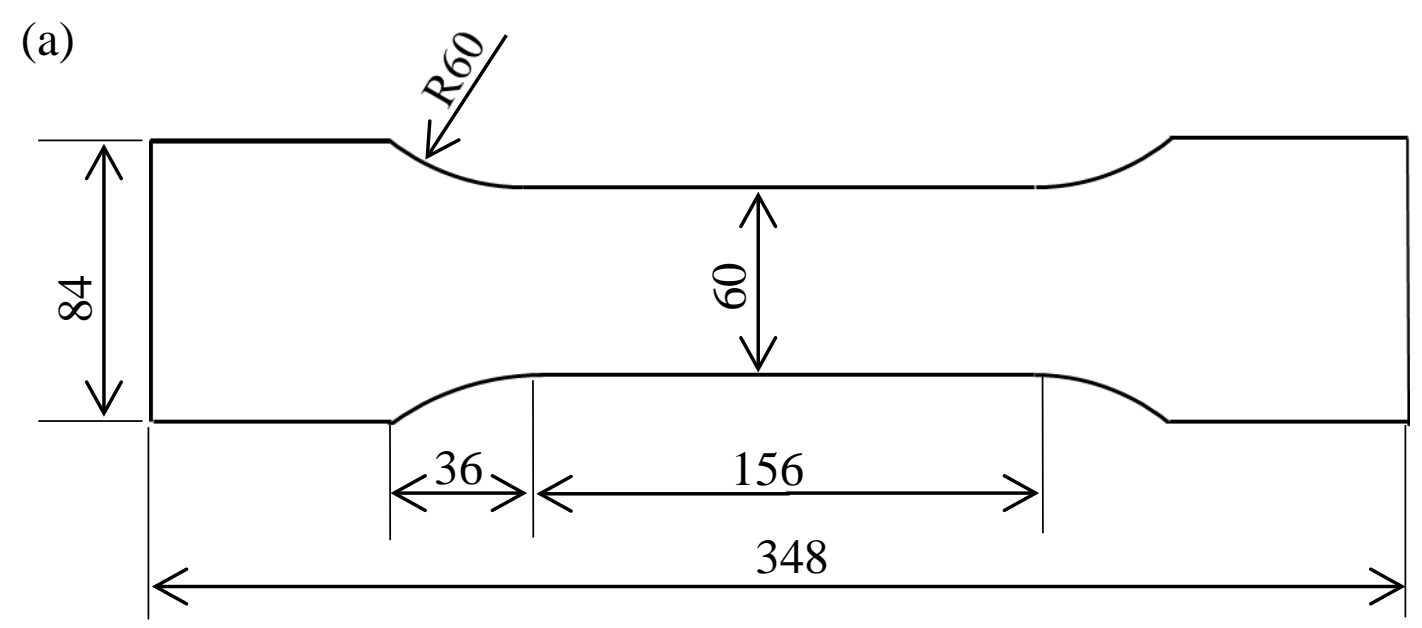

(b)

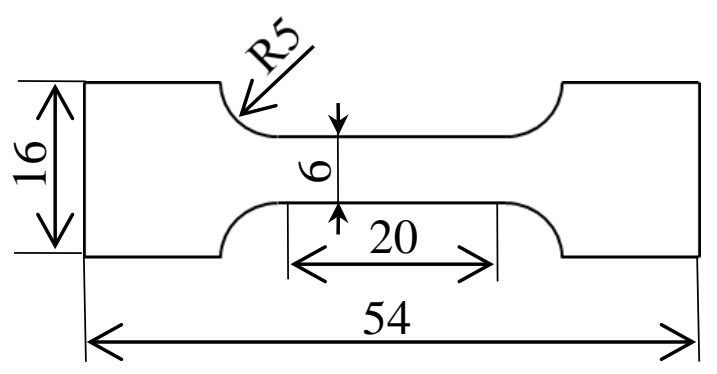

Fig. 2 Geometries of (a) large and (b) small samples in millimeters. 


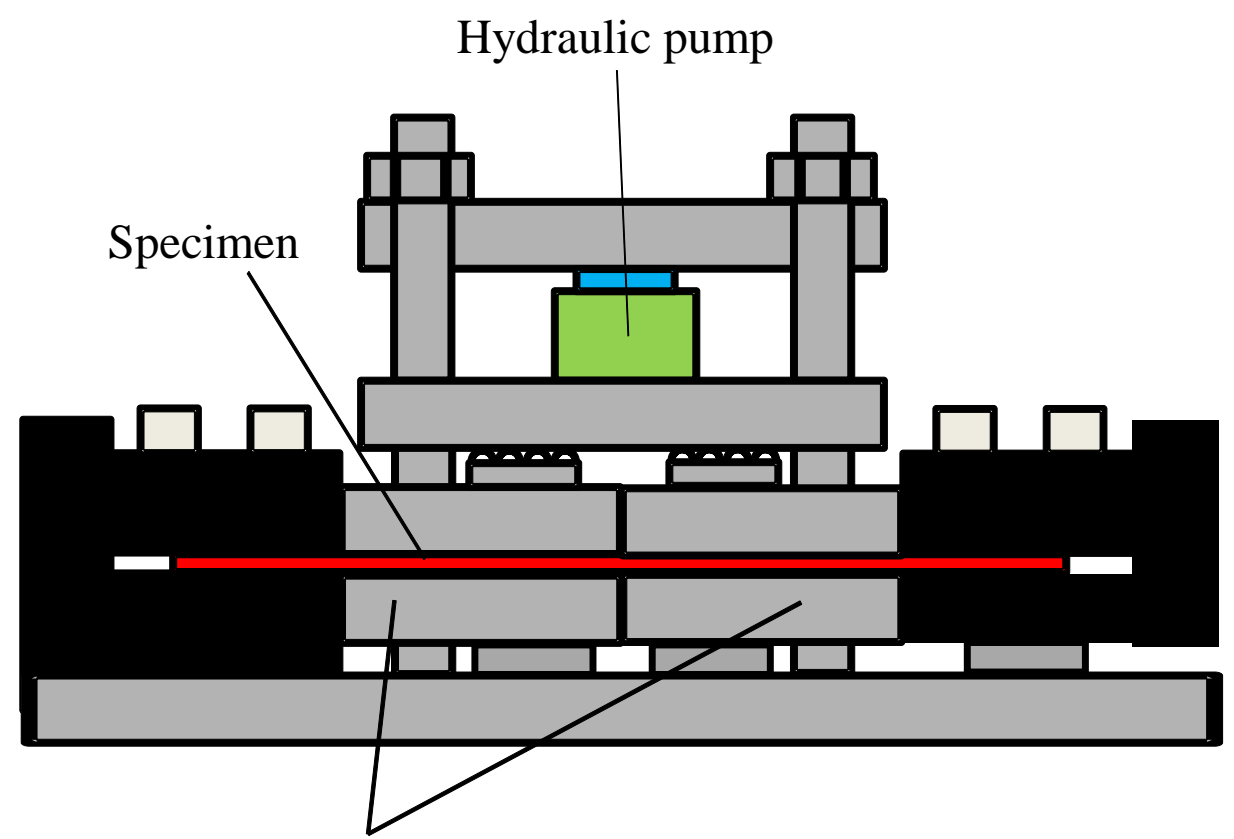

Comb-shaped dies

Fig. 3 Schematic diagram of experimental setup used in the first loading. 


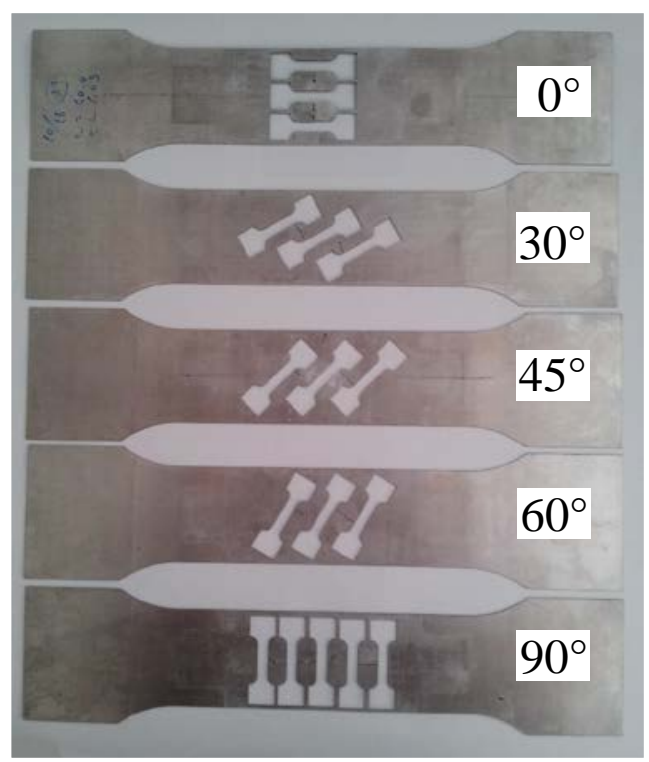

Fig. 4 Photograph of small sample preparation. 


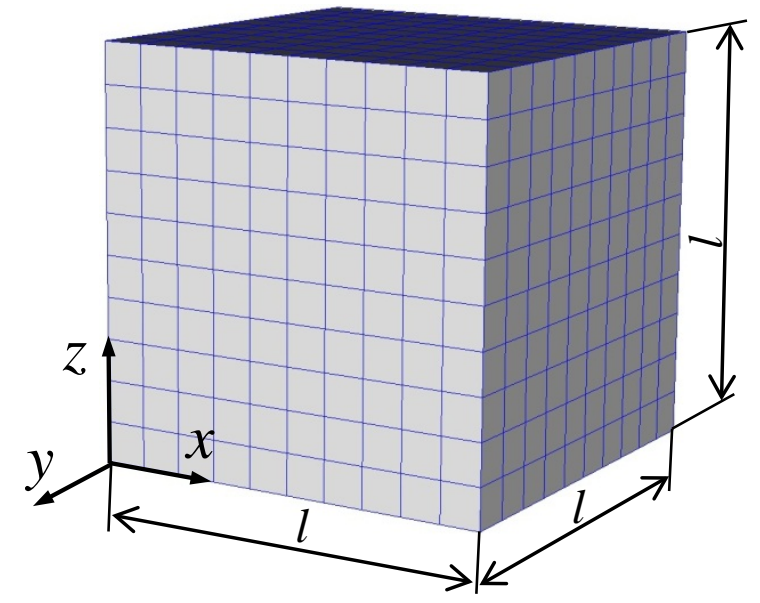

Fig. 5 Finite-element model used in simulation. 
(a)
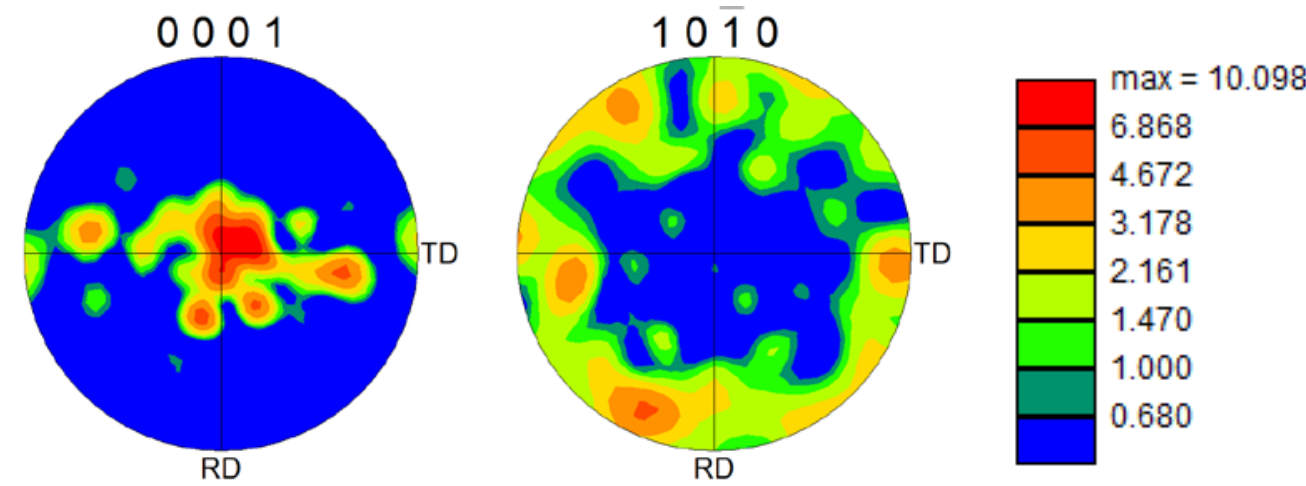

(b)
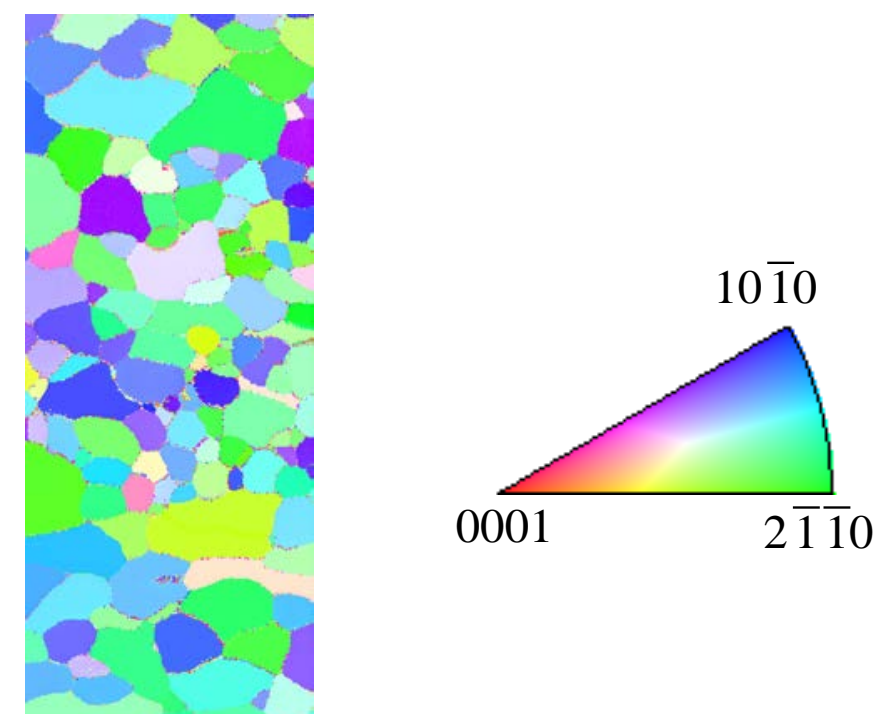

$\mathrm{ND} \stackrel{\mathrm{20 \mu \textrm {m }}}{\longrightarrow}$

TD

Fig.6 Initial crystallographic orientations of the virgin sheet. (a) Pole figures and (b) inverse pole figure map. 
(a)

- Experiment

- - Original Simulation

-.- Two-step Simulation

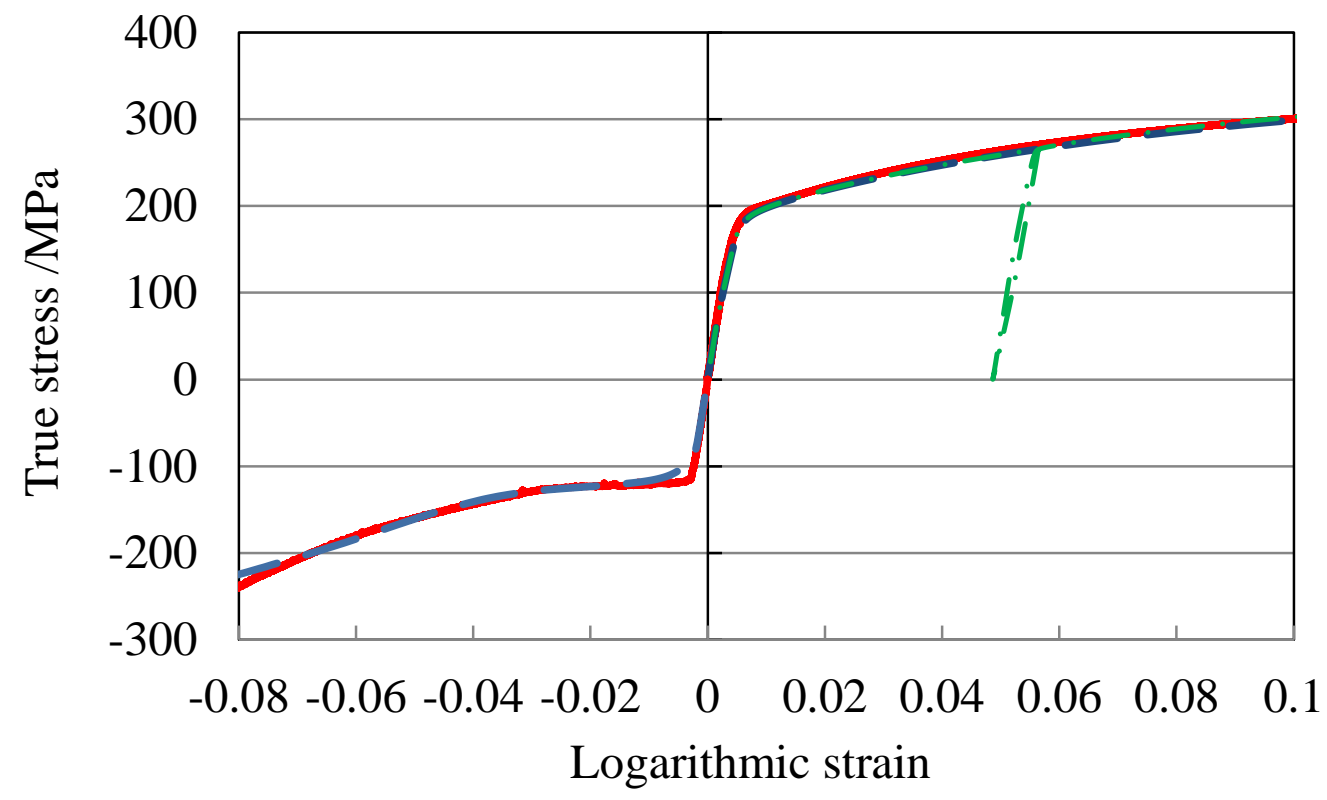

(b)

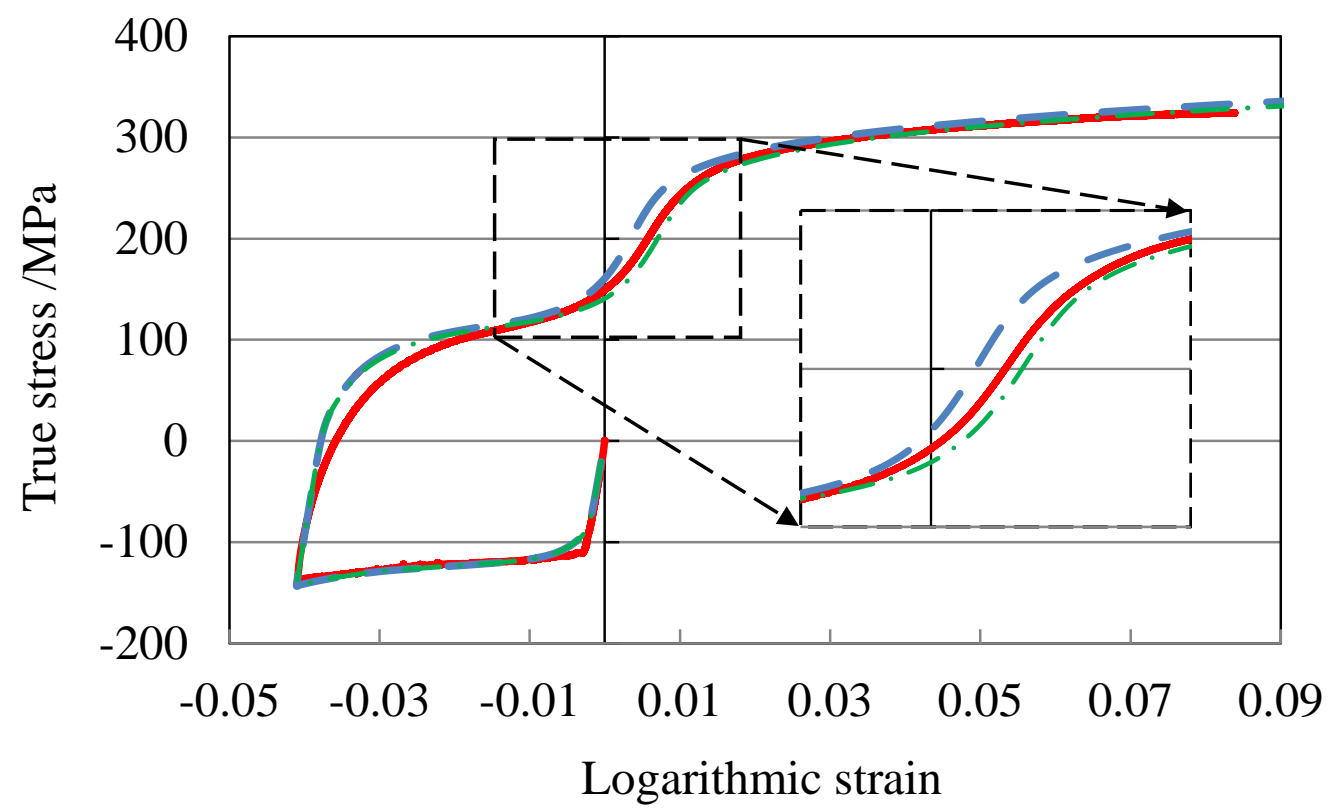

Fig. 7 Results of parameter identification. (a) Stress-strain curves obtained under tension and compression, (b) those under compression-tension, and (c) evolution of Lankford value under tension in the RD. 
(c)

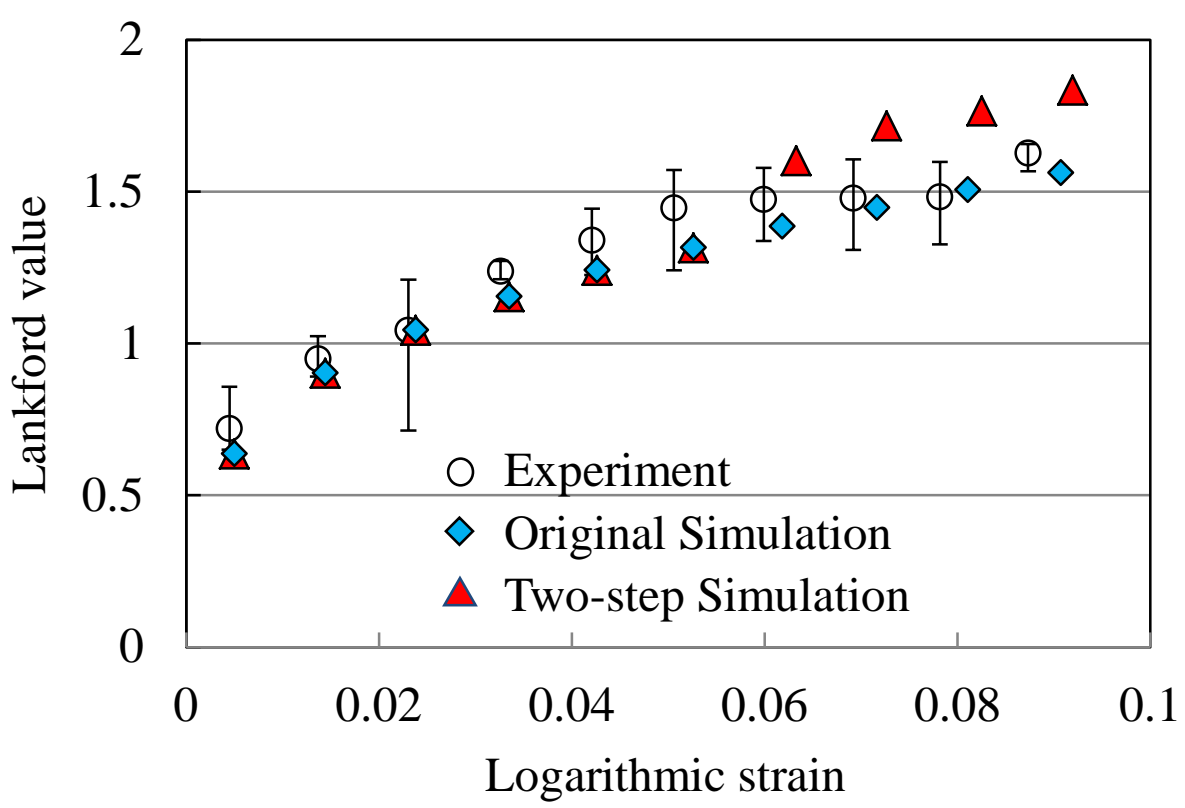

Fig. 7 Results of parameter identification. (a) Stress-strain curves obtained under tension and compression, (b) those under compression-tension, and (c) evolution of Lankford value under tension in the RD. 
(a)

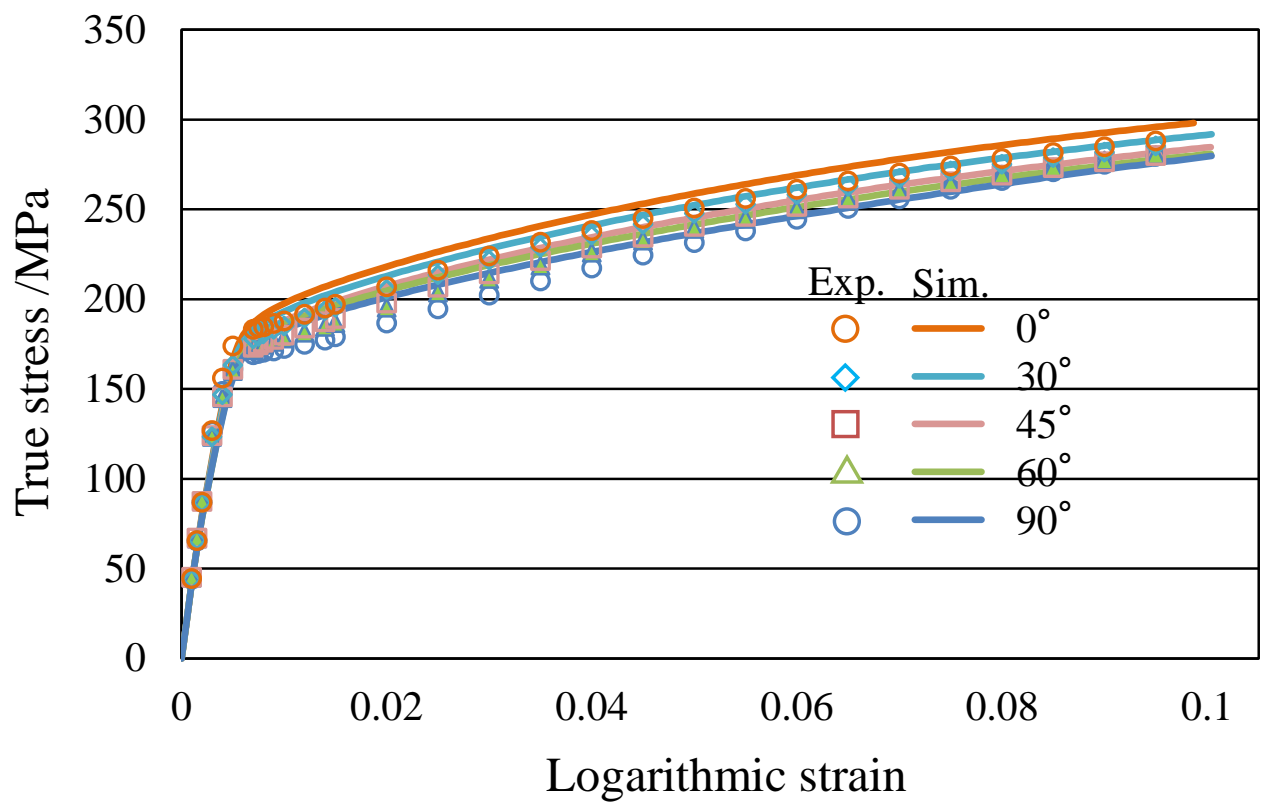

(b)

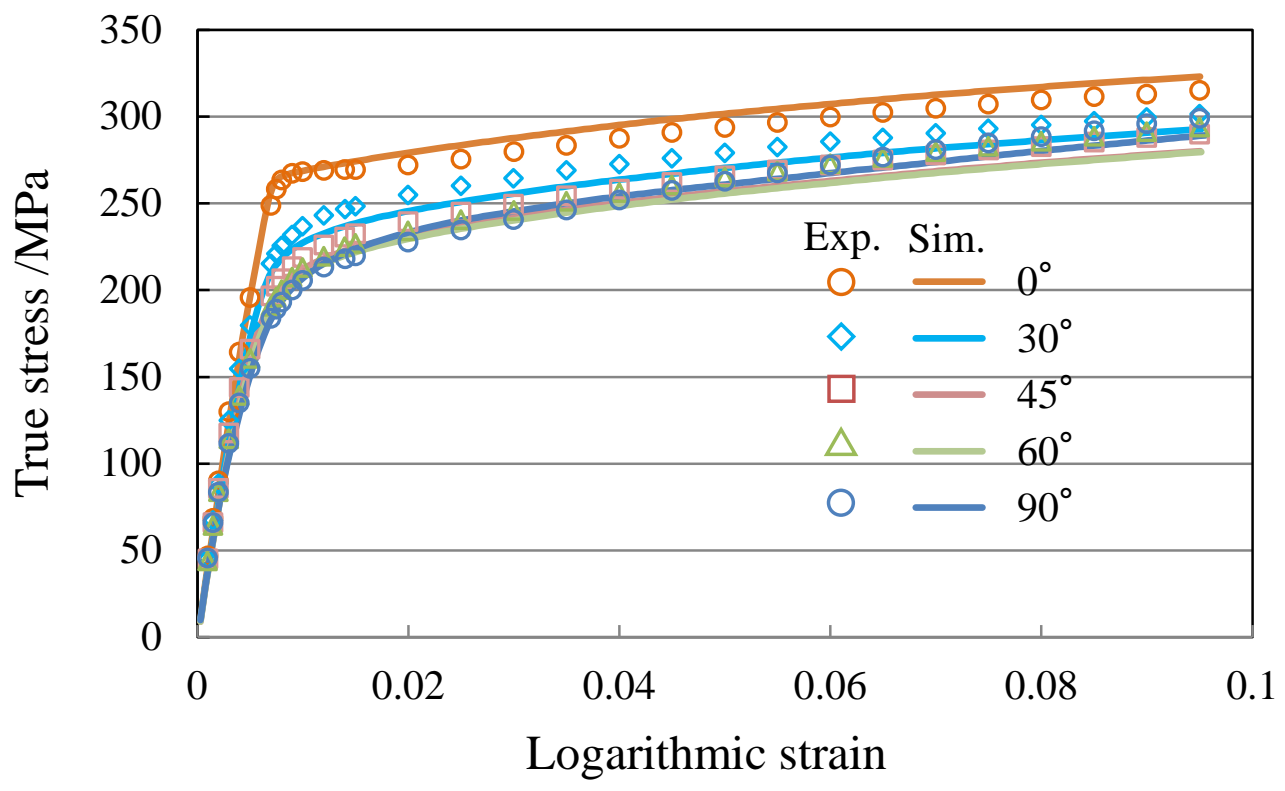

Fig. 8 True stress-logarithmic strain curves obtained in the second loading with different angles in the (a) virgin sheet, (b) 6\% prestrained sheet, (c) -3\% prestrained sheet, and (d) $-6 \%$ prestrained sheet. Symbols and solid lines are the experimental and simulation results, respectively. 
(c)

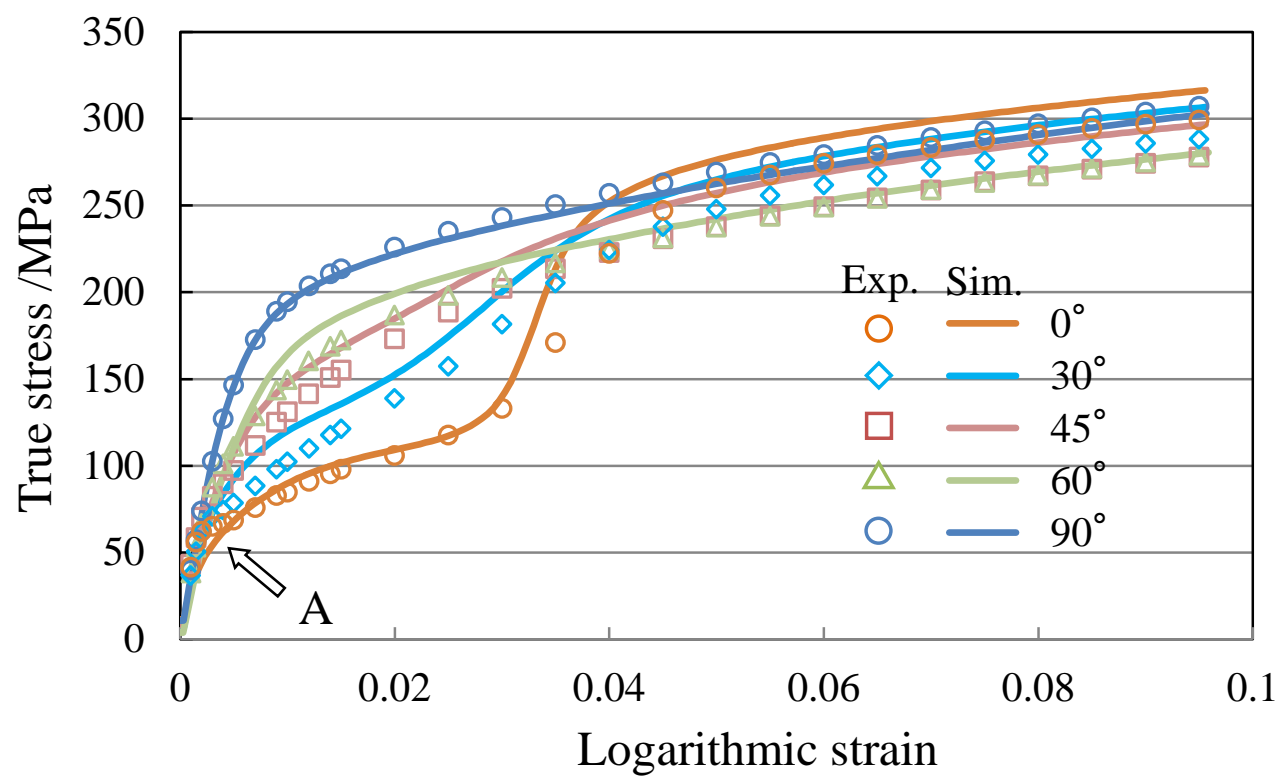

(d)

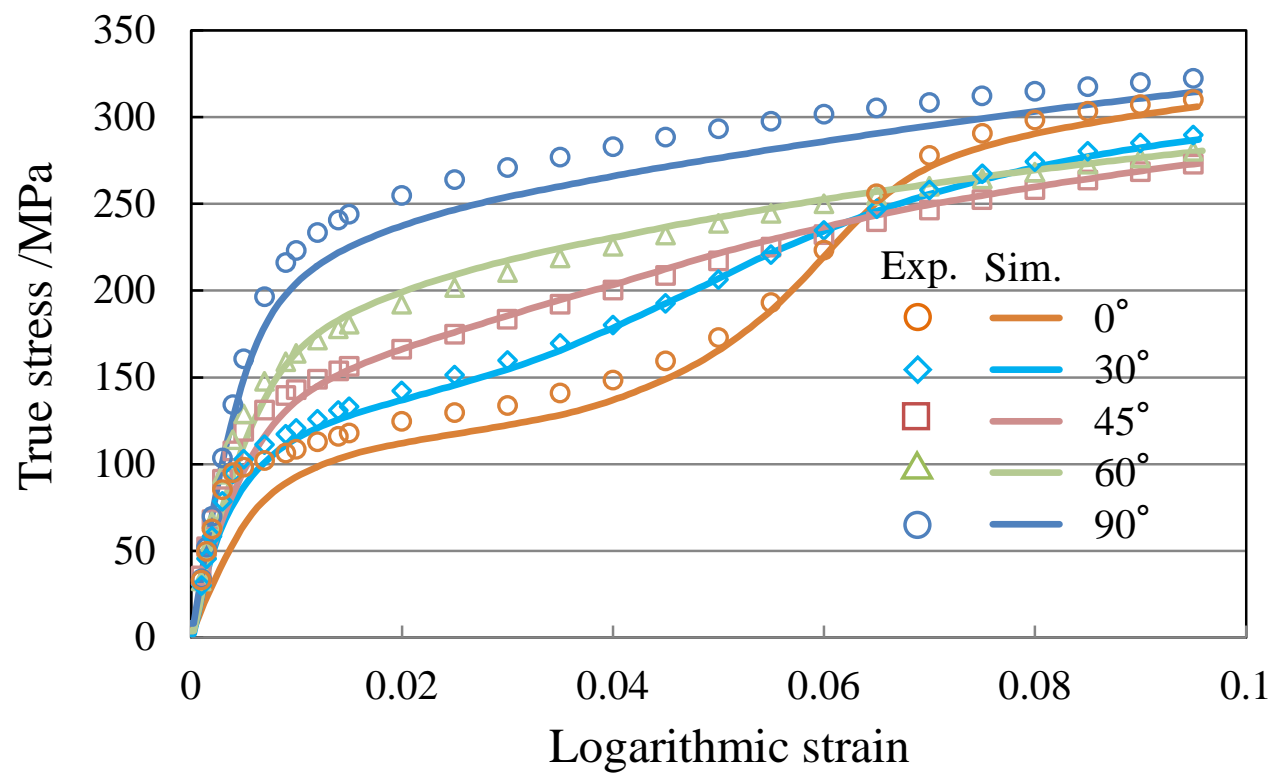

Fig. 8 True stress-logarithmic strain curves obtained in the second loading with different angles in the (a) virgin sheet, (b) 6\% prestrained sheet, (c) -3\% prestrained sheet, and (d) $-6 \%$ prestrained sheet. Symbols and solid lines are the experimental and simulation results, respectively. 
(a)

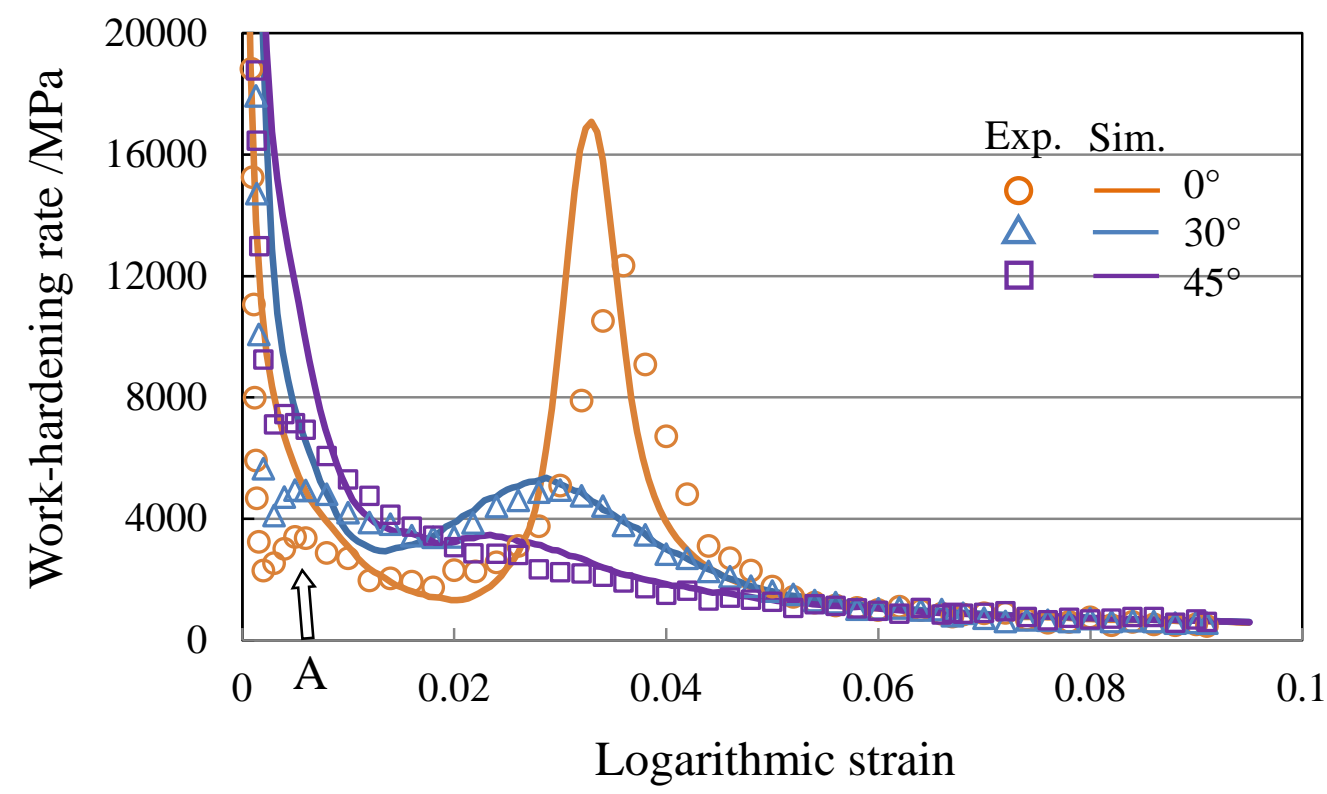

(b)

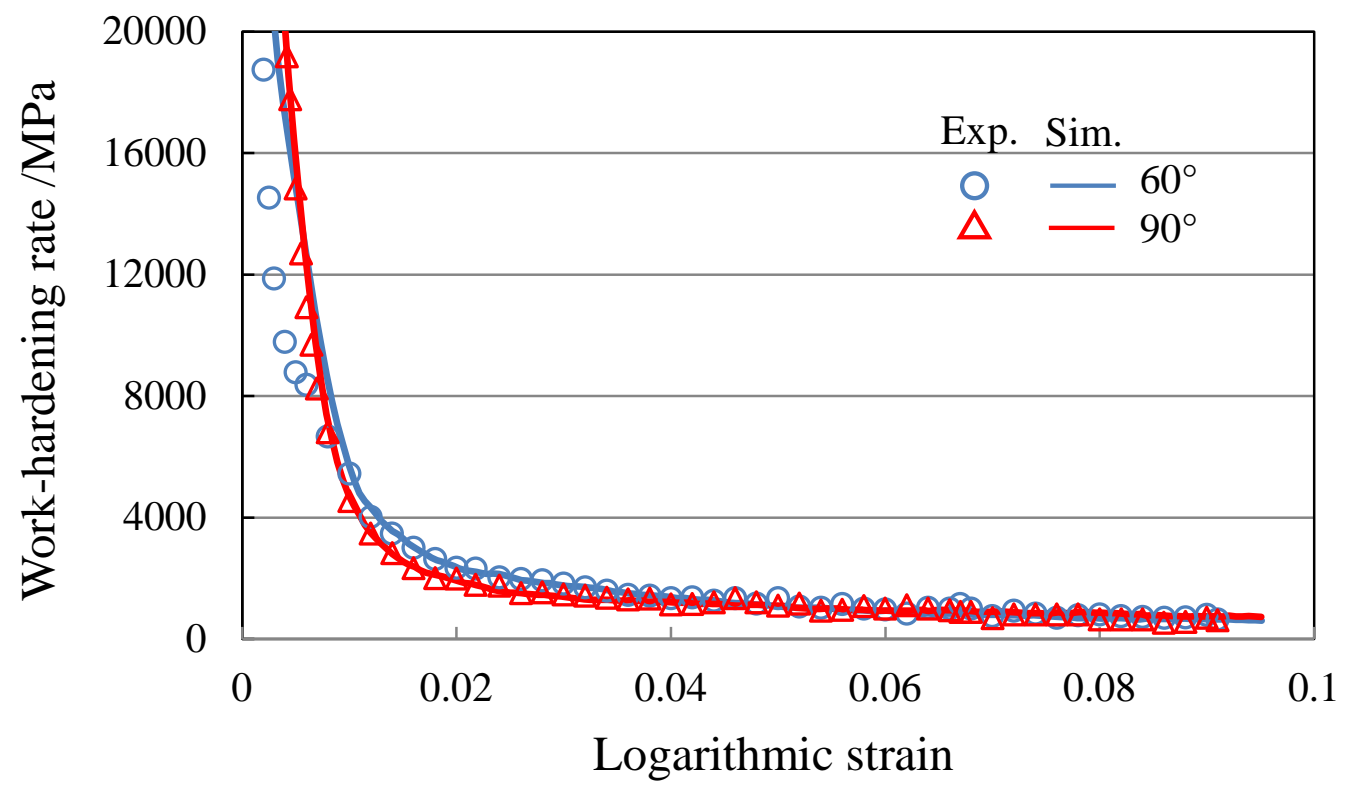

Fig. 9 Evolution of work-hardening rate during the second loading. The results of the $-3 \%$ prestrained sheet with (a) $\theta=0,30$ and $45^{\circ}$ and (b) $\theta=60$ and $90^{\circ}$, and the results of the $-6 \%$ prestrained sheet with (c) $\theta=0,30$ and $45^{\circ}$ and (d) $\theta=60$ and $90^{\circ}$. Symbols and solid lines are the experimental and simulation results, respectively. 
(c)

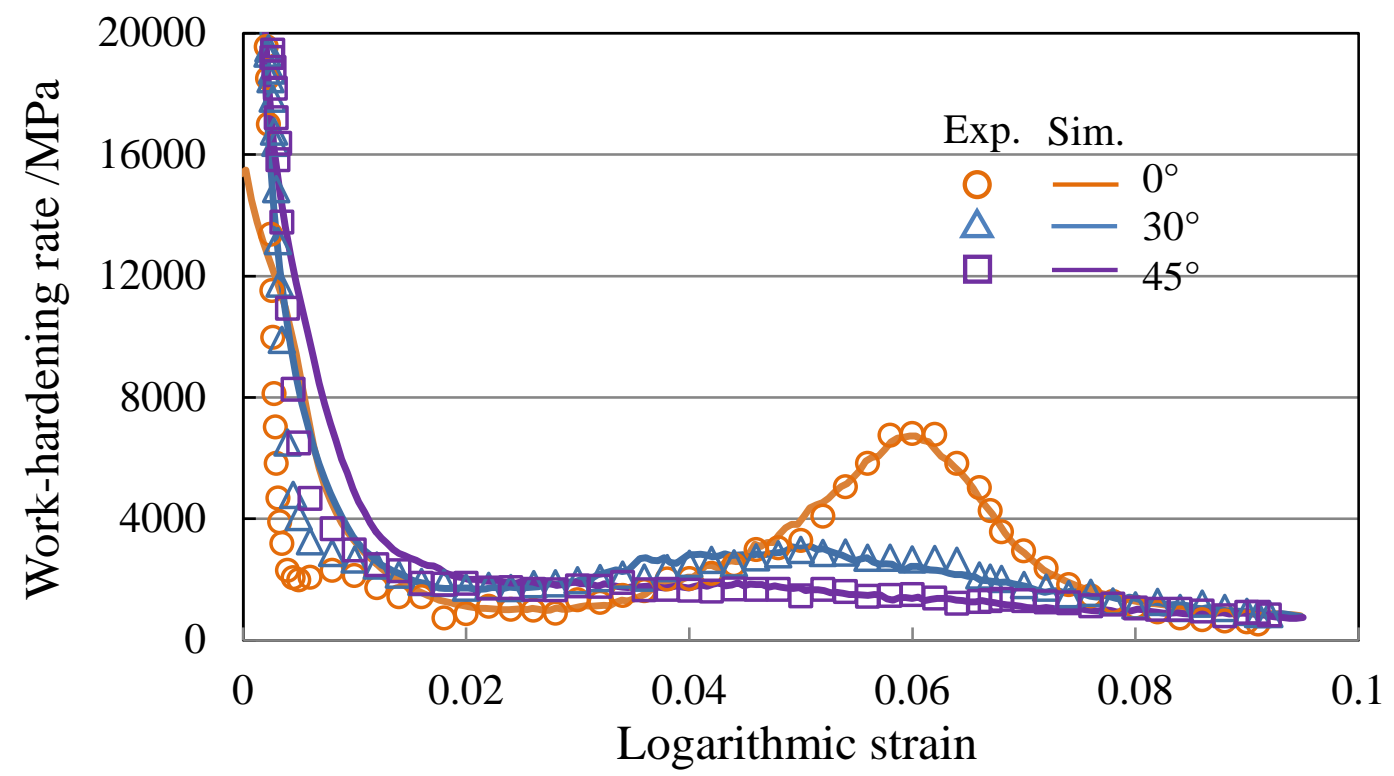

(d)

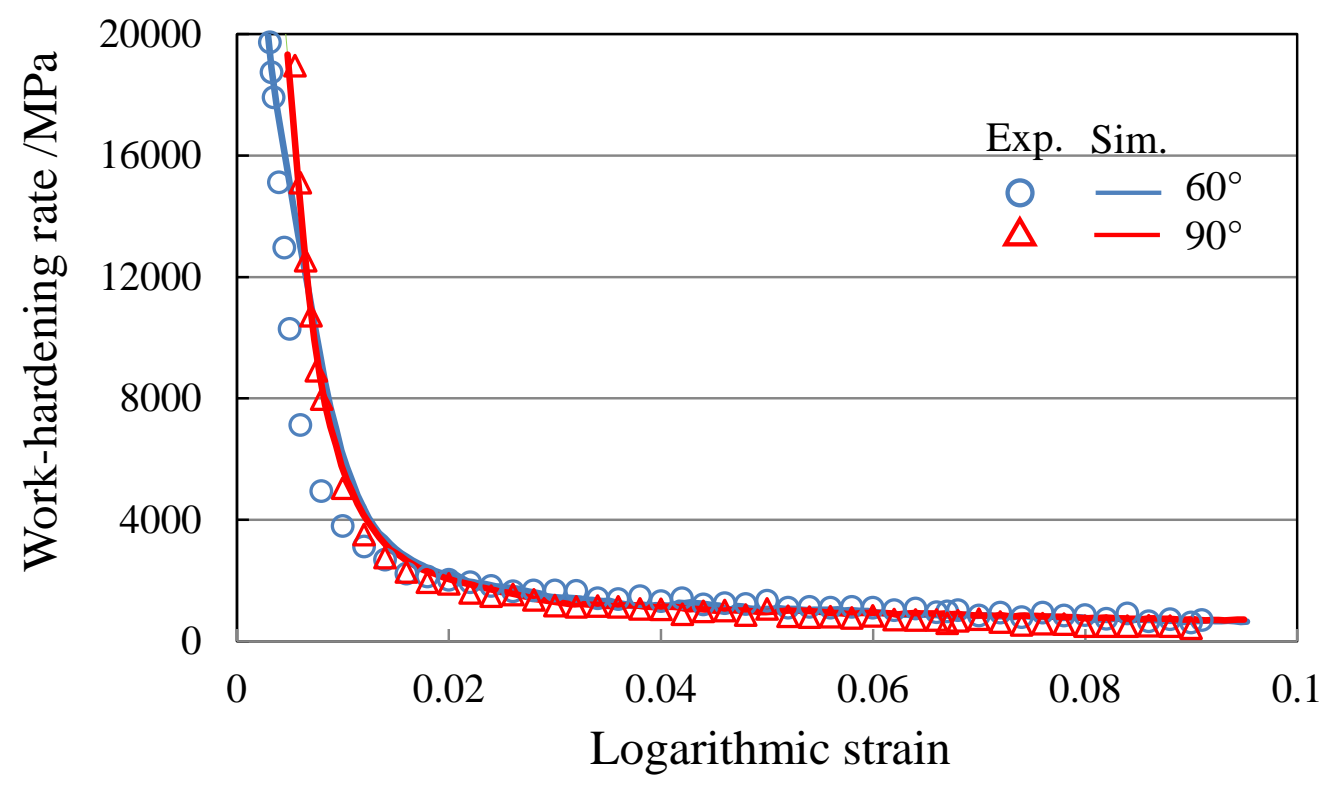

Fig. 9 Evolution of work-hardening rate during the second loading. The results of the $-3 \%$ prestrained sheet with (a) $\theta=0,30$ and $45^{\circ}$ and (b) $\theta=60$ and $90^{\circ}$, and the results of the $-6 \%$ prestrained sheet with (c) $\theta=0,30$ and $45^{\circ}$ and (d) $\theta=60$ and $90^{\circ}$. Symbols and solid lines are the experimental and simulation results, respectively. 


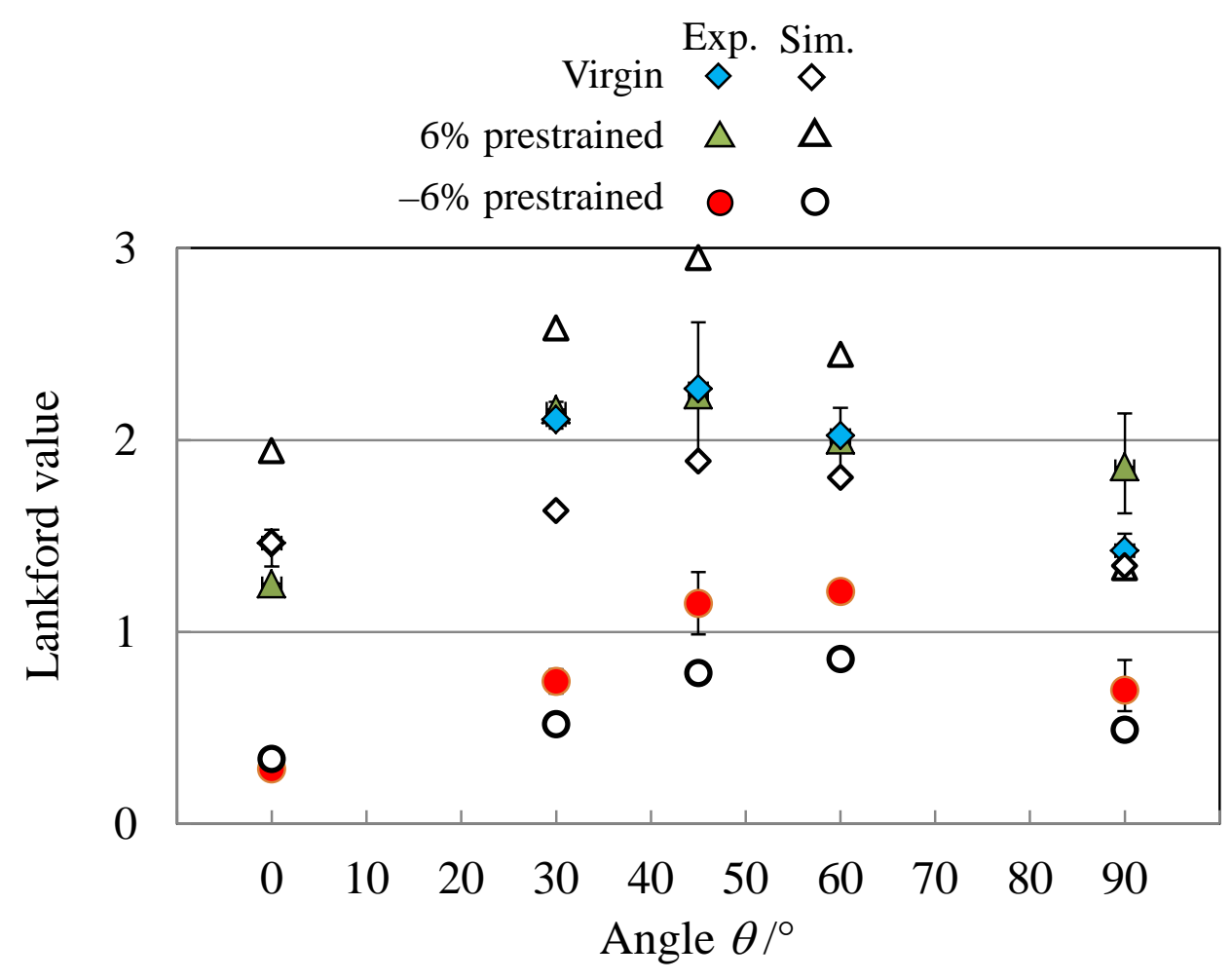

Fig. 10 Lankford values measured after the second loading was completed. 
(a)

Experiment $(\max =10.787)$
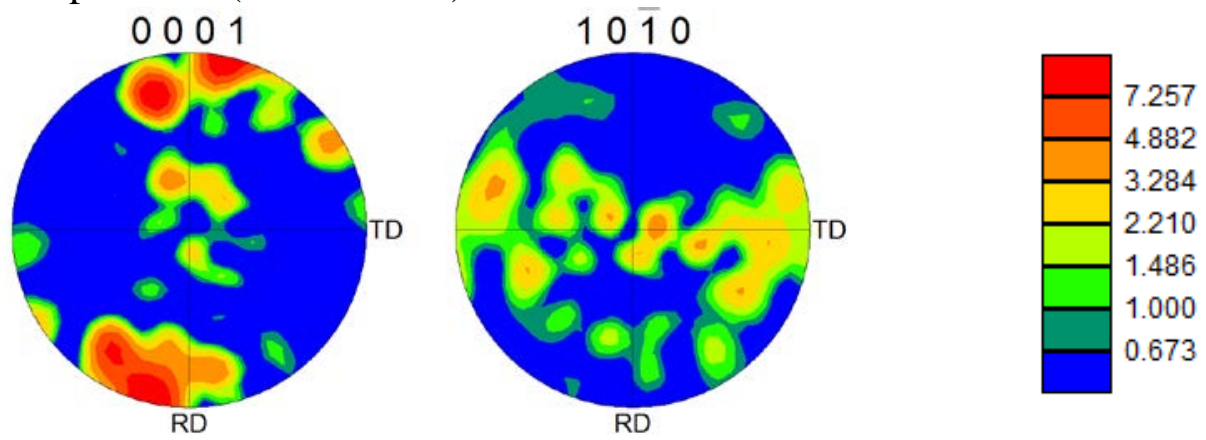

Simulation $(\max =6.424)$
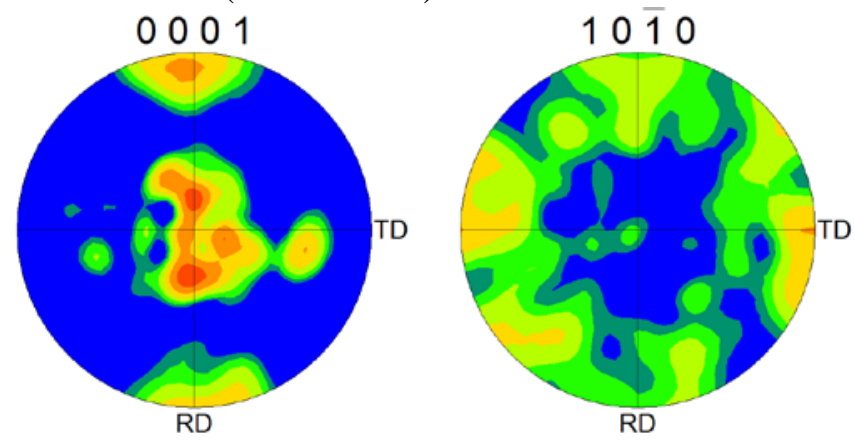

(b)

Experiment $(\max =12.636)$
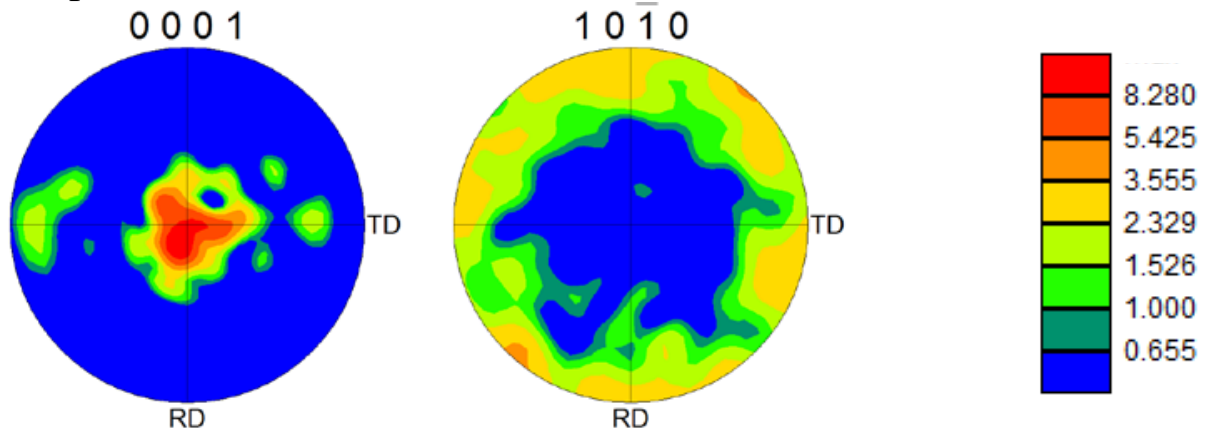

Simulation $(\max =8.918)$
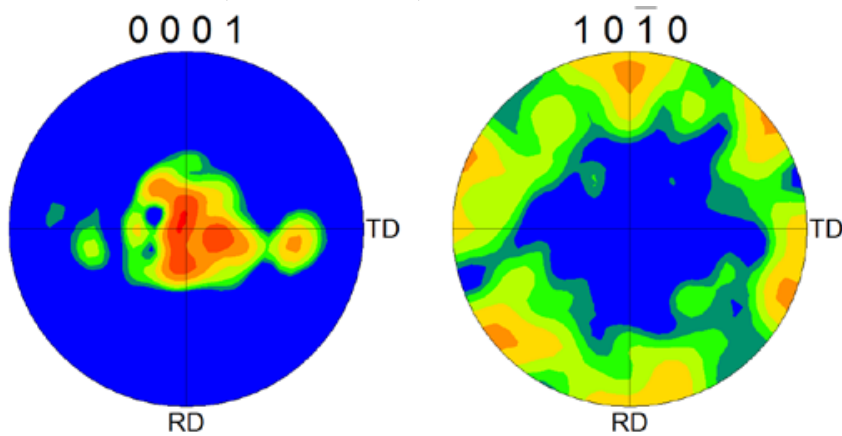
(c)

Experiment $(\max =7.704)$
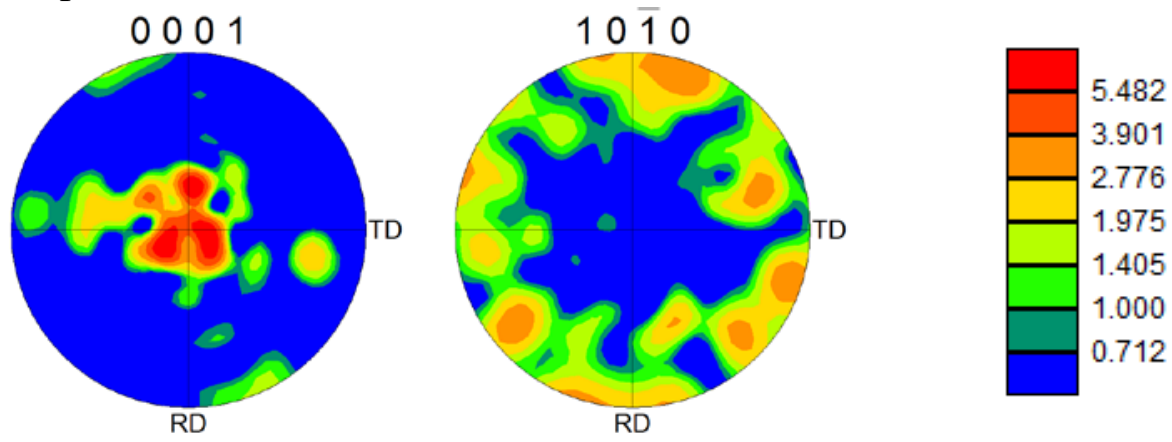

Simulation $(\max =7.335)$
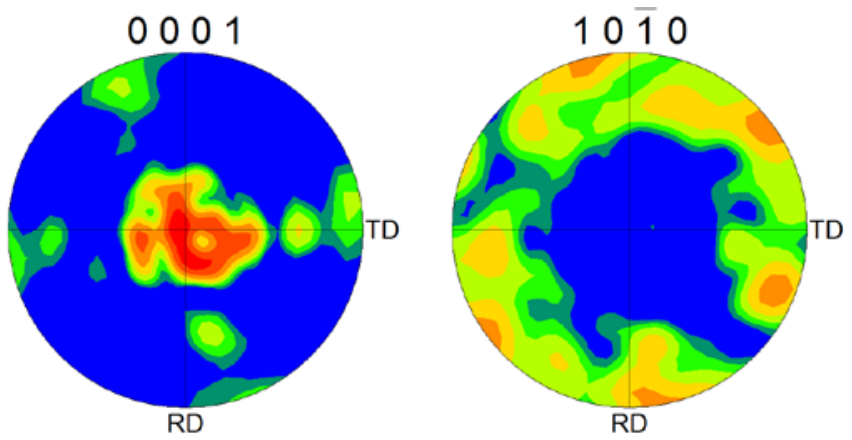

(d)

Experiment $(\max =7.520)$
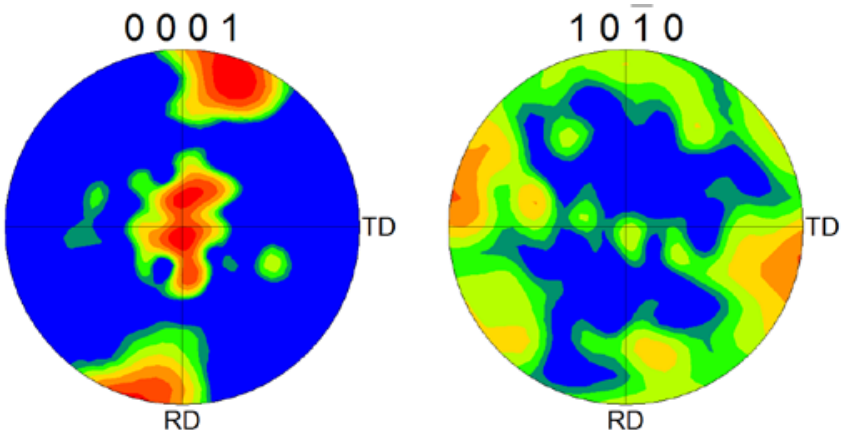

5.373

3.838

2.742

1.959

1.400

1.000

0.714

Simulation $(\max =7.335)$
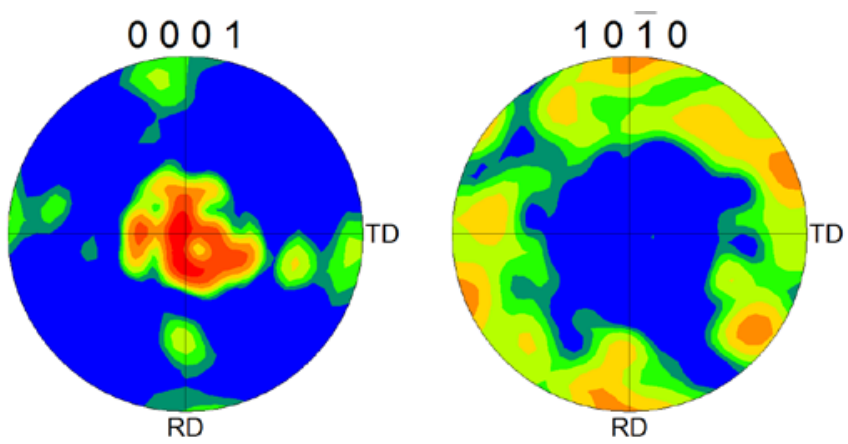
(e)

Experiment $(\max =7.934)$
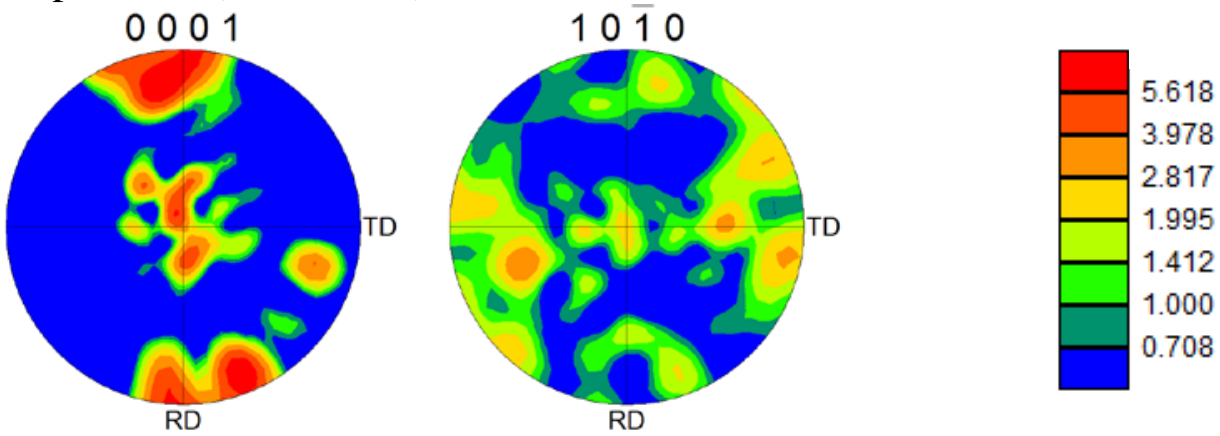

Simulation $(\max =6.269)$
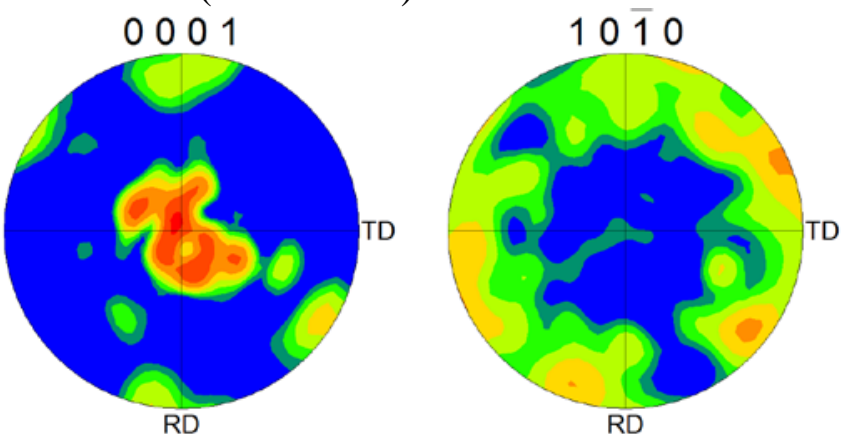

(f)

Experiment $(\max =11.824)$
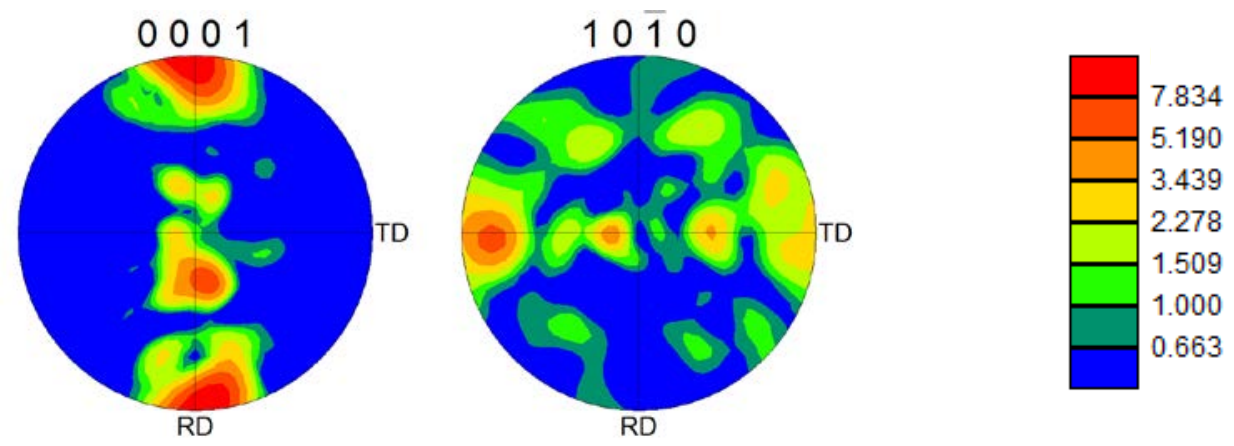

Simulation $(\max =6.737)$
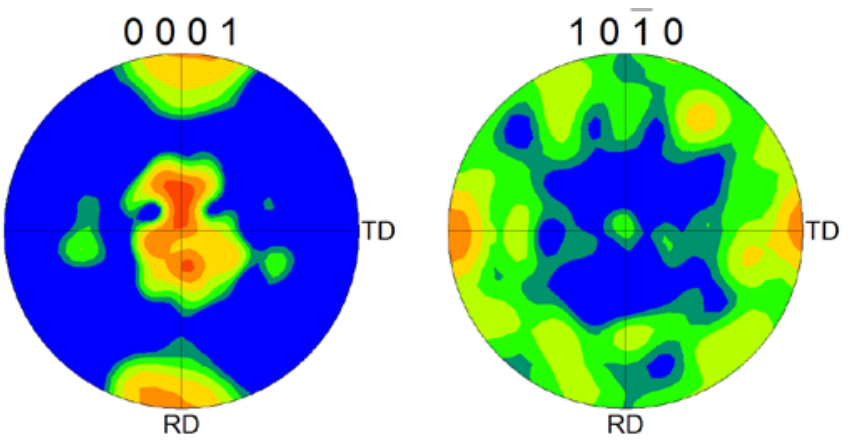

Fig. 11 Experimental and simulation results of pole figure for the $-6 \%$ prestrained sheet measured (a) before the second loading and after the second loading for $\theta$ of (b) 0 , (c) 30, (d) 45, (e) 60, and (f) $90^{\circ}$. 
(a)

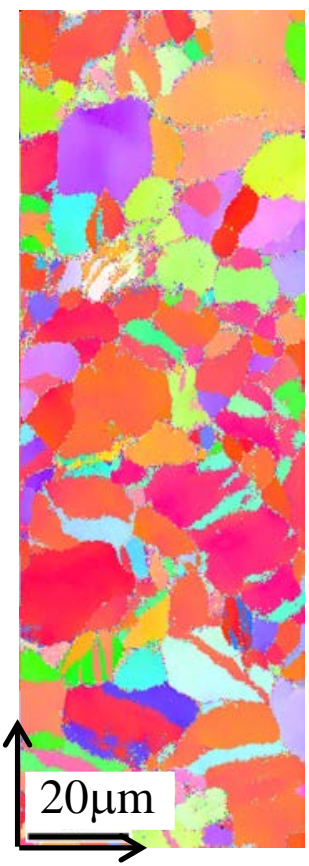

TD

(d)

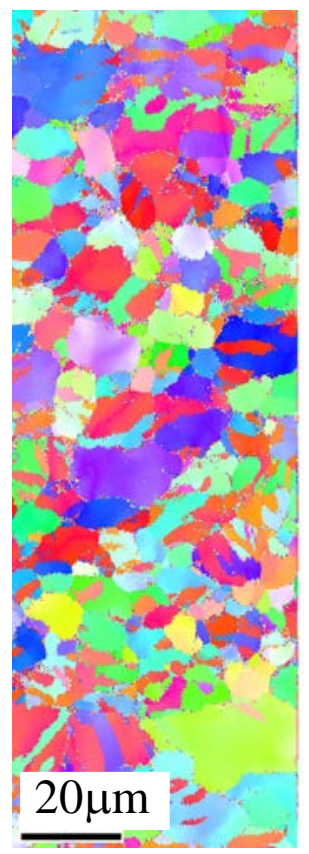

(b)

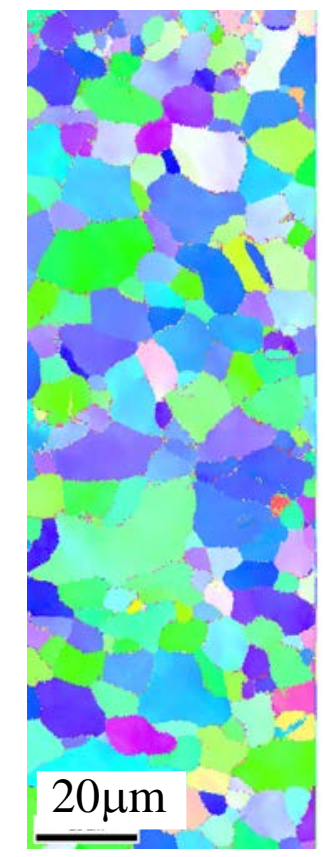

(e)

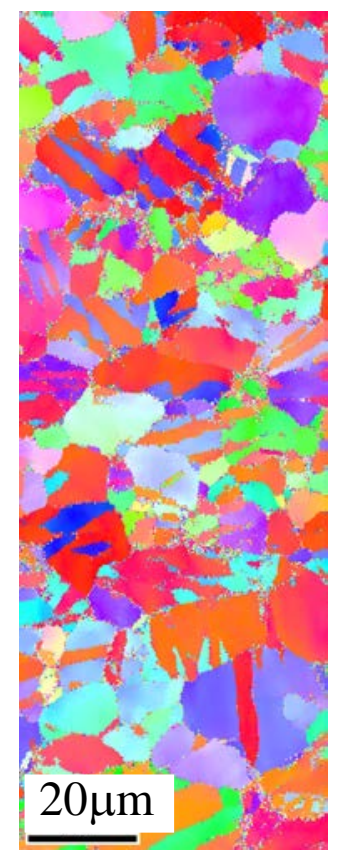

(c)

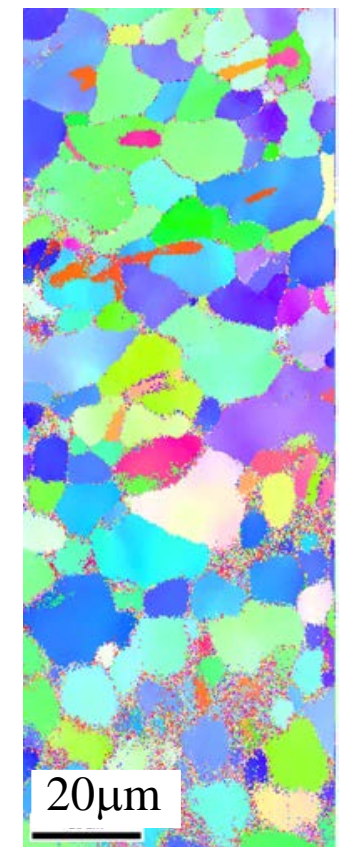

(f)

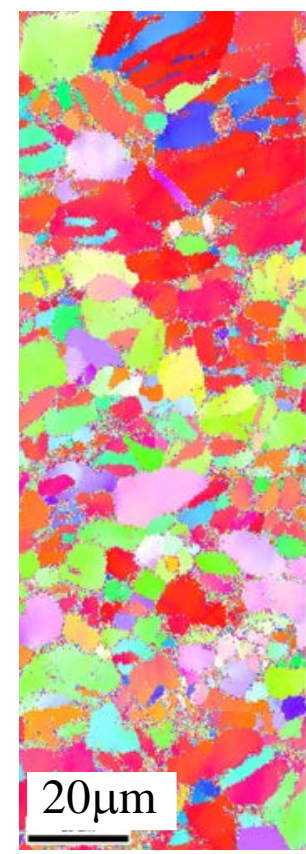

Fig. 12 Experimental results of inverse pole figure maps for the $-6 \%$ prestrained sheet measured (a) before the second loading and after the second loading for $\theta$ of (b) 0 , (c) 30, (d) 45, (e) 60, and (f) $90^{\circ}$. 


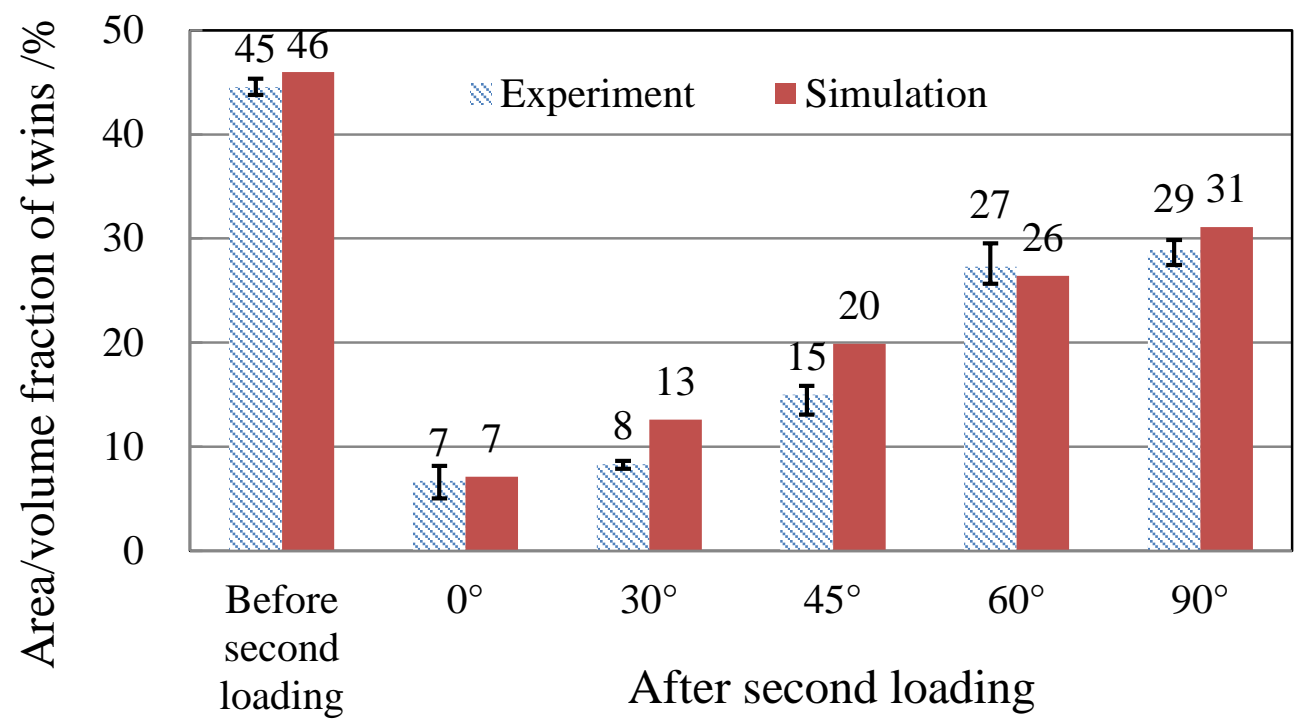

Fig. 13 Area/volume fractions of twins measured before and after the second loading in the $-6 \%$ prestrained sheet. 
(a)

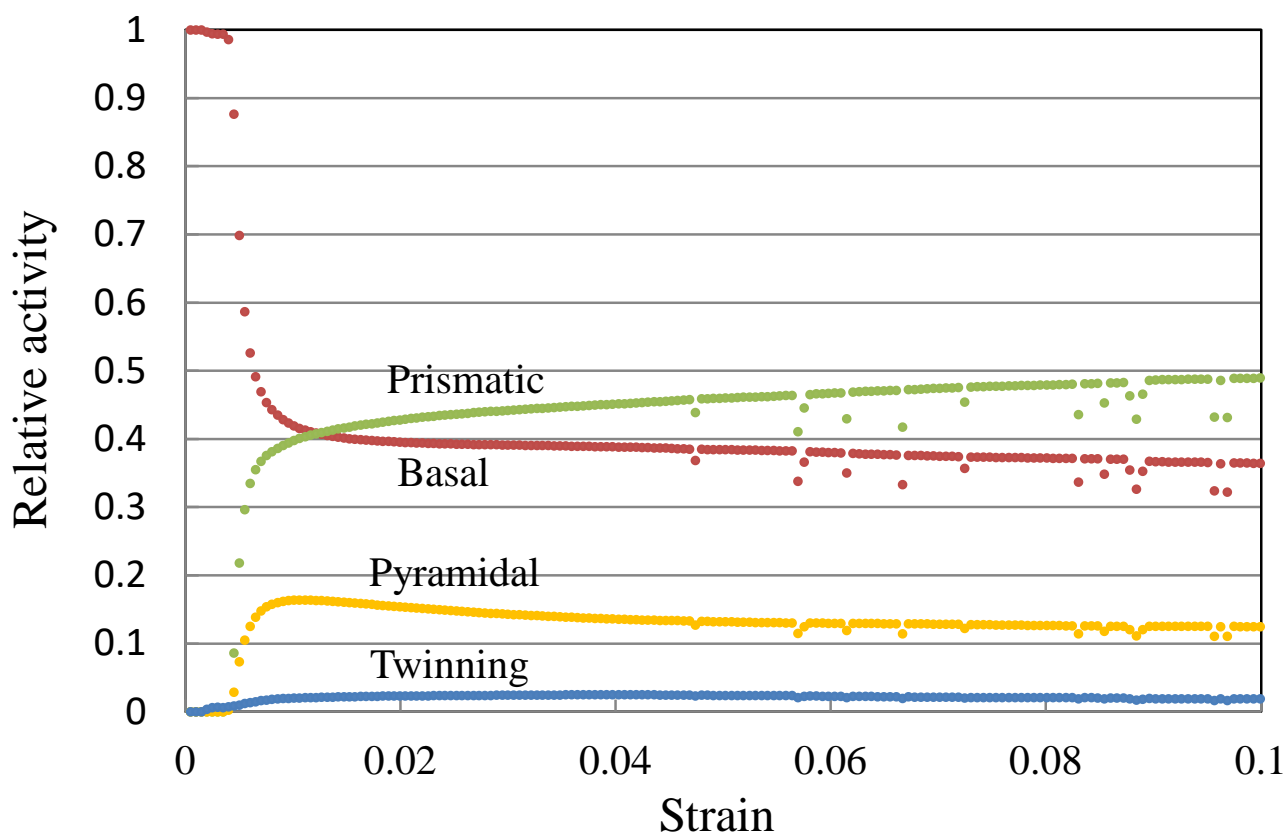

(b)

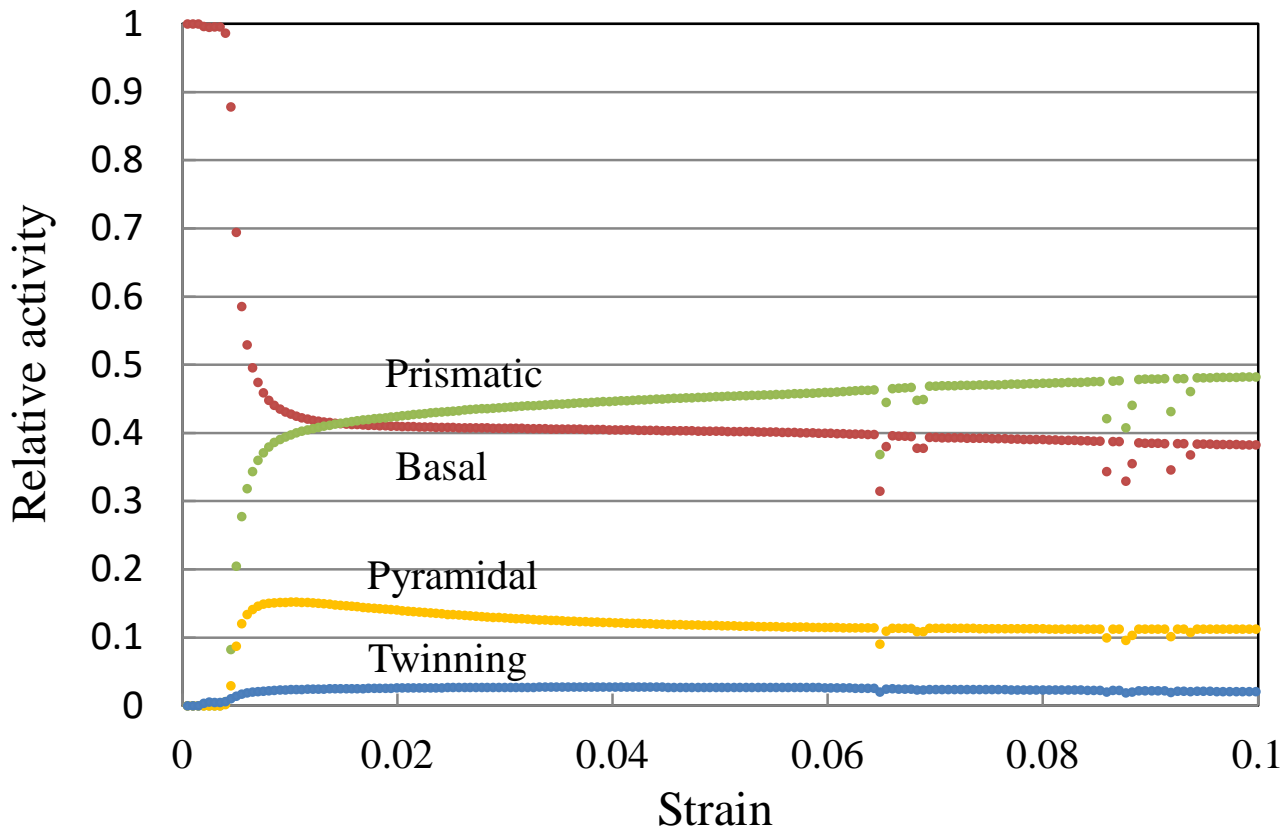

Fig. 14 Evolutions of relative activity in matrix region during monotonic tension in the virgin sheet at $\theta$ of (a) 0 , (b) 30 , (c) 45 , (d) 60 , and (e) $90^{\circ}$. 
(c)

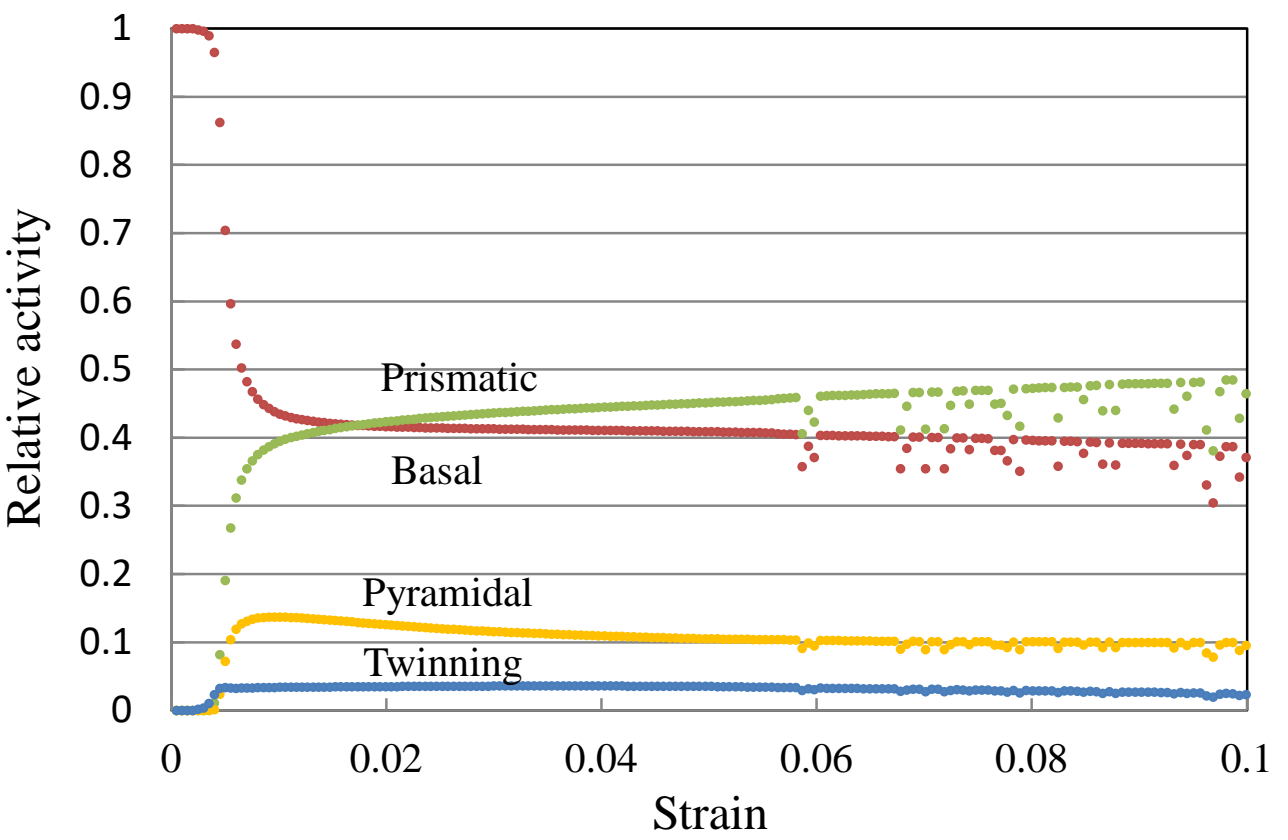

(d)

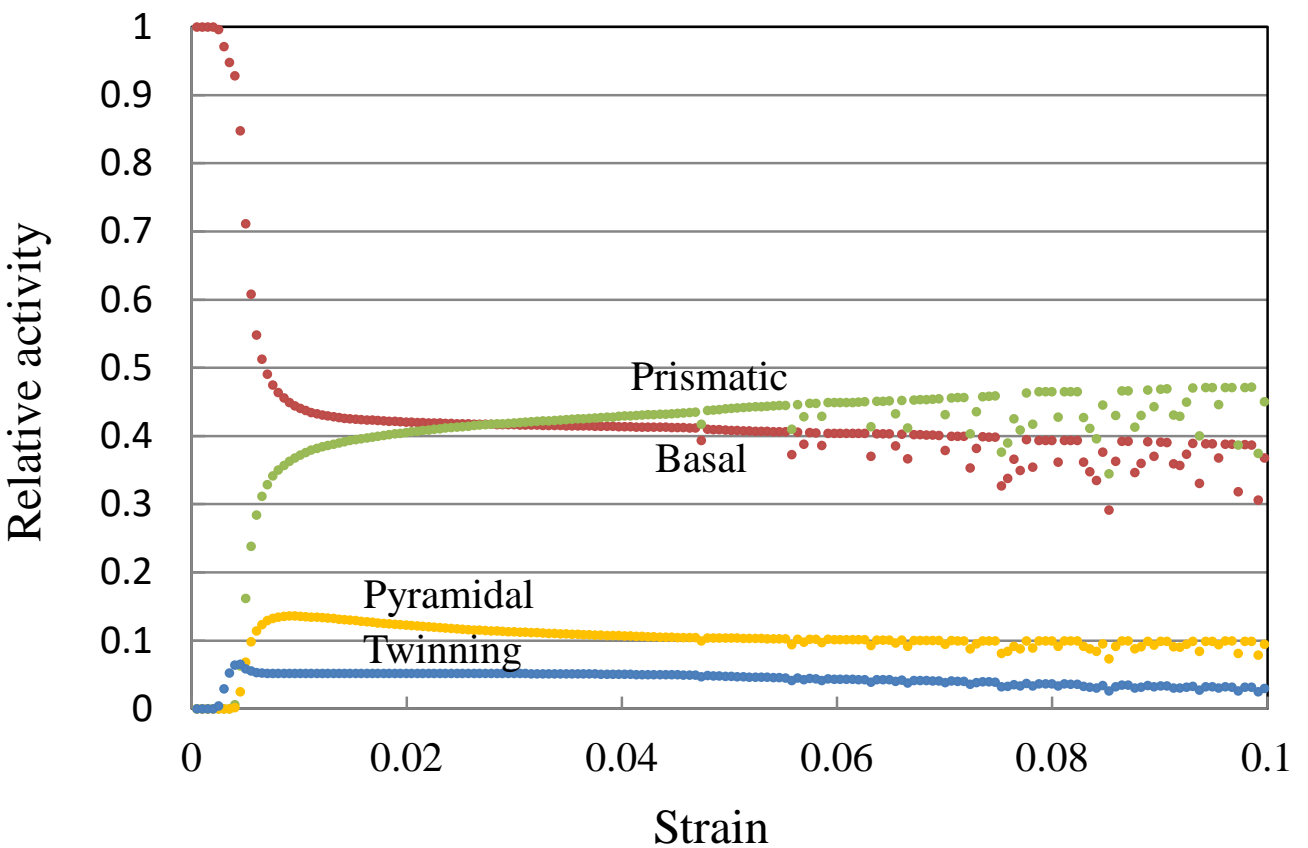

Fig. 14 Evolutions of relative activity in matrix region during monotonic tension in the virgin sheet at $\theta$ of (a) 0 , (b) 30 , (c) 45 , (d) 60 , and (e) $90^{\circ}$. 
(e)

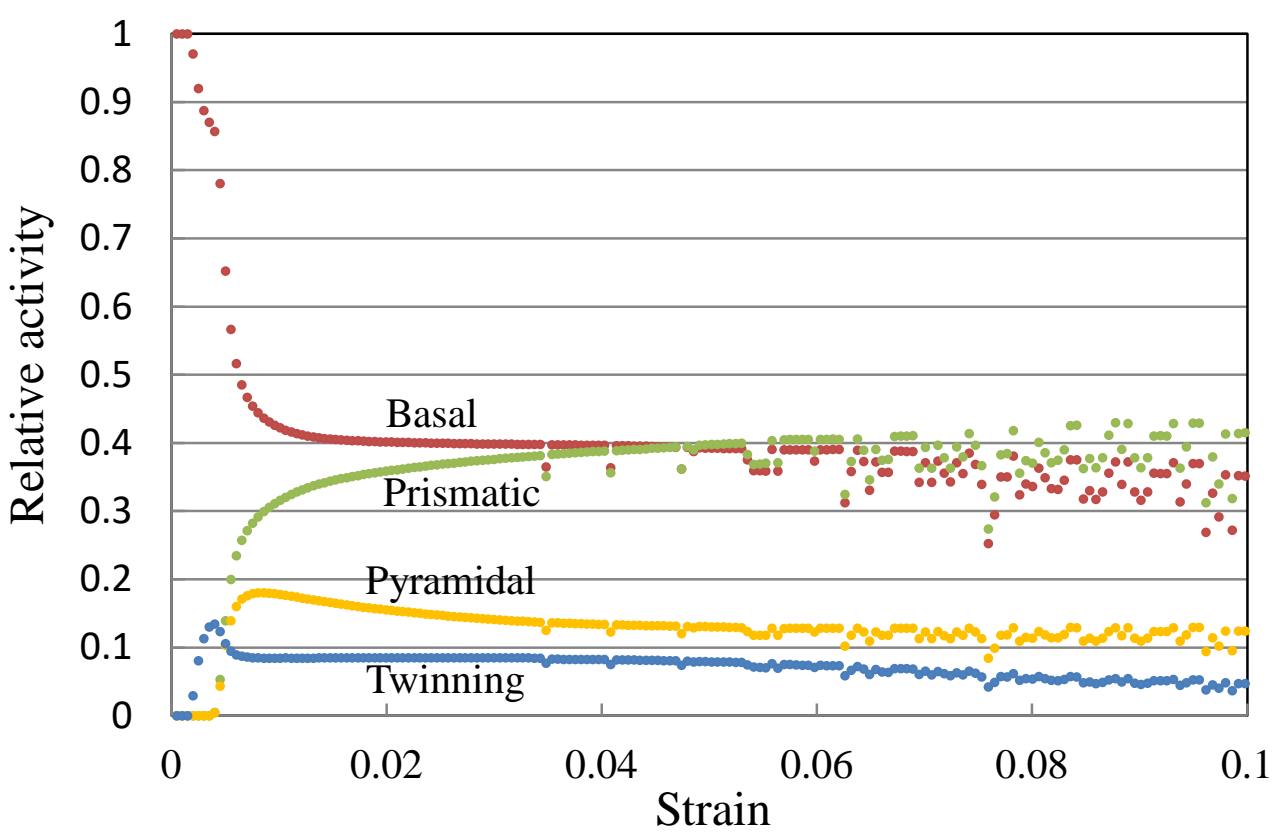

Fig. 14 Evolutions of relative activity in matrix region during monotonic tension in the virgin sheet at $\theta$ of (a) 0 , (b) 30, (c) 45, (d) 60, and (e) $90^{\circ}$. 
(a)

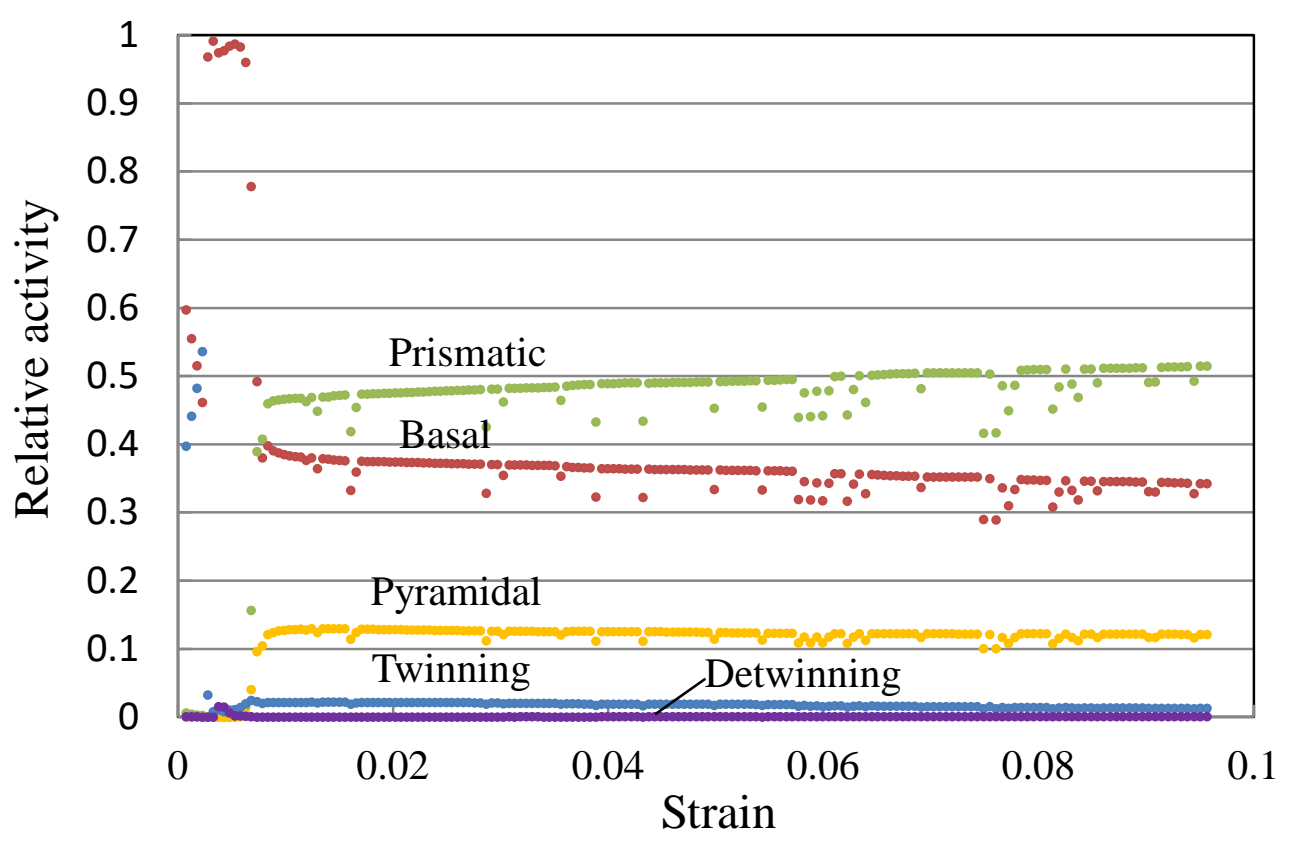

(b)

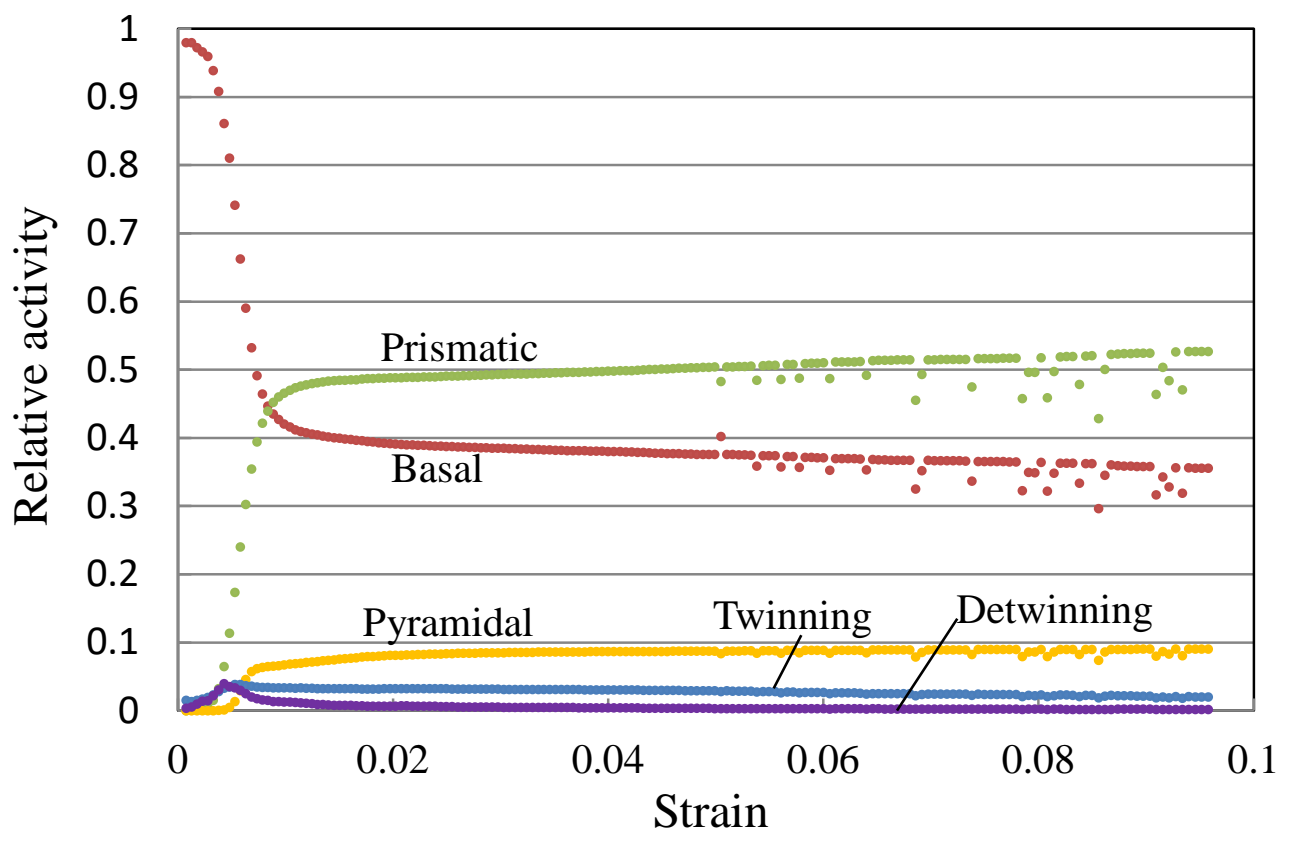

Fig. 15 Evolutions of relative activity in matrix region during the second loading in the $6 \%$ prestrained sheet at $\theta$ of (a) 0 , (b) 30, (c) 45 , (d) 60, and (e) $90^{\circ}$. 
(c)

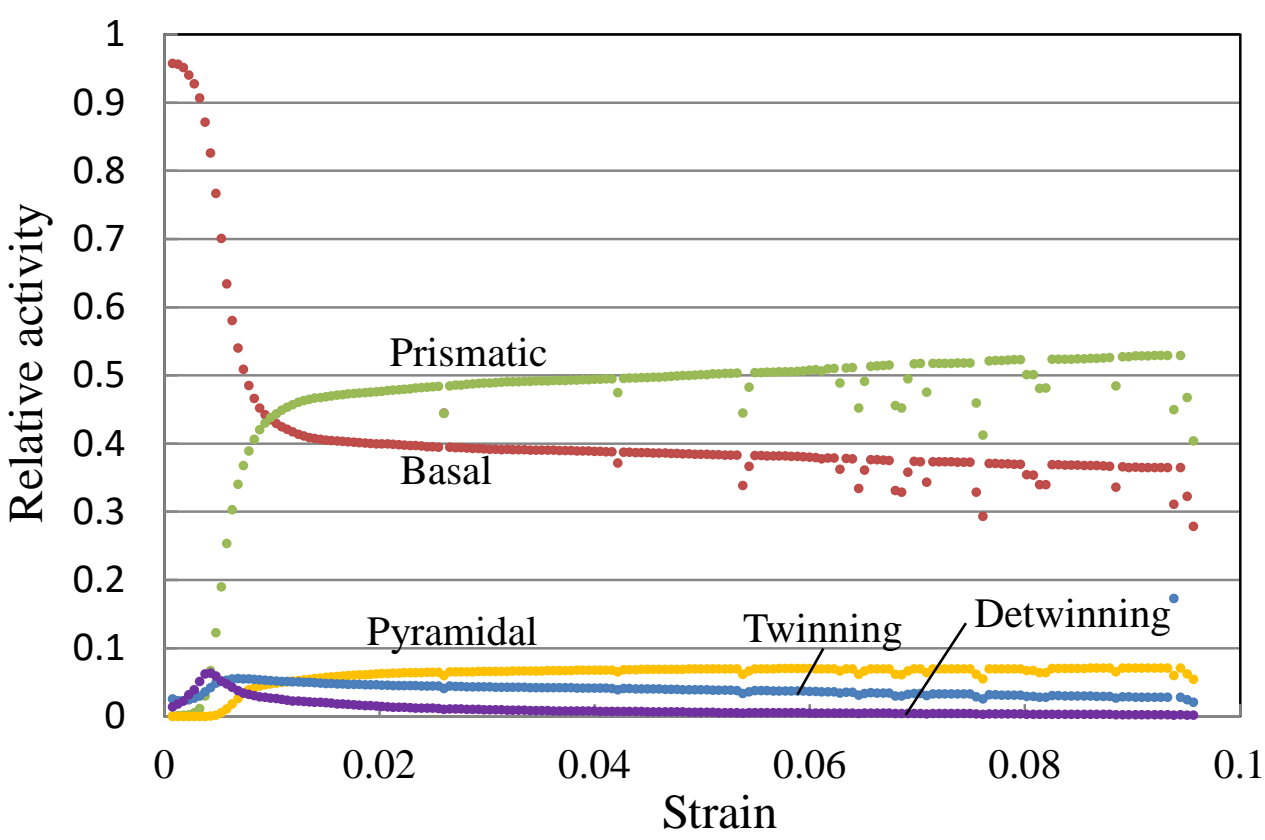

(d)

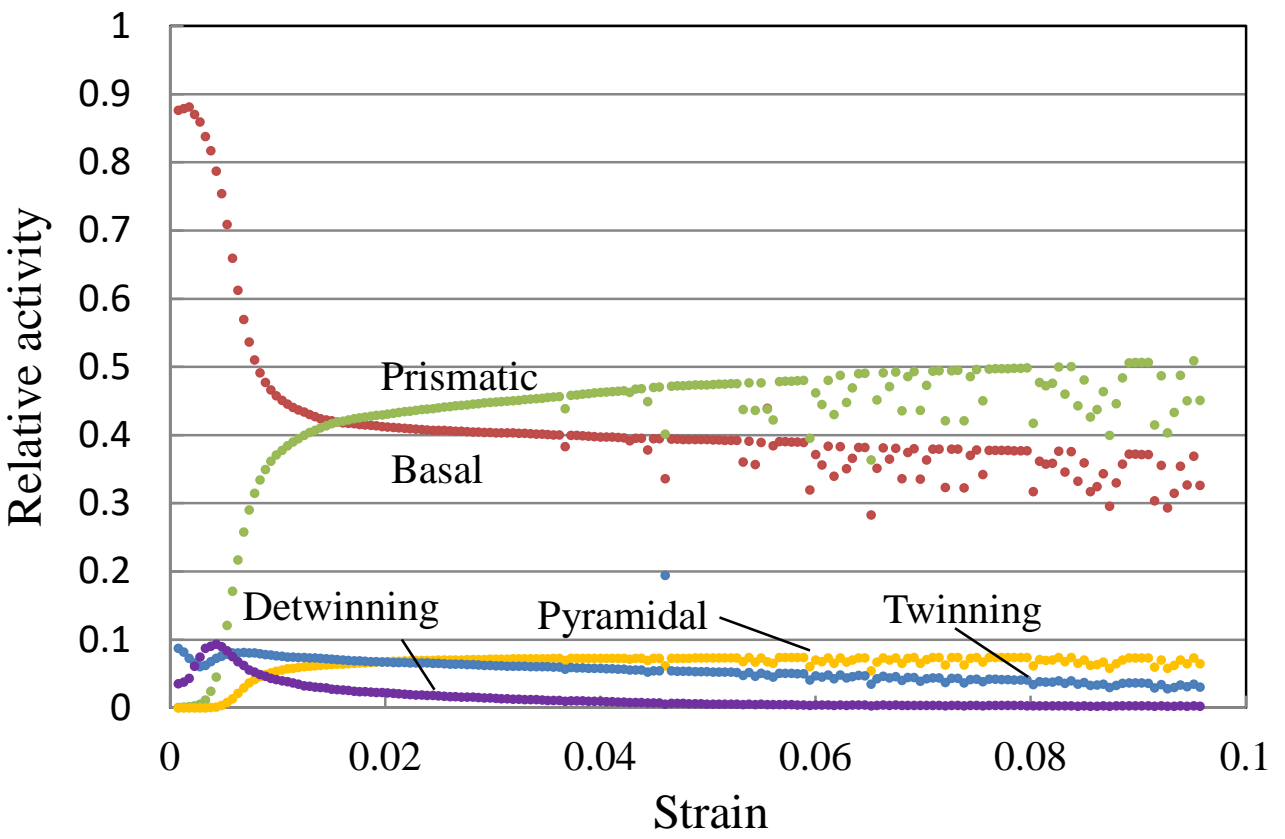

Fig. 15 Evolutions of relative activity in matrix region during the second loading in the $6 \%$ prestrained sheet at $\theta$ of (a) 0 , (b) 30, (c) 45 , (d) 60 , and (e) $90^{\circ}$. 
(e)

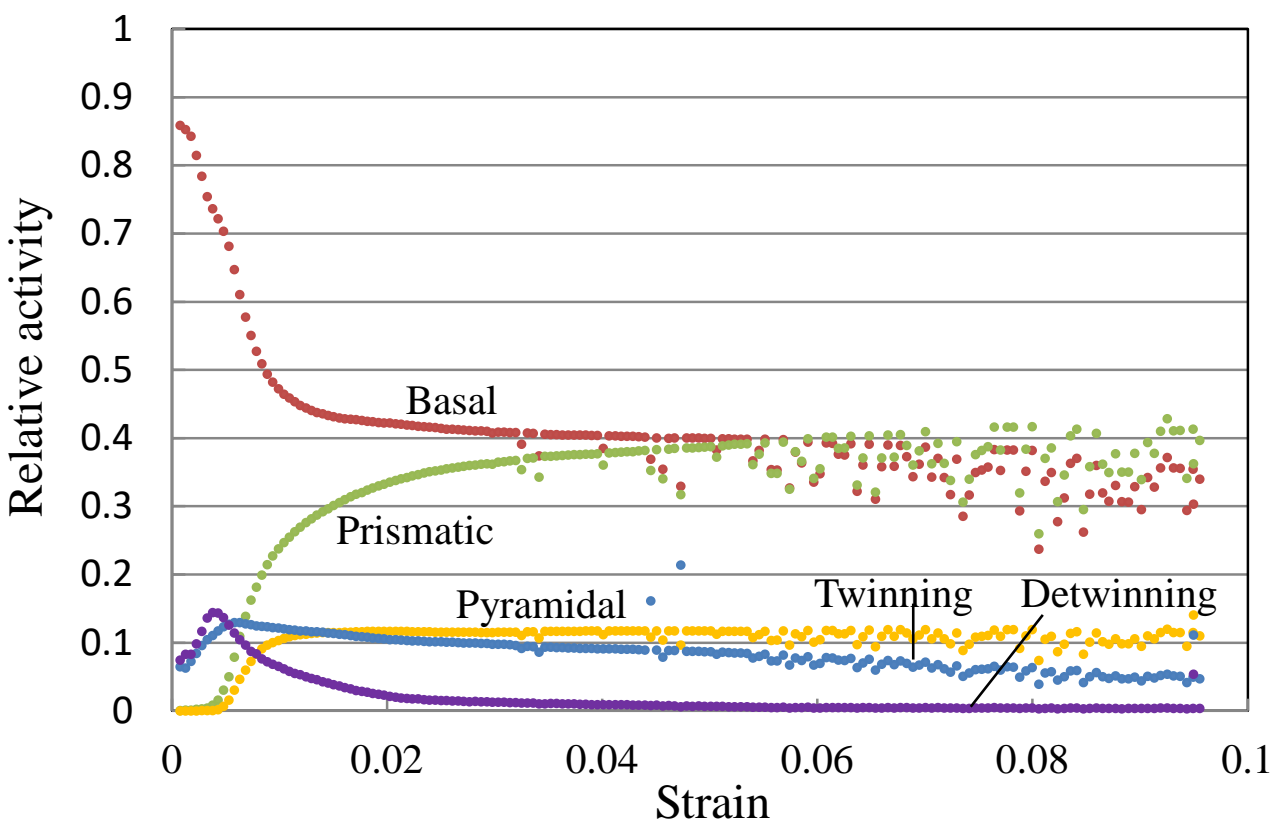

Fig. 15 Evolutions of relative activity in matrix region during the second loading in the $6 \%$ prestrained sheet at $\theta$ of (a) 0 , (b) 30, (c) 45, (d) 60, and (e) $90^{\circ}$. 


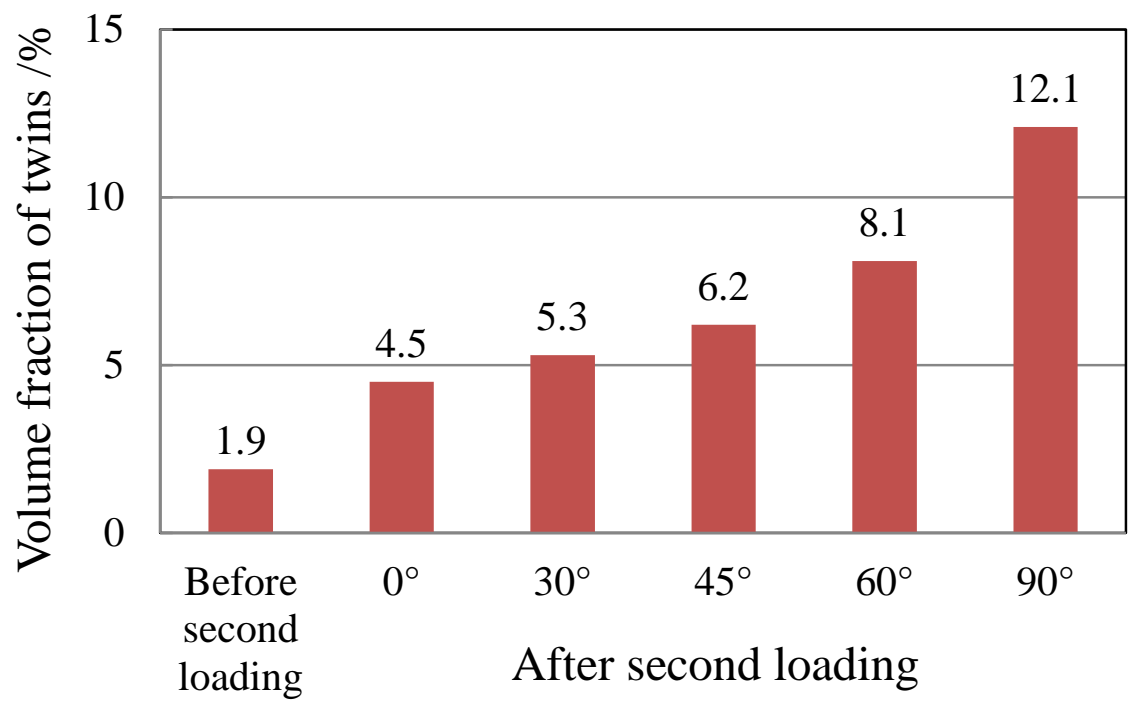

Fig. 16 Simulation results of volume fractions of twins measured before and after the second loading in the $6 \%$ prestrained sheet. 

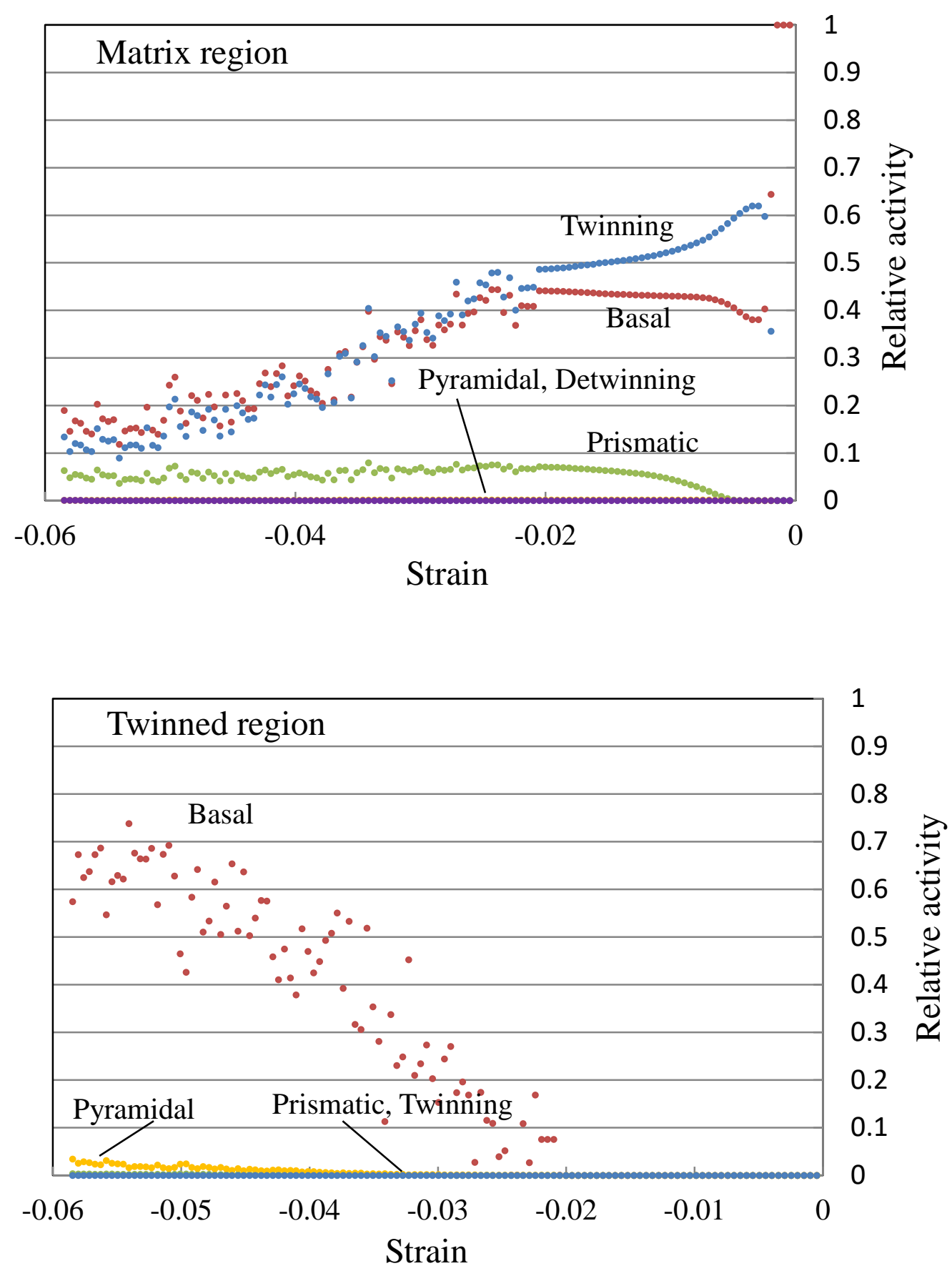

Fig. 17 Evolutions of relative activity during the first loading in the $-6 \%$ prestrained sheet. 
(a)
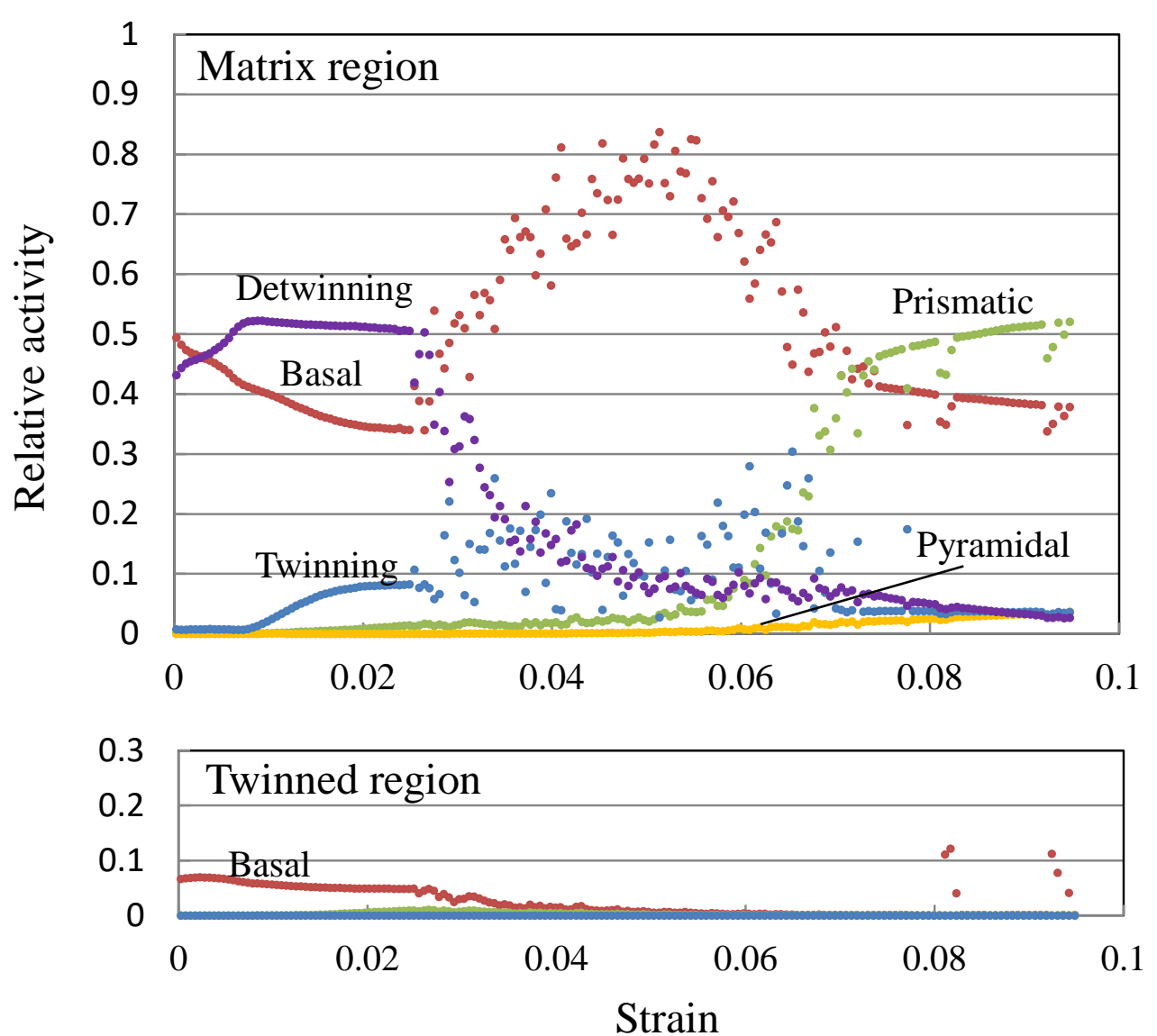

Fig. 18 Evolutions of relative activity during the second loading in the $-6 \%$ prestrained sheet at $\theta$ of (a) 0 , (b) 30, (c) 45, (d) 60, and (e) $90^{\circ}$. 
(b)
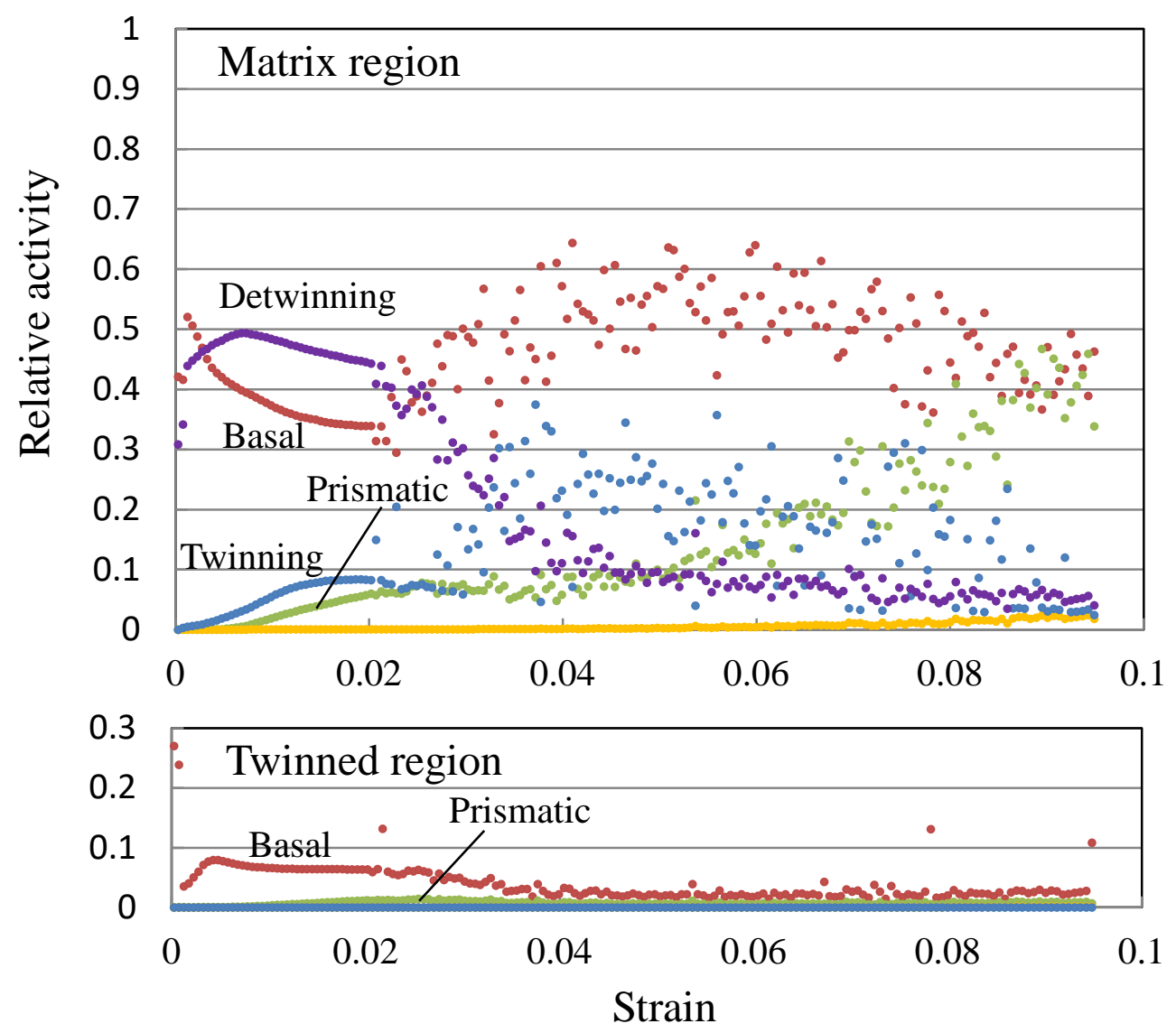

Fig. 18 Evolutions of relative activity during the second loading in the $-6 \%$ prestrained sheet at $\theta$ of (a) 0, (b) 30, (c) 45, (d) 60, and (e) $90^{\circ}$. 
(c)
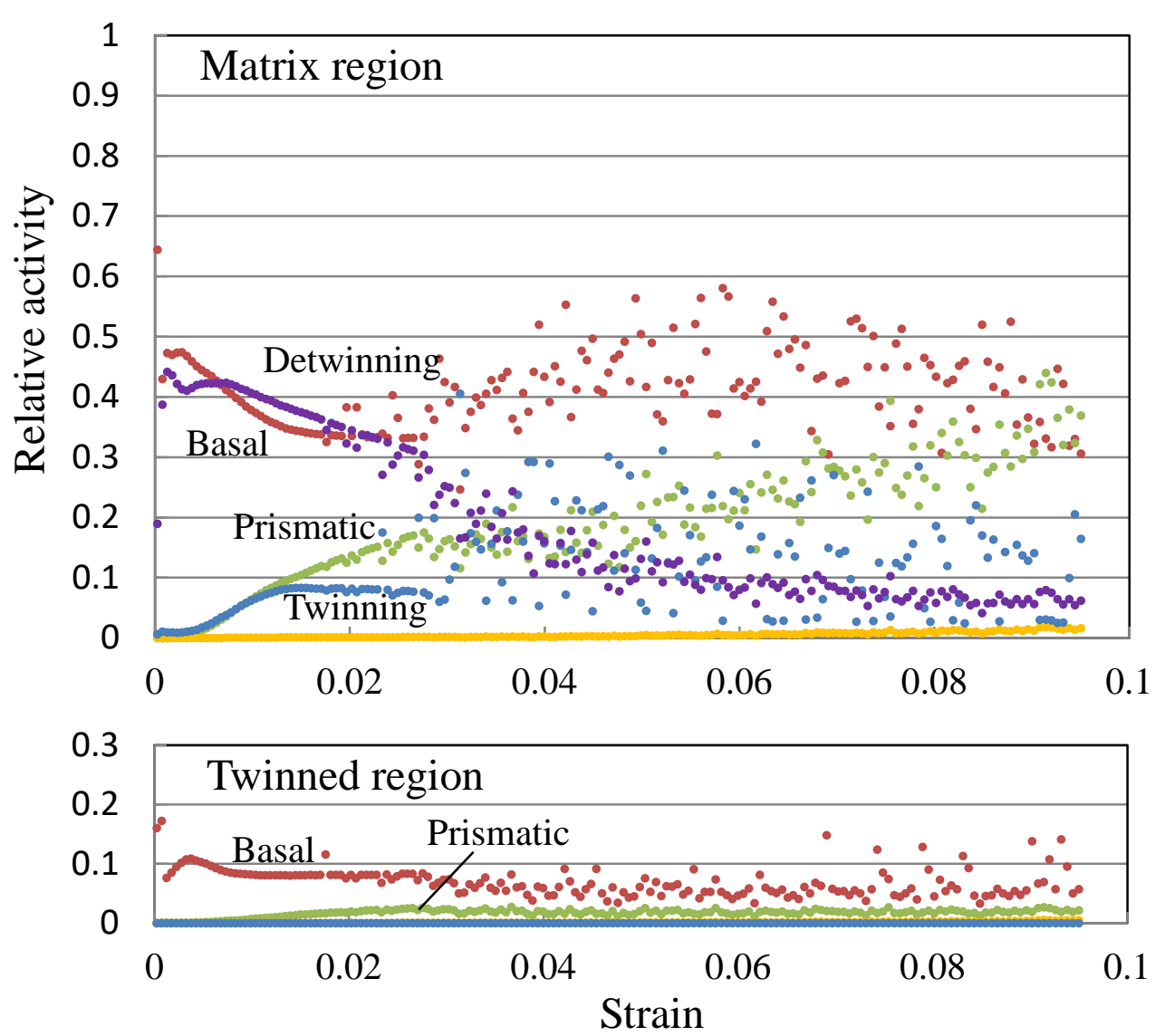

Fig. 18 Evolutions of relative activity during the second loading in the $-6 \%$ prestrained sheet at $\theta$ of (a) 0, (b) 30, (c) 45, (d) 60, and (e) $90^{\circ}$. 
(d)
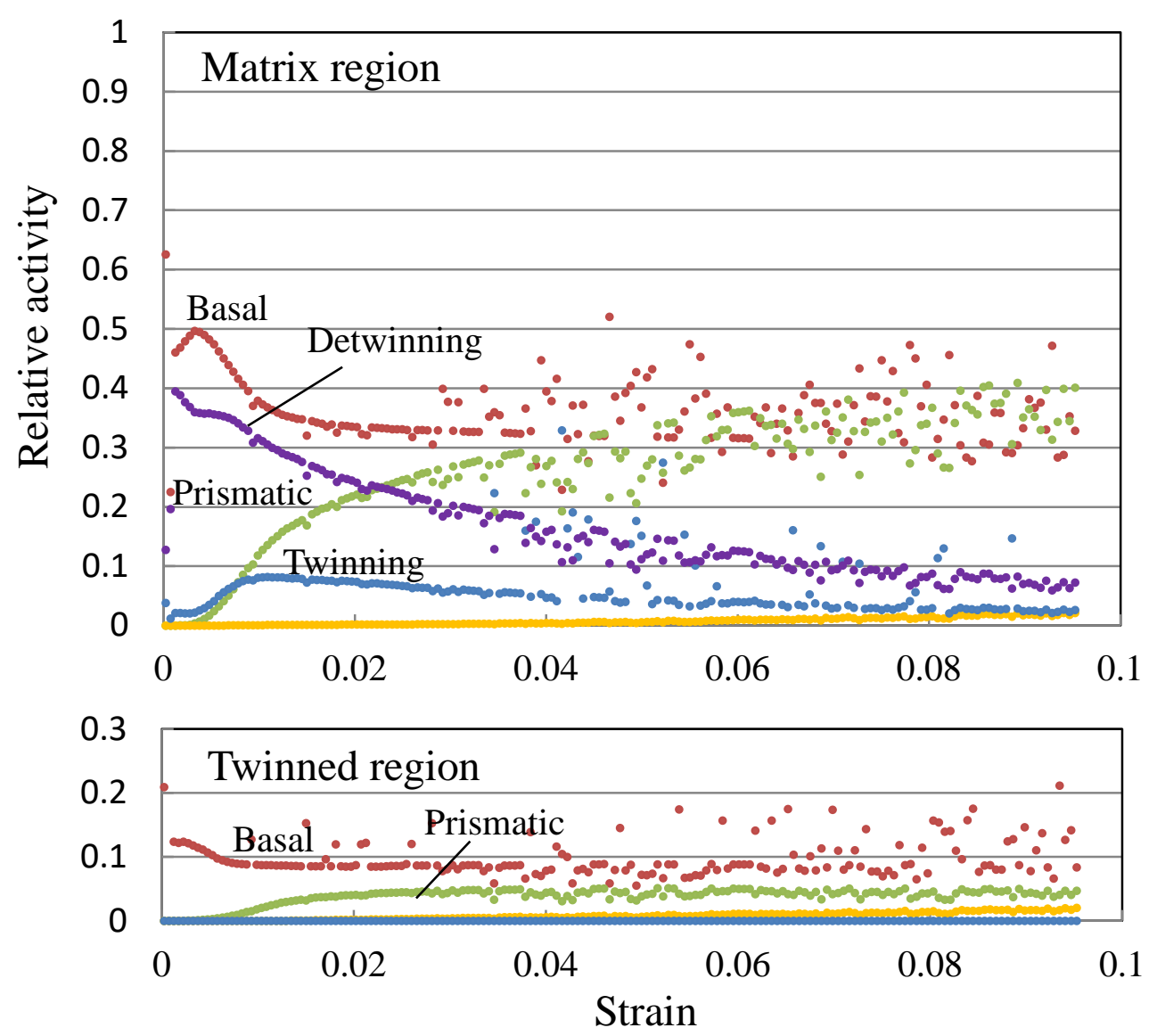

Fig. 18 Evolutions of relative activity during the second loading in the $-6 \%$ prestrained sheet at $\theta$ of (a) 0, (b) 30, (c) 45, (d) 60, and (e) $90^{\circ}$. 
(e)
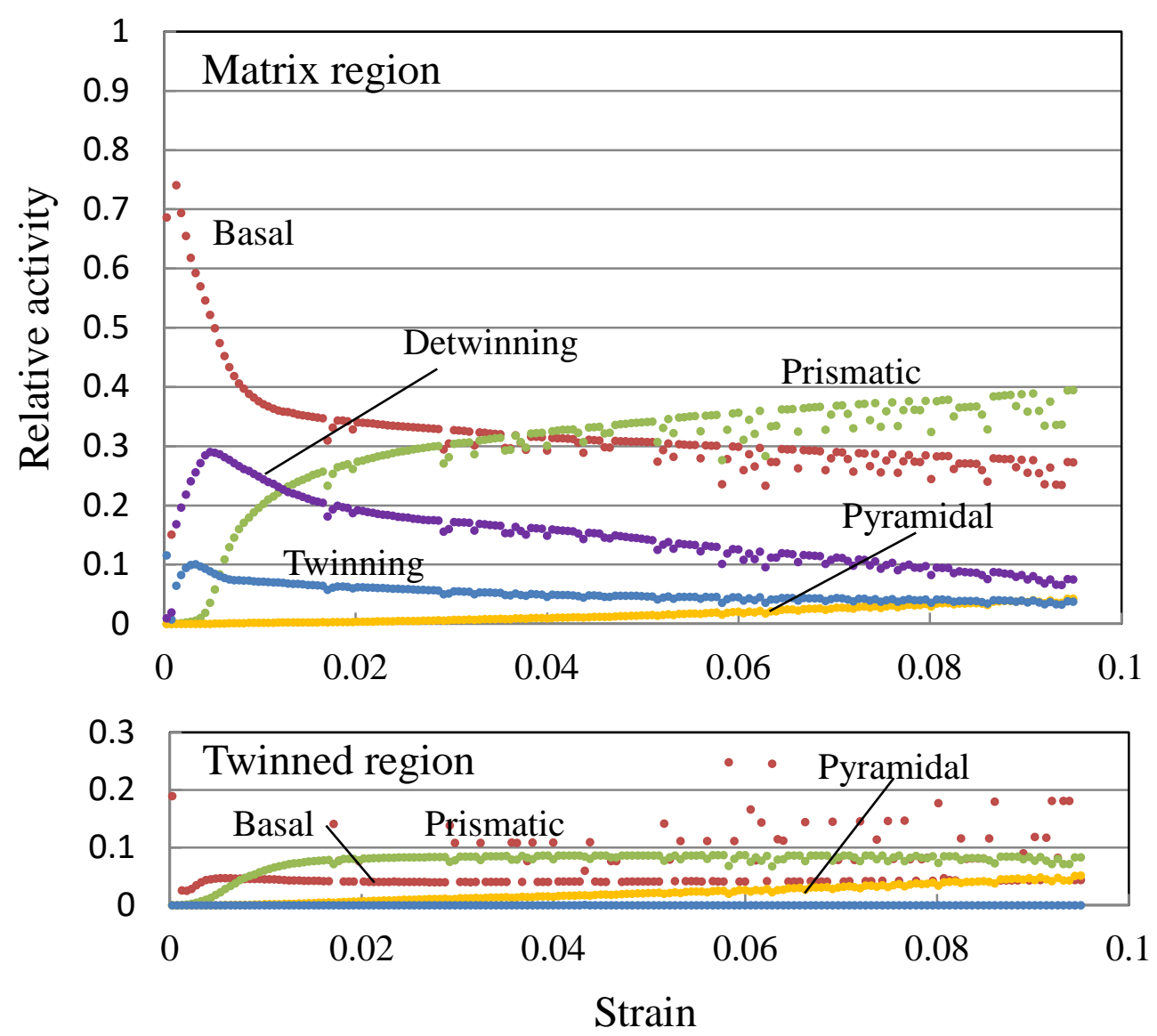

Fig. 18 Evolutions of relative activity during the second loading in the $-6 \%$ prestrained sheet at $\theta$ of (a) 0 , (b) 30, (c) 45, (d) 60, and (e) $90^{\circ}$. 


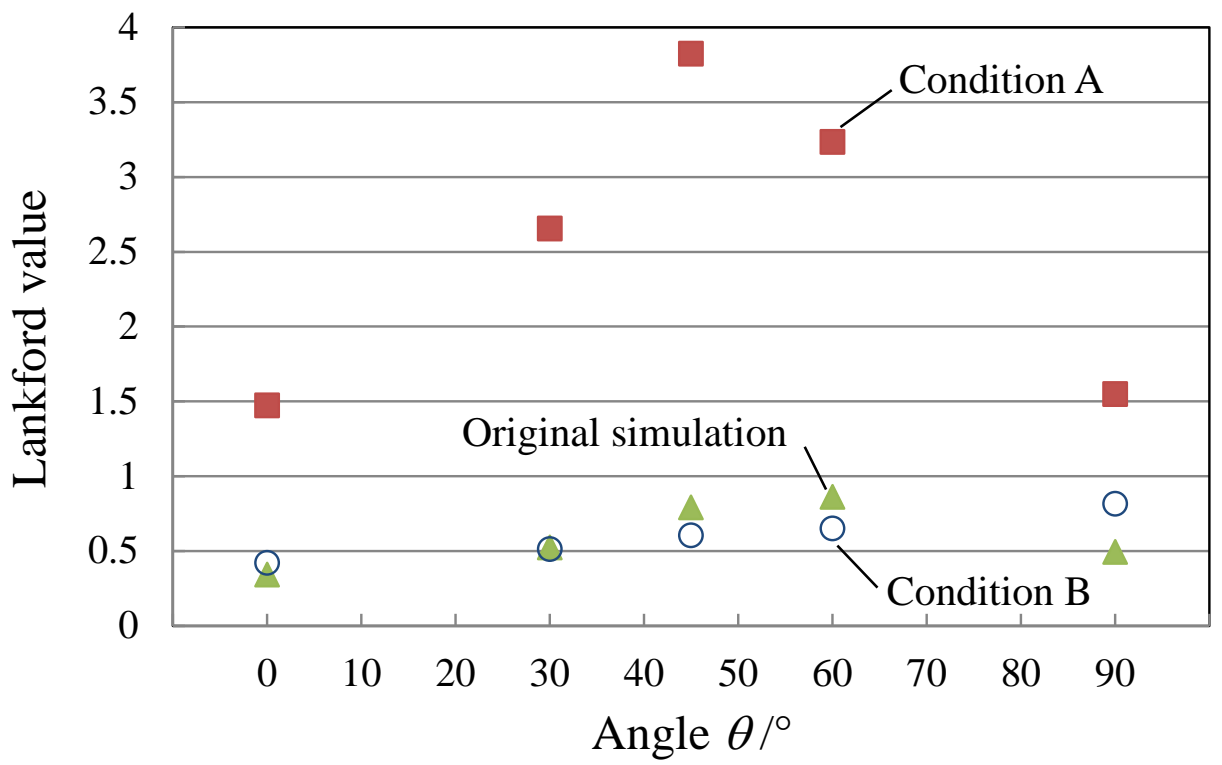

Fig. 19 Simulation results of Lankford value for the $-6 \%$ prestrained sheet with different numerical conditions. Condition A denotes the simulation where detwinning is not allowed, and condition B denotes the simulation where slip and twinning activities in the twinned region are not allowed. 


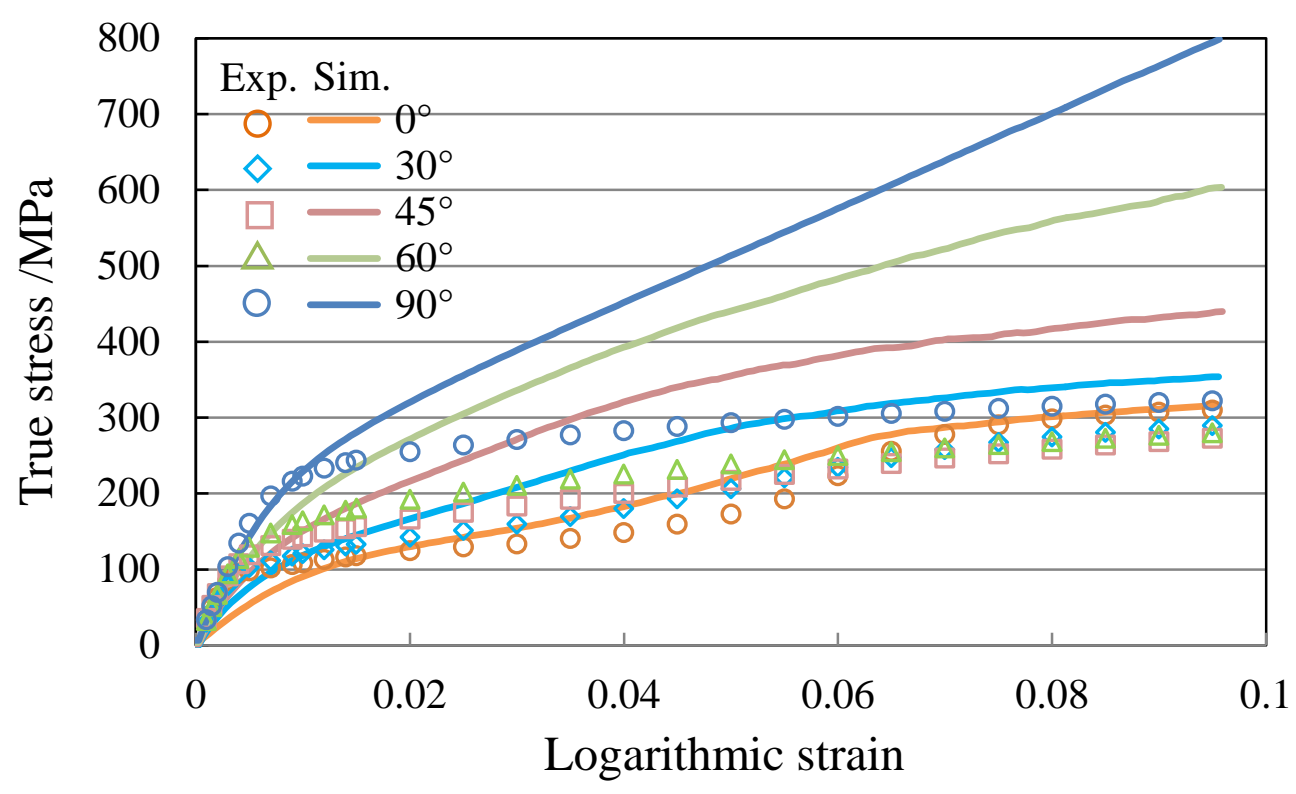

Fig. 20 Simulation results of stress-strain curve for the $-6 \%$ prestrained sheet where slip and twinning activities in the twinned region are not allowed. 